\title{
Study of the $B_{c}^{+} \rightarrow J / \psi D_{s}^{+}$and $B_{c}^{+} \rightarrow J / \psi D_{s}^{*+}$ decays with the ATLAS detector
}

\author{
ATLAS Collaboration ${ }^{\star}$ \\ CERN, 1211 Geneva 23, Switzerland
}

Received: 27 July 2015 / Accepted: 19 October 2015 / Published online: 5 January 2016

(C) CERN for the benefit of the ATLAS collaboration 2015. This article is published with open access at Springerlink.com

\begin{abstract}
The decays $B_{c}^{+} \rightarrow J / \psi D_{s}^{+}$and $B_{c}^{+} \rightarrow$ $J / \psi D_{s}^{*+}$ are studied with the ATLAS detector at the LHC using a dataset corresponding to integrated luminosities of 4.9 and $20.6 \mathrm{fb}^{-1}$ of $p p$ collisions collected at centre-ofmass energies $\sqrt{s}=7 \mathrm{TeV}$ and $8 \mathrm{TeV}$, respectively. Signal candidates are identified through $J / \psi \rightarrow \mu^{+} \mu^{-}$and $D_{s}^{(*)+} \rightarrow \phi \pi^{+}\left(\gamma / \pi^{0}\right)$ decays. With a two-dimensional likelihood fit involving the $B_{c}^{+}$reconstructed invariant mass and an angle between the $\mu^{+}$and $D_{s}^{+}$candidate momenta in the muon pair rest frame, the yields of $B_{c}^{+} \rightarrow J / \psi D_{s}^{+}$ and $B_{c}^{+} \rightarrow J / \psi D_{s}^{*+}$, and the transverse polarisation fraction in $B_{c}^{+} \rightarrow J / \psi D_{s}^{*+}$ decay are measured. The transverse polarisation fraction is determined to be $\Gamma_{ \pm \pm}\left(B_{c}^{+} \rightarrow\right.$ $\left.J / \psi D_{s}^{*+}\right) / \Gamma\left(B_{c}^{+} \rightarrow J / \psi D_{s}^{*+}\right)=0.38 \pm 0.23 \pm 0.07$, and the derived ratio of the branching fractions of the two modes is $\mathcal{B}_{B_{c}^{+} \rightarrow J / \psi D_{s}^{*+}} / \mathcal{B}_{B_{c}^{+} \rightarrow J / \psi D_{s}^{+}}=2.8_{-0.8}^{+1.2} \pm 0.3$, where the first error is statistical and the second is systematic. Finally, a sample of $B_{c}^{+} \rightarrow J / \psi \pi^{+}$decays is used to derive the ratios of branching fractions $\mathcal{B}_{B_{c}^{+} \rightarrow J / \psi D_{s}^{+}} / \mathcal{B}_{B_{c}^{+} \rightarrow J / \psi \pi^{+}}=$ $3.8 \pm 1.1 \pm 0.4 \pm 0.2$ and $\mathcal{B}_{B_{c}^{+} \rightarrow J / \psi D_{s}^{*+}} / \mathcal{B}_{B_{c}^{+} \rightarrow J / \psi \pi^{+}}=$ $10.4 \pm 3.1 \pm 1.5 \pm 0.6$, where the third error corresponds to the uncertainty of the branching fraction of $D_{S}^{+} \rightarrow$ $\phi\left(K^{+} K^{-}\right) \pi^{+}$decay. The available theoretical predictions are generally consistent with the measurement.
\end{abstract}

\section{Introduction}

The $B_{c}^{+}$meson $^{1}$ is the only known weakly decaying particle consisting of two heavy quarks. The ground $\bar{b} c$ state was first observed by $\mathrm{CDF}$ [1] via its semileptonic decay $B_{c}^{+} \rightarrow$ $J / \psi \ell^{+} v_{\ell}$. An excited $\bar{b} c$ state has been observed recently by ATLAS [2] using the $B_{c}^{+}$decay mode $B_{c}^{+} \rightarrow J / \psi \pi^{+}$. The presence of two heavy quarks, each of which can decay weakly, affects theoretical calculations of the decay proper-

\footnotetext{
${ }^{1}$ Charge conjugate states are implied throughout the paper unless otherwise stated.
}

^e-mail: atlas.publications@cern.ch ties of the $B_{c}^{+}$meson. In the case of $\bar{b} \rightarrow \bar{c} c \bar{s}$ processes, decays to charmonium and a $D_{s}^{+}$or a $D_{s}^{*+}$ meson are predicted to occur via colour-suppressed and colour-favoured spectator diagrams as well as via the weak annihilation diagram (see Fig. 1). The latter, in contrast to decays of other $B$ mesons, is not Cabibbo-suppressed and can contribute significantly to the decay amplitudes. The decay properties are addressed in various theoretical calculations [3-9] and can also be compared to the analogous properties in the lighter $B$ meson systems such as $B_{d}^{0} \rightarrow D^{*-} D_{s}^{(*)+}$ or $B^{+} \rightarrow \bar{D}^{* 0} D_{s}^{(*)+}$. The decays $B_{c}^{+} \rightarrow J / \psi D_{s}^{+}$and $B_{c}^{+} \rightarrow$ $J / \psi D_{s}^{*+}$, which have been observed recently by the $\mathrm{LHCb}$ experiment [10], provide a means to test these theoretical predictions.

This paper presents a measurement of the branching fractions of $B_{c}^{+} \rightarrow J / \psi D_{s}^{+}$and $B_{c}^{+} \rightarrow J / \psi D_{s}^{*+}$ decays, normalised to that of $B_{c}^{+} \rightarrow J / \psi \pi^{+}$decay, and polarisation in $B_{c}^{+} \rightarrow J / \psi D_{s}^{*+}$ decay performed with the ATLAS detector [11]. The $D_{s}^{+}$meson is reconstructed via the $D_{s}^{+} \rightarrow \phi \pi^{+}$ decay with the $\phi$ meson decaying into a pair of charged kaons. The $D_{s}^{*+}$ meson decays into a $D_{s}^{+}$meson and a soft photon or $\pi^{0}$. Detecting such soft neutral particles is very challenging, thus no attempt to reconstruct them is made in the analysis. The $J / \psi$ meson is reconstructed via its decay into a muon pair.

The measurement presented in this paper allows an independent verification of the results of Ref. [10] with comparable statistical and systematic uncertainties. The following ratios are measured: $\mathcal{R}_{D_{s}^{+} / \pi^{+}}=\mathcal{B}_{B_{c}^{+} \rightarrow J / \psi D_{s}^{+}} / \mathcal{B}_{B_{c}^{+} \rightarrow J / \psi \pi^{+}}$, $\mathcal{R}_{D_{s}^{*+} / \pi^{+}}=\mathcal{B}_{B_{c}^{+} \rightarrow J / \psi D_{s}^{*+}} / \mathcal{B}_{B_{c}^{+} \rightarrow J / \psi \pi^{+}}$, and $\mathcal{R}_{D_{s}^{*+} / D_{s}^{+}}=$ $\mathcal{B}_{B_{c}^{+} \rightarrow J / \psi D_{s}^{*+}} / \mathcal{B}_{B_{c}^{+} \rightarrow J / \psi D_{s}^{+}}$, where $\mathcal{B}_{B_{c}^{+} \rightarrow X}$ denotes the branching fraction of the $B_{c}^{+} \rightarrow X$ decay. The decay $B_{c}^{+} \rightarrow J / \psi D_{s}^{*+}$ is a transition of a pseudoscalar meson into a pair of vector states and is thus described by the three helicity amplitudes, $A_{++}, A_{--}$, and $A_{00}$, where the subscripts correspond to the helicities of $J / \psi$ and $D_{s}^{*+}$ mesons. The contribution of the $A_{++}$and $A_{--}$amplitudes, referred to as the $A_{ \pm \pm}$component, corresponds to the $J / \psi$ and $D_{s}^{*+}$ transverse polarisation. The fraction of transverse polarisation, 


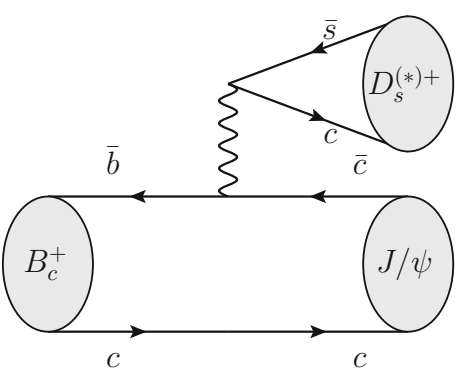

(a)

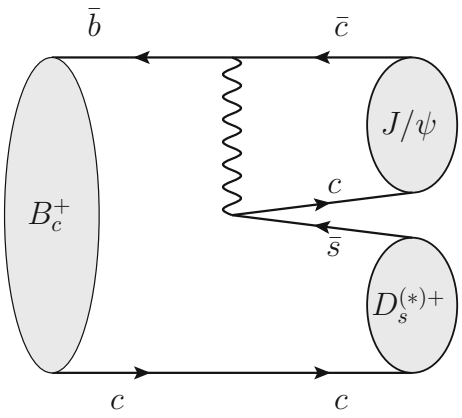

(b)

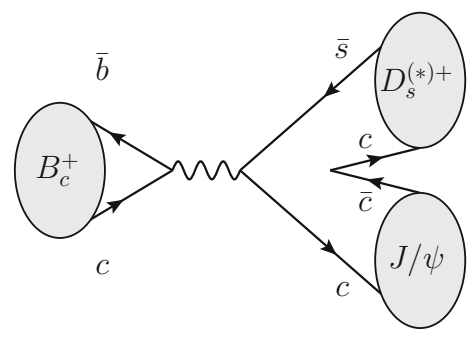

(c)

Fig. 1 Feynman diagrams for $B_{c}^{+} \rightarrow J / \psi D_{s}^{(*)+}$ decays: a colour-favoured spectator, b colour-suppressed spectator, and $\mathbf{c}$ annihilation topology

$\Gamma_{ \pm \pm} / \Gamma=\Gamma_{ \pm \pm}\left(B_{c}^{+} \rightarrow J / \psi D_{s}^{*+}\right) / \Gamma\left(B_{c}^{+} \rightarrow J / \psi D_{s}^{*+}\right)$, is also measured. From a naive prediction by spin counting, one would expect this fraction to be $2 / 3$, while calculations $[8,9]$ predict values of $0.41-0.48$.

This analysis is based on a combined sample of $p p$ collision data collected by the ATLAS experiment at the LHC at centre-of-mass energies $\sqrt{s}=7 \mathrm{TeV}$ and $8 \mathrm{TeV}$ corresponding to integrated luminosities of 4.9 and $20.6 \mathrm{fb}^{-1}$, respectively.

\section{The ATLAS detector, trigger selection and Monte Carlo samples}

ATLAS is a general-purpose detector consisting of several subsystems including the inner detector (ID), calorimeters and the muon spectrometer (MS). Muon reconstruction makes use of both the ID and the MS. The ID comprises three types of detectors: a silicon pixel detector, a silicon microstrip semiconductor tracker (SCT) and a transition radiation tracker. The ID provides a pseudorapidity ${ }^{2}$ coverage up to $|\eta|=2.5$. Muons pass through the calorimeters and reach the MS if their transverse momentum, $p_{\mathrm{T}}$, is above approximately $3 \mathrm{GeV} .{ }^{3}$ Muon candidates are formed either from a stand-alone MS track matched to an ID track or, in case the MS stand-alone track is not reconstructed, from an ID track extrapolated to the MS and matched to track segments in the MS. Candidates of the latter type are referred to as segmenttagged muons while the former are called combined muons. Muon track parameters are taken from the ID measurement alone in this analysis, since the precision of the measured

\footnotetext{
2 ATLAS uses a right-handed coordinate system with its origin at the nominal interaction point (IP) in the centre of the detector and the $z$-axis along the beam pipe. The $x$-axis points from the IP to the centre of the LHC ring, and the $y$-axis points upward. Cylindrical coordinates $(r, \phi)$ are used in the transverse plane, $\phi$ being the azimuthal angle around the beam pipe. The pseudorapidity is defined in terms of the polar angle $\theta$ as $\eta=-\ln \tan (\theta / 2)$.

${ }^{3}$ Using a system of units with $c=1$ is implied throughout the paper.
}

track parameters for muons in the $p_{\mathrm{T}}$ range of interest is dominated by the ID track reconstruction.

The ATLAS trigger system consists of a hardware-based Level-1 trigger and a two-stage high level trigger (HLT). At Level-1, the muon trigger uses dedicated MS chambers to search for patterns of hits satisfying different $p_{\mathrm{T}}$ thresholds. The region-of-interest around these hit patterns then serves as a seed for the HLT muon reconstruction, in which dedicated algorithms are used to incorporate information from both the MS and the ID, achieving a position and momentum resolution close to that provided by the offline muon reconstruction. Muons are efficiently triggered in the pseudorapidity range $|\eta|<2.4$.

Triggers based on single-muon, dimuon, and three-muon signatures are used to select $J / \psi \rightarrow \mu^{+} \mu^{-}$decays for the analysis. The third muon can be produced in the $B_{c}^{+}$signal events in semileptonic decays of the two other heavy-flavour hadrons. The majority of events are collected by dimuon triggers requiring a vertex of two oppositely charged muons with an invariant mass between 2.5 and $4.3 \mathrm{GeV}$. During the data taking, the $p_{\mathrm{T}}$ threshold for muons in these triggers was either 4 or $6 \mathrm{GeV}$. Single-muon triggers additionally increase the acceptance for asymmetric $J / \psi$ decays where one muon has $p_{\mathrm{T}}<4 \mathrm{GeV}$. Finally, three-muon triggers had a $p_{\mathrm{T}}$ threshold of $4 \mathrm{GeV}$, thus enhancing the acceptance during the periods of high luminosity when the $p_{\mathrm{T}}$ threshold for at least one muon in the dimuon triggers was $6 \mathrm{GeV}$.

Monte Carlo (MC) simulation is used for the event selection criteria optimisation and the calculation of the acceptance for the considered $B_{c}^{+}$decay modes. The MC samples of the $B_{c}^{+}$decays were generated with PYTHIA 6.4 [12] along with a dedicated extension for the $B_{c}^{+}$production based on calculations from Refs. [13-16]. The decays of $B_{c}^{+}$are then simulated with EvTGEN [17]. The generated events were passed through a full simulation of the detector using the ATLAS simulation framework [18] based on GEANT $4[19,20]$ and processed with the same reconstruction algorithms as were used for the data. 


\section{Reconstruction and event selection}

The $J / \psi$ candidates are reconstructed from pairs of oppositely charged muons. At least one of the two muons is required to be a combined muon. Each pair is fitted to a common vertex [21]. The quality of the vertex fit must satisfy $\chi^{2} /$ ndf $<15$, where the ndf stands for the number of degrees of freedom. The candidates in the invariant mass window $2800 \mathrm{MeV}<m\left(\mu^{+} \mu^{-}\right)<3400 \mathrm{MeV}$ are retained.

For the $D_{s}^{+} \rightarrow \phi\left(K^{+} K^{-}\right) \pi^{+}$reconstruction, tracks of particles with opposite charges are assigned kaon mass hypotheses and combined in pairs to form $\phi$ candidates. An additional track is assigned a pion mass and combined with the $\phi$ candidate to form a $D_{s}^{+}$candidate. To ensure good momentum resolution, all three tracks are required to have at least two hits in the silicon pixel detector and at least six hits in the SCT. Only three-track combinations successfully fitted to a common vertex with $\chi^{2} /$ ndf $<8$ are kept. The $\phi$ candidate invariant mass, $m\left(K^{+} K^{-}\right)$, and the $D_{s}^{+}$candidate invariant mass, $m\left(K^{+} K^{-} \pi^{+}\right)$, are calculated using the track momenta refitted to the common vertex. Only candidates with $m\left(K^{+} K^{-}\right)$within $\pm 7 \mathrm{MeV}$ around the $\phi$ mass, $m_{\phi}=1019.461 \mathrm{MeV}$ [22], and with $1930 \mathrm{MeV}<m\left(K^{+} K^{-} \pi^{+}\right)<2010 \mathrm{MeV}$ are retained.

The $B_{c}^{+} \rightarrow J / \psi D_{s}^{+}$candidates are built by combining the five tracks of the $J / \psi$ and $D_{s}^{+}$candidates. The $J / \psi$ meson decays instantly at the same point as the $B_{c}^{+}$does (secondary vertex) while the $D_{s}^{+}$lives long enough to form a displaced tertiary vertex. Therefore the five-track combinations are refitted assuming this cascade topology [21]. The invariant mass of the muon pair is constrained to the $J / \psi$ mass, $m_{J / \psi}=3096.916 \mathrm{MeV}$ [22]. The three $D_{s}^{+}$daughter tracks are constrained to a tertiary vertex and their invariant mass is fixed to the mass of $D_{s}^{+}, m_{D_{s}^{+}}=1968.30 \mathrm{MeV}$ [22]. The combined momentum of the refitted $D_{s}^{+}$decay tracks is constrained to point to the dimuon vertex. The quality of the cascade fit must satisfy $\chi^{2} /$ ndf $<3$.

The $B_{c}^{+}$meson is reconstructed within the kinematic range $p_{\mathrm{T}}\left(B_{c}^{+}\right)>15 \mathrm{GeV}$ and $\left|\eta\left(B_{c}^{+}\right)\right|<2.0$, where the detector acceptance is high and depends weakly on $p_{\mathrm{T}}\left(B_{c}^{+}\right)$and $\eta\left(B_{c}^{+}\right)$.

The refitted tracks of the $D_{s}^{+}$daughter hadrons are required to have $|\eta|<2.5$ and $p_{\mathrm{T}}>1 \mathrm{GeV}$, while the muons must have $|\eta|<2.3$ and $p_{\mathrm{T}}>3 \mathrm{GeV}$. To further discriminate the sample of $D_{s}^{+}$candidates from a large combinatorial background, the following requirements are applied:

- $\cos \theta^{*}(\pi)<0.8$, where $\theta^{*}(\pi)$ is the angle between the pion momentum in the $K^{+} K^{-} \pi^{+}$rest frame and the $K^{+} K^{-} \pi^{+}$combined momentum in the laboratory frame;

- $\left|\cos ^{3} \theta^{\prime}(K)\right|>0.15$, where $\theta^{\prime}(K)$ is the angle between one of the kaons and the pion in the $K^{+} K^{-}$rest frame.
The decay of the pseudoscalar $D_{s}^{+}$meson to the $\phi$ (vector) plus $\pi$ (pseudoscalar) final state results in an alignment of the spin of the $\phi$ meson perpendicularly to the direction of motion of the $\phi$ relative to $D_{s}^{+}$. Consequently, the distribution of $\cos \theta^{\prime}(K)$ follows a $\cos ^{2} \theta^{\prime}(K)$ shape, implying a uniform distribution for $\cos ^{3} \theta^{\prime}(K)$. In contrast, the $\cos \theta^{\prime}(K)$ distribution of the combinatorial background is uniform and its $\cos ^{3} \theta^{\prime}(K)$ distribution peaks at zero. The cut suppresses the background significantly while reducing the signal by $15 \%$.

The $B_{c}^{+}$candidate is required to point back to a primary vertex such that $d_{0}^{\mathrm{PV}}\left(B_{c}^{+}\right)<0.1 \mathrm{~mm}$ and $z_{0}^{\mathrm{PV}}\left(B_{c}^{+}\right) \sin \theta\left(B_{c}^{+}\right)$ $<0.5 \mathrm{~mm}$, where $d_{0}^{\mathrm{PV}}$ and $z_{0}^{\mathrm{PV}}$ are respectively the transverse and longitudinal impact parameters with respect to the primary vertex. All primary vertices in the event are considered. If there is more than one primary vertex satisfying these requirements $(\sim 0.5 \%$ events both in data and MC simulation), the one with the largest sum of squared transverse momenta of the tracks originating from it is chosen.

The transverse decay length ${ }^{4}$ of the $B_{c}^{+}$candidate is required to satisfy $L_{x y}\left(B_{c}^{+}\right)>0.1 \mathrm{~mm}$. The transverse decay length of the $D_{s}^{+}$measured from the $B_{c}^{+}$vertex must be $L_{x y}\left(D_{s}^{+}\right)>0.15 \mathrm{~mm}$. In order to remove fake candidates, both $L_{x y}\left(B_{c}^{+}\right)$and $L_{x y}\left(D_{s}^{+}\right)$are required not to exceed $10 \mathrm{~mm}$.

Taking into account the characteristic hard fragmentation of $b$-quarks, a requirement $p_{\mathrm{T}}\left(B_{c}^{+}\right) / \sum p_{\mathrm{T}}(\operatorname{trk})>0.1$ is applied, where the sum in the denominator is taken over all tracks originating from the primary vertex (tracks of the $B_{c}^{+}$ candidate are included in the sum if they are associated with the primary vertex). The requirement reduces a sizeable fraction of combinatorial background while having almost no effect on the signal.

The following angular selection requirements are introduced to further suppress the combinatorial background:

- $\cos \theta^{*}\left(D_{s}^{+}\right)>-0.8$, where $\theta^{*}\left(D_{s}^{+}\right)$is the angle between the $D_{s}^{+}$candidate momentum in the rest frame of the $B_{c}^{+}$candidate, and the $B_{c}^{+}$candidate line of flight in the laboratory frame. The distribution of $\cos \theta^{*}\left(D_{s}^{+}\right)$is uniform for the decays of pseudoscalar $B_{c}^{+}$meson before any kinematic selection while it tends to increase for negative values of $\cos \theta^{*}\left(D_{s}^{+}\right)$for the background.

- $\cos \theta^{\prime}(\pi)>-0.8$, where $\theta^{\prime}(\pi)$ is the angle between the $J / \psi$ candidate momentum and the pion momentum in the $K^{+} K^{-} \pi^{+}$rest frame. Its distribution is nearly uniform for the signal processes but peaks towards -1 for the background.

\footnotetext{
4 The transverse decay length of a particle is defined as the transverse distance between the production (primary) vertex and the particle decay (secondary) vertex projected along its transverse momentum.
} 


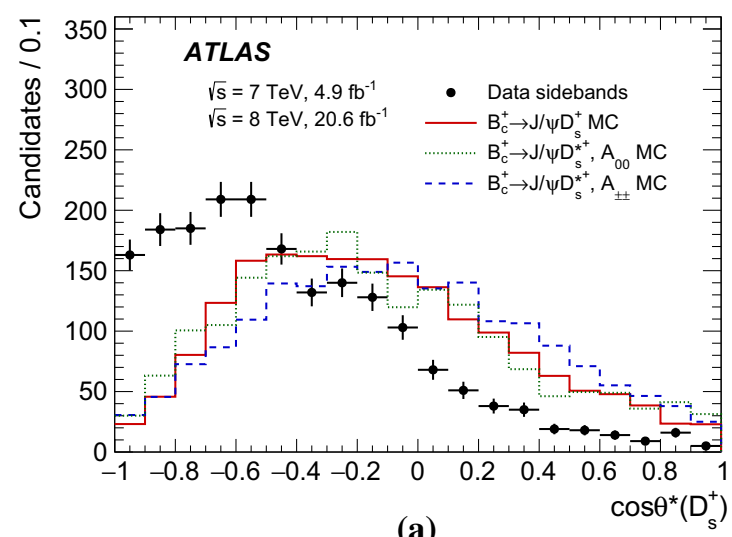

(a)

Fig. 2 Distributions of $\mathbf{a} \cos \theta^{*}\left(D_{s}^{+}\right)$and $\mathbf{b} \cos \theta^{\prime}(\pi)$, where $\theta^{*}\left(D_{s}^{+}\right)$ and $\theta^{\prime}(\pi)$ are two angular variables defined in Sect. 3. The distributions are shown for data sidebands (black dots) and MC simulation of $B_{c}^{+} \rightarrow J / \psi D_{s}^{+}$signal (red solid line) and $A_{00}$ (green dotted line) and

Distributions of these two variables after applying all other selection requirements described in this section are shown in Fig. 2. They are shown for the simulated signal samples, as well as for sidebands of the mass spectrum in data, defined as the regions $5640 \mathrm{MeV}<m\left(J / \psi D_{s}^{+}\right)<5900 \mathrm{MeV}$ (left sideband) and $6360 \mathrm{MeV}<m\left(J / \psi D_{s}^{+}\right)<6760 \mathrm{MeV}$ (right sideband). A dip in the $\cos \theta^{\prime}(\pi)$ distribution for the $B_{c}^{+} \rightarrow J / \psi D_{s}^{+}$signal is caused by rejection of $B_{s}^{0} \rightarrow J / \psi \phi$ candidates discussed below.

Various possible contributions of partially reconstructed $B \rightarrow J / \psi X$ decays were studied. The only significant one was found from the $B_{s}^{0} \rightarrow J / \psi \phi$ decay process. This contribution arises when the combination of the tracks from a true $B_{s}^{0} \rightarrow J / \psi\left(\mu^{+} \mu^{-}\right) \phi\left(K^{+} K^{-}\right)$decay with a fifth random track results in a fake $B_{c}^{+} \rightarrow J / \psi\left(\mu^{+} \mu^{-}\right) D_{s}^{+}\left(K^{+} K^{-} \pi^{+}\right)$ candidate. For each reconstructed $B_{c}^{+}$candidate, an additional vertex fit is performed. The two muon tracks and the two kaon tracks are fitted to a common vertex, where the kaon tracks are assumed to be from $\phi \rightarrow K^{+} K^{-}$and the muon pair is constrained to have the nominal $J / \psi$ mass. The mass of the $B_{s}^{0}$ candidate, $m\left(\mu^{+} \mu^{-} K^{+} K^{-}\right)$, is then calculated from the refitted track parameters. Candidates with $5340 \mathrm{MeV}<m\left(\mu^{+} \mu^{-} K^{+} K^{-}\right)<5400 \mathrm{MeV}$ are rejected. This requirement suppresses the bulk of the $B_{s}^{0}$ events while rejecting only $\sim 4 \%$ of the signal.

After applying the selection requirements described above, $1547 \mathrm{~J} / \psi D_{s}^{+}$candidates are selected in the mass range 5640-6760 MeV.

\section{$4 B_{c}^{+} \rightarrow J / \psi D_{s}^{(*)+}$ candidate fit}

The mass distribution of the selected $B_{c}^{+} \rightarrow J / \psi D_{s}^{(*)+}$ candidates is shown in Fig. 3. The peak near the $B_{c}^{+}$mass, $m_{B_{c}^{+}}=6275.6 \mathrm{MeV}$ [22], is attributed to the signal of

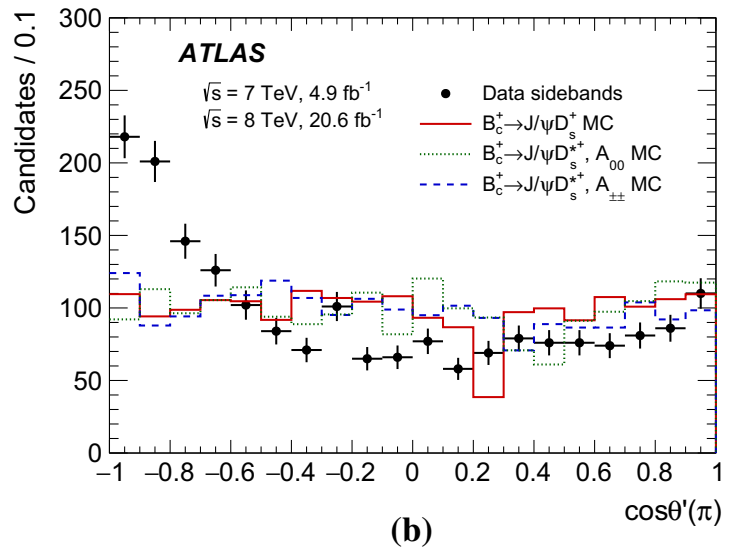

$A_{ \pm \pm}$(blue dashed line) components of $B_{c}^{+} \rightarrow J / \psi D_{s}^{*+}$ signal. The distributions are obtained after applying all selection criteria except the ones on the plotted variable. The MC distributions are normalised to data

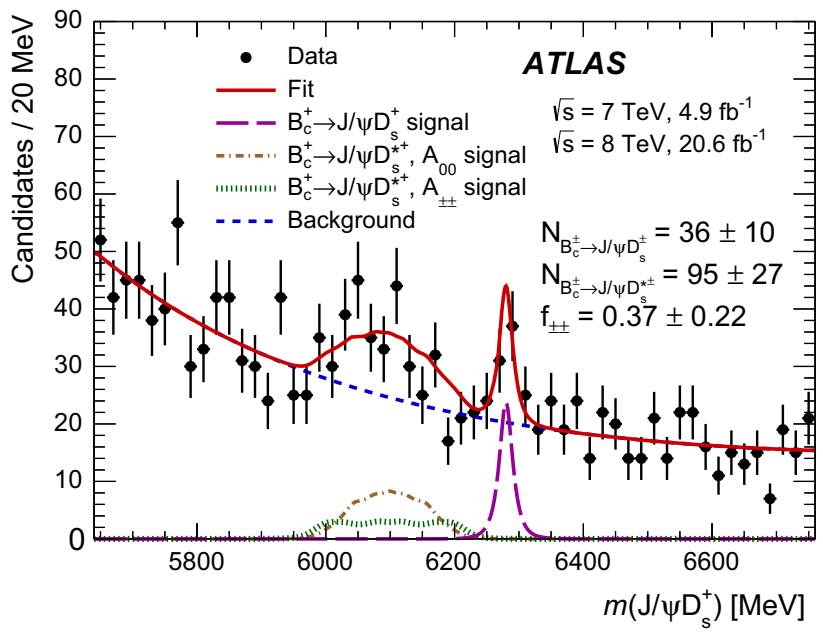

Fig. 3 The mass distribution for the selected $J / \psi D_{s}^{+}$candidates. The red solid line represents the projection of the fit to the model described in the text. The contribution of the $B_{c}^{+} \rightarrow J / \psi D_{s}^{+}$decay is shown with the magenta long-dashed line; the brown dash-dot and green dotted lines show the $B_{c}^{+} \rightarrow J / \psi D_{s}^{*+} A_{00}$ and $A_{ \pm \pm}$component contributions, respectively; the blue dashed line shows the background model. The uncertainties of the listed fit result values are statistical only

$B_{c}^{+} \rightarrow J / \psi D_{s}^{+}$decay while a wider structure between 5900 and $6200 \mathrm{MeV}$ corresponds to $B_{c}^{+} \rightarrow J / \psi D_{s}^{*+}$ with subsequent $D_{s}^{*+} \rightarrow D_{s}^{+} \gamma$ or $D_{s}^{*+} \rightarrow D_{s}^{+} \pi^{0}$ decays where the neutral particle is not reconstructed.

Mass distributions of the $J / \psi$ and $D_{s}^{+}$candidates corresponding to the $J / \psi D_{s}^{+}$mass region of the observed $B_{c}^{+} \rightarrow J / \psi D_{s}^{(*)+}$ signals are shown in Fig. 4. To obtain these plots, the $B_{c}^{+}$candidates are built without the mass constraints in the cascade fit, with the mass of the candidate calculated as $m\left(J / \psi D_{s}^{+}\right)=m\left(\mu^{+} \mu^{-} K^{+} K^{-} \pi^{+}\right)-$ $m\left(\mu^{+} \mu^{-}\right)+m_{J / \psi}-m\left(K^{+} K^{-} \pi^{+}\right)+m_{D_{s}^{+}}$, where $m_{J / \psi}$ and $m_{D_{s}^{+}}$are the nominal masses of the respective par- 


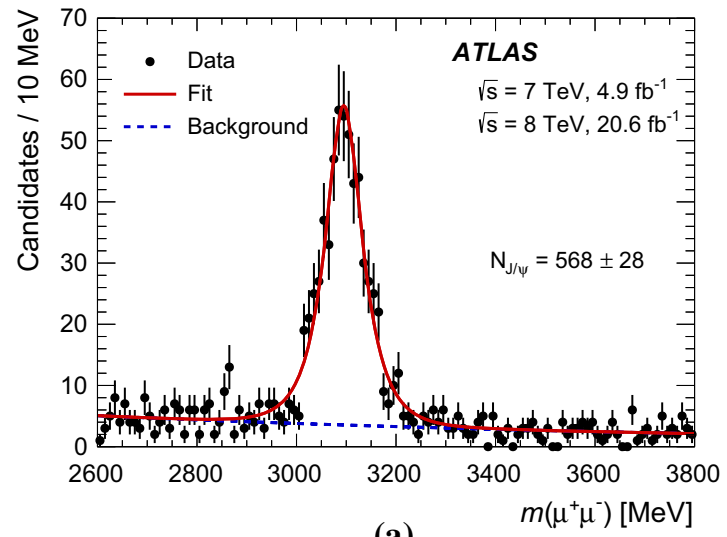

(a)

Fig. 4 Mass distribution of the a $J / \psi$ and $\mathbf{b} D_{s}^{+}$candidates after the full $B_{c}^{+} \rightarrow J / \psi D_{s}^{(*)+}$ selection (without mass constraints in the cascade fit) in the mass window of the $B_{c}^{+}$candidate $5900 \mathrm{MeV}<$

ticles. The mass of the $B_{c}^{+}$candidate is required to be $5900 \mathrm{MeV}<m\left(J / \psi D_{s}^{+}\right)<6400 \mathrm{MeV}$ while the mass windows for the corresponding intermediate resonances are widened to the plotting ranges. The $J / \psi$ and $D_{s}^{+}$mass distributions are fitted with a sum of an exponential function describing the background and a modified Gaussian function $[23,24]$ describing the corresponding signal peak. The modified Gaussian function is defined as

Gauss $^{\bmod } \sim \exp \left(-\frac{x^{1+\frac{1}{1+x / 2}}}{2}\right)$,

where $x=\left|m_{0}-m\right| / \sigma$ with the mean mass $m_{0}$ and width $\sigma$ being free parameters. The fitted masses of $J / \psi$ $(3095.1 \pm 2.4 \mathrm{MeV})$ and $D_{s}(1969.0 \pm 4.1 \mathrm{MeV})$ agree with their nominal masses, the widths are consistent with those in the simulated samples, and the signal yields are found to be $N_{J / \psi}=568 \pm 28$ and $N_{D_{s}^{ \pm}}=175 \pm 36$.

The information about the helicity in $B_{c}^{+} \rightarrow J / \psi D_{s}^{*+}$ decay is encoded both in the mass distribution of the $J / \psi D_{s}^{+}$ system and in the distribution of the helicity angle, $\theta^{\prime}\left(\mu^{+}\right)$, which is defined in the rest frame of the muon pair as the angle between the $\mu^{+}$and the $D_{s}^{+}$candidate momenta. Thus, a two-dimensional extended unbinned maximum-likelihood fit of the $m\left(J / \psi D_{s}^{+}\right)$and $\left|\cos \theta^{\prime}\left(\mu^{+}\right)\right|$distributions is performed. The $A_{++}$and $A_{--}$helicity amplitude contributions are described by the same mass and angular shapes because of the parity symmetry of the $J / \psi$ and $D_{s}^{*+}$ decays. This is confirmed by the MC simulation. Thus these components are treated together as the $A_{ \pm \pm}$component, while the shape of the $A_{00}$ component is different and is therefore treated separately. A simultaneous fit to the mass and angular distributions significantly improves the sensitivity to the contributions of the helicity amplitudes in $B_{c}^{+} \rightarrow J / \psi D_{s}^{*+}$ decay with respect to a one-dimensional mass fit.

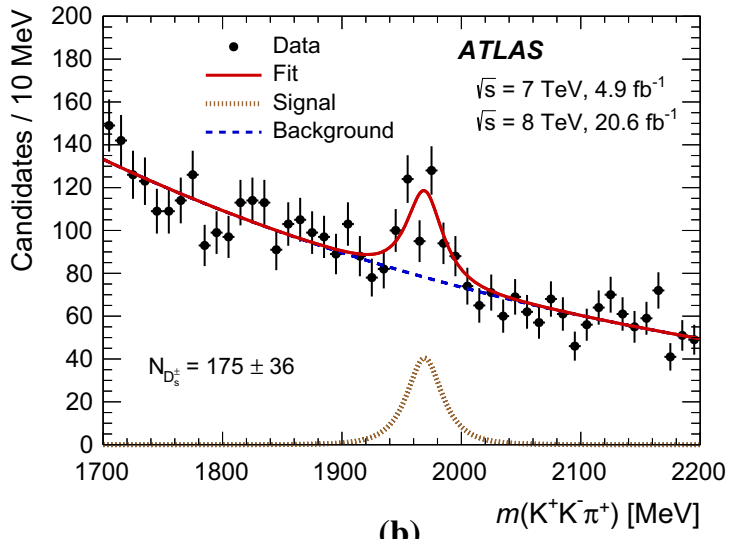

(b)

$m\left(J / \psi D_{s}^{+}\right)<6400 \mathrm{MeV}$. The spectra are fitted with a sum of an exponential and a modified Gaussian function. The uncertainties of the shown $J / \psi$ and $D_{s}^{+}$yields are statistical only

Four two-dimensional probability density functions (PDFs) are defined to describe the $B_{c}^{+} \rightarrow J / \psi D_{s}^{+}$signal, the $A_{ \pm \pm}$and $A_{00}$ components of the $B_{c}^{+} \rightarrow J / \psi D_{s}^{*+}$ signal, and the background. The signal PDFs are factorised into mass and angular components. The effect of correlations between their mass and angular shapes is found to be small and is accounted for as a systematic uncertainty.

The mass distribution of the $B_{c}^{+} \rightarrow J / \psi D_{s}^{+}$signal is described by a modified Gaussian function. For the $B_{c}^{+} \rightarrow$ $J / \psi D_{s}^{*+}$ signal components, the mass shape templates obtained from the simulation with the kernel estimation technique [25] are used. The branching fractions of $D_{s}^{*+} \rightarrow$ $D_{s}^{+} \pi^{0}$ and $D_{s}^{*+} \rightarrow D_{s}^{+} \gamma$ decays for the simulation are set to the world average values [22]. The position of the templates along the mass axis is varied in the fit simultaneously with the position of the $B_{c}^{+} \rightarrow J / \psi D_{s}^{+}$signal peak. The background mass shape is described with a two-parameter exponential function, $\exp \left[a \cdot m\left(J / \psi D_{s}^{+}\right)+b \cdot m\left(J / \psi D_{s}^{+}\right)^{2}\right]$.

To describe the $\left|\cos \theta^{\prime}\left(\mu^{+}\right)\right|$shapes, templates from the kernel estimation are used. The templates for the signal angular PDFs are extracted from the simulated samples. Although their shapes are calculable analytically, using the templates allows the fit to account for detector effects. The background angular description is based on the $\left|\cos \theta^{\prime}\left(\mu^{+}\right)\right|$ shape of the candidates in the sidebands of $J / \psi D_{s}^{+}$mass spectra. Two templates are produced from the angular distributions of the candidates in the left and right mass sidebands as defined in Sect. 3. The angular PDF for the background is defined as a conditional PDF of $\left|\cos \theta^{\prime}\left(\mu^{+}\right)\right|$ given the per-candidate $m\left(J / \psi D_{s}^{+}\right)$. For the candidates in the lower half of the left sideband $(5640-5770 \mathrm{MeV})$, the template from the left sideband is used. Similarly, the template from the right sideband is used for the upper half of the right sideband $(6560-6760 \mathrm{MeV})$. For the candidates in the middle part of the mass spectrum (5770- 
Table 1 Parameters of the $B_{c}^{+} \rightarrow J / \psi D_{s}^{(*)+}$ signals obtained with the unbinned extended maximum-likelihood fit. The width parameter of the modified Gaussian function is fixed to the MC value. Only statistical uncertainties are shown. No acceptance corrections are applied to the signal yields

\begin{tabular}{lc}
\hline Parameter & Value \\
\hline$m_{B_{c}^{+} \rightarrow J / \psi D_{s}^{+}}(\mathrm{MeV})$ & $6279.9 \pm 3.5$ \\
$N_{B_{c}^{+} \rightarrow J / \psi D_{s}^{+}}$ & $36 \pm 10$ \\
$N_{B_{c}^{+} \rightarrow J / \psi D_{s}^{*+}}$ & $95 \pm 27$ \\
$f_{ \pm \pm}$ & $0.37 \pm 0.22$ \\
\hline
\end{tabular}

$6560 \mathrm{MeV}$ ), a linear interpolation between the two templates is used.

The fit has seven free parameters: the mass of the $B_{c}^{+}$ meson, $m_{B_{c}^{+} \rightarrow J / \psi D_{s}^{+}}$; the relative contribution of the $A_{ \pm \pm}$ component to the total $B_{c}^{+} \rightarrow J / \psi D_{s}^{*+}$ decay rate in the selected sample, $f_{ \pm \pm}$; the two parameters of the exponential background; the yields of the two signal modes, $N_{B_{c}^{+} \rightarrow J / \psi D_{s}^{+}}$and $N_{B_{c}^{+} \rightarrow J / \psi D_{s}^{*+}}$, and the background yield. The width of the modified Gaussian function, $\sigma_{B_{c}^{+} \rightarrow J / \psi D_{s}^{+}}$, is fixed to the value obtained from the fit to the simulated signal, $\sigma_{B_{c}^{+} \rightarrow J / \psi D_{s}^{+}}=9.95 \mathrm{MeV}$. Leaving this parameter free in the data fit results in the value $7.9 \pm 3.0 \mathrm{MeV}$, consistent with the simulation in the range of statistical uncertainty.

It was checked that the fit procedure provides unbiased values and correct statistical uncertainties for the extracted parameters using pseudo-experiments. The values of the relevant parameters obtained from the fit are given in Table 1. The fitted $B_{c}^{+}$mass agrees with the world average value [22].

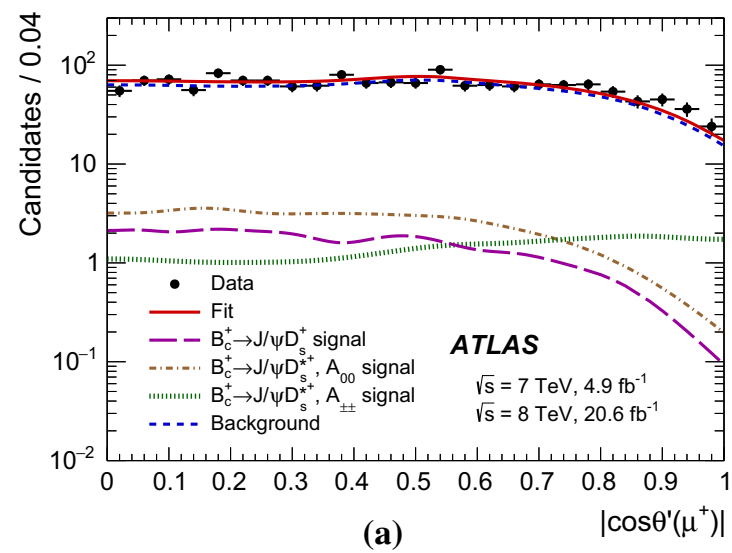

Fig. 5 The projection of the likelihood fit on the variable $\left|\cos \theta^{\prime}\left(\mu^{+}\right)\right|$, where the helicity angle $\theta^{\prime}\left(\mu^{+}\right)$is the angle between the $\mu^{+}$and $D_{s}^{+}$ candidate momenta in the rest frame of the muon pair from $J / \psi$ decay, for $\mathbf{a}$ the full selected $J / \psi D_{s}^{+}$candidate dataset and $\mathbf{b}$ a subset of the candidates in a mass range $5950 \mathrm{MeV}<m\left(J / \psi D_{s}^{+}\right)<6250 \mathrm{MeV}$ corresponding to the observed signal of $B_{c}^{+} \rightarrow J / \psi D_{s}^{*+}$ decay. The
The mass and angular projections of the fit on the selected $J / \psi D_{s}^{+}$candidate dataset are also shown in Figs. 3 and 5a, respectively. In order to illustrate the effect of the angular part of the fit in separating the helicity amplitudes, the $\left|\cos \theta^{\prime}\left(\mu^{+}\right)\right|$projection for the subset of candidates with the masses $5950 \mathrm{MeV}<m\left(J / \psi D_{s}^{+}\right)<6250 \mathrm{MeV}$ corresponding to the region of the observed $B_{c}^{+} \rightarrow J / \psi D_{s}^{*+}$ signal is shown in Fig. 5b.

The statistical significance for the observed $B_{c}^{+}$signal estimated from toy MC studies is 4.9 standard deviations.

\section{$5 B_{c}^{+} \rightarrow J / \psi \pi^{+}$candidate reconstruction and fit}

$B_{c}^{+} \rightarrow J / \psi \pi^{+}$candidates are reconstructed by fitting a common vertex of a pion candidate track and the two muons from a $J / \psi$ candidate, selected as described in Sect. 3. For the pion candidate, tracks identified as muons are vetoed in order to suppress the substantial background from $B_{c}^{+} \rightarrow$ $J / \psi \mu^{+} v_{\mu} X$ decays. The invariant mass of the two muons in the vertex fit is constrained to the $J / \psi$ nominal mass. The quality of the fit must satisfy $\chi^{2} /$ ndf $<3$. The following selection requirements applied to the $B_{c}^{+} \rightarrow J / \psi \pi^{+}$ candidates are analogous to those for $B_{c}^{+} \rightarrow J / \psi D_{s}^{+}$candidates described in Sect. 3: the candidates must be within the kinematic range $p_{\mathrm{T}}\left(B_{c}^{+}\right)>15 \mathrm{GeV},\left|\eta\left(B_{c}^{+}\right)\right|<2.0$; the refitted values of the transverse momenta and pseudorapidities of the muons are required to satisfy $p_{\mathrm{T}}\left(\mu^{ \pm}\right)>3 \mathrm{GeV}$, $\left|\eta\left(\mu^{ \pm}\right)\right|<2.3$; the same requirements on pointing to the primary vertex and the ratio $p_{\mathrm{T}}\left(B_{c}^{+}\right) / \sum p_{\mathrm{T}}($ trk $)$ are applied. The refitted pion track kinematics must satisfy $p_{\mathrm{T}}\left(\pi^{+}\right)>$ $5 \mathrm{GeV}$ and $\left|\eta\left(\pi^{+}\right)\right|<2.5$. The transverse decay length

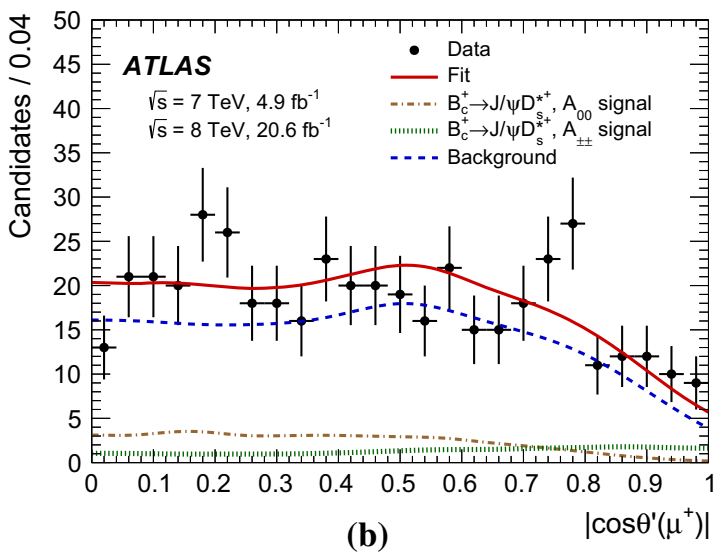

red solid line represents the full fit projection. The contribution of the $B_{c}^{+} \rightarrow J / \psi D_{s}^{+}$decay is shown with the magenta long-dashed line (it is not drawn in b because this contribution vanishes in that mass range); the brown dash-dot and green dotted lines show the $B_{c}^{+} \rightarrow J / \psi D_{s}^{*+}$ $A_{00}$ and $A_{ \pm \pm}$component contributions, respectively; the blue dashed line shows the background model 


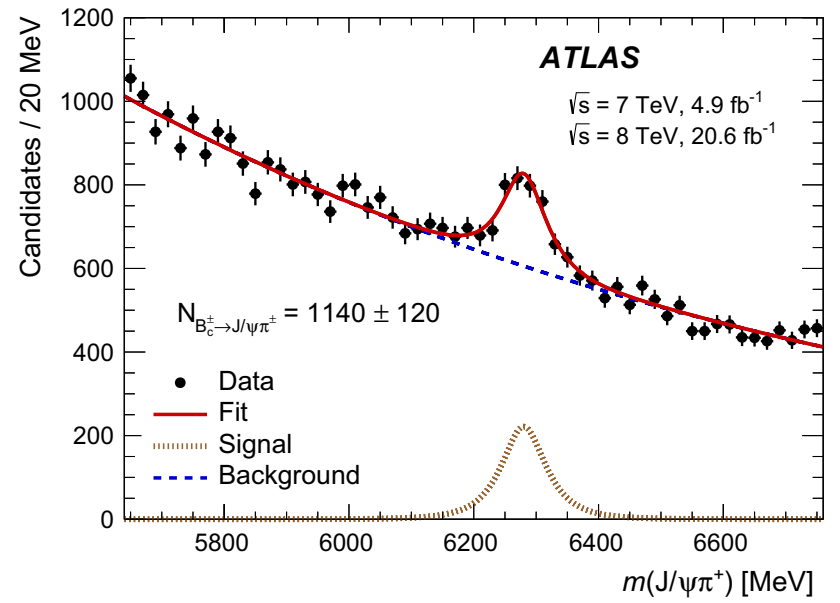

Fig. 6 The mass distribution for the selected $B_{c}^{+} \rightarrow J / \psi \pi^{+}$candidates. The red solid line represents the result of the fit to the model described in the text. The brown dotted and blue dashed lines show the signal and background component projections, respectively. The uncertainty of the shown signal yield is statistical only

is required to be $L_{x y}\left(B_{c}^{+}\right)>0.2 \mathrm{~mm}$, and not to exceed $10 \mathrm{~mm}$.

To further suppress combinatorial background, the following selection is applied:

- $\cos \theta^{*}(\pi)>-0.8$, where $\theta^{*}(\pi)$ is the angle between the pion momentum in the $\mu^{+} \mu^{-} \pi^{+}$rest frame and the $B_{c}^{+}$ candidate line of flight in laboratory frame. This angular variable behaviour for the signal and the background is the same as that of $\cos \theta^{*}\left(D_{s}^{+}\right)$used for $J / \psi D_{s}^{+}$candidates selection.

- $\left|\cos \theta^{\prime}\left(\mu^{+}\right)\right|<0.8$, where $\theta^{\prime}\left(\mu^{+}\right)$is the angle between the $\mu^{+}$and $\pi^{+}$momenta in the muon pair rest frame. The signal distribution follows a $\sin ^{2} \theta^{\prime}\left(\mu^{+}\right)$shape, while the background is flat.

After applying the above-mentioned requirements, 38542 $J / \psi \pi^{+}$candidates are selected in the mass range 5640$6760 \mathrm{MeV}$. Figure 6 shows the mass distribution of the selected candidates. An extended unbinned maximumlikelihood fit of the mass spectrum is performed to evaluate the $B_{c}^{+} \rightarrow J / \psi \pi^{+}$signal yield. The signal contribution is described with the modified Gaussian function while an exponential function is used for the background. The $B_{c}^{+}$ mass, $m_{B_{c}^{+} \rightarrow J / \psi \pi^{+}}$, the width of the modified Gaussian function, $\sigma_{B_{c}^{+} \rightarrow J / \psi \pi^{+}}$, the yields of the signal, $N_{B_{c}^{+} \rightarrow J / \psi \pi^{+}}$, and the background, and the slope of the exponential background are free parameters of the fit. The fit results are summarised in Table 2, and the fit projection is also shown in Fig. 6. The extracted $B_{c}^{+}$mass value is consistent with the world average [22], and the signal peak width agrees with the simulation (37.4 MeV).
Table 2 Signal parameters of the $J / \psi \pi^{+}$mass distribution obtained with the unbinned extended maximum-likelihood fit. Only statistical uncertainties are shown. No acceptance corrections are applied to the signal yields

\begin{tabular}{lr}
\hline Parameter & \multicolumn{1}{l}{ Value } \\
\hline$m_{B_{c}^{+} \rightarrow J / \psi \pi^{+}}(\mathrm{MeV})$ & $6279.9 \pm 3.9$ \\
$\sigma_{B_{c}^{+} \rightarrow J / \psi \pi^{+}}(\mathrm{MeV})$ & $33.9 \pm 4.2$ \\
$N_{B_{c}^{+} \rightarrow J / \psi \pi^{+}}$ & $1140 \pm 120$ \\
\hline
\end{tabular}

\section{Branching fractions and polarisation measurement}

The ratios of the branching fractions $\mathcal{R}_{D_{s}^{+} / \pi^{+}}$and $\mathcal{R}_{D_{s}^{*+} / \pi^{+}}$ are calculated as

$$
\begin{aligned}
\mathcal{R}_{D_{s}^{(*)+} / \pi^{+}}= & \frac{\mathcal{B}_{B_{c}^{+} \rightarrow J / \psi D_{s}^{(*)+}}}{\mathcal{B}_{B_{c}^{+} \rightarrow J / \psi \pi^{+}}}=\frac{1}{\mathcal{B}_{D_{s}^{+} \rightarrow \phi\left(K^{+} K^{-}\right) \pi^{+}}} \\
& \times \frac{\mathcal{A}_{B_{c}^{+} \rightarrow J / \psi \pi^{+}}}{\mathcal{A}_{B_{c}^{+} \rightarrow J / \psi D_{s}^{(*)+}}} \times \frac{N_{B_{c}^{+} \rightarrow J / \psi D_{s}^{(*)+}}}{N_{B_{c}^{+} \rightarrow J / \psi \pi^{+}}},
\end{aligned}
$$

where $\mathcal{A}_{B_{C}^{+} \rightarrow X}$ and $N_{B_{C}^{+} \rightarrow X}$ are the total acceptance and the yield of the corresponding mode. For $\mathcal{B}_{D_{s}^{+} \rightarrow \phi\left(K^{+} K^{-}\right) \pi^{+}}$, the CLEO measurement [26] of the partial $D_{s}^{+} \rightarrow K^{+} K^{-} \pi^{+}$ branching fractions, with a kaon-pair mass within various intervals around the nominal $\phi$ meson mass, is used. An interpolation between the partial branching fractions, measured for \pm 5 and $\pm 10 \mathrm{MeV}$ intervals, using a relativistic Breit-Wigner shape of the resonance yields the value $(1.85 \pm 0.11) \%$ for the $\pm 7 \mathrm{MeV}$ interval which is used in the analysis. The effect of admixture of other $D_{s}^{+}$decay modes with $\left(K^{+} K^{-} \pi^{+}\right)$final state which are not present in the MC simulation is studied separately and accounted for as a systematic uncertainty.

The acceptance for the $B_{c}^{+} \rightarrow J / \psi D_{s}^{*+}$ decay mode is different for the $A_{ \pm \pm}$and $A_{00}$ components, thus the full acceptance for the mode is

$$
\begin{aligned}
& \mathcal{A}_{B_{c}^{+} \rightarrow J / \psi D_{s}^{*+}} \\
& =\left(\frac{f_{ \pm \pm}}{\mathcal{A}_{B_{c}^{+} \rightarrow J / \psi D_{s}^{*+}, A_{ \pm \pm}}}+\frac{1-f_{ \pm \pm}}{\mathcal{A}_{B_{c}^{+} \rightarrow J / \psi D_{s}^{*+}, A_{00}}}\right)^{-1}
\end{aligned}
$$

where the subscripts indicate the helicity state and $f_{ \pm \pm}$is the value extracted from the fit (Table 1). The acceptances are determined from the simulation and shown in Table 3.

The ratio $\mathcal{R}_{D_{s}^{*+} / D_{s}^{+}}$is calculated as

$$
\begin{aligned}
\mathcal{R}_{D_{s}^{*+} / D_{s}^{+}} & =\frac{\mathcal{B}_{B_{c}^{+} \rightarrow J / \psi D_{s}^{*+}}}{\mathcal{B}_{B_{c}^{+} \rightarrow J / \psi D_{s}^{+}}} \\
& =\frac{N_{B_{c}^{+} \rightarrow J / \psi D_{s}^{*+}}}{N_{B_{c}^{+} \rightarrow J / \psi D_{s}^{+}}} \times \frac{\mathcal{A}_{B_{c}^{+} \rightarrow J / \psi D_{s}^{+}}}{\mathcal{A}_{B_{c}^{+} \rightarrow J / \psi D_{s}^{*+}}},
\end{aligned}
$$


Table 3 The acceptance $\mathcal{A}_{B_{c}^{+} \rightarrow X}$ for all decay modes studied. Only uncertainties due to MC statistics are shown

\begin{tabular}{ll}
\hline Mode & $\mathcal{A}_{B_{c}^{+} \rightarrow X}(\%)$ \\
\hline$B_{c}^{+} \rightarrow J / \psi \pi^{+}$ & $4.106 \pm 0.056$ \\
$B_{c}^{+} \rightarrow J / \psi D_{s}^{+}$ & $1.849 \pm 0.034$ \\
$B_{c}^{+} \rightarrow J / \psi D_{s}^{*+}, A_{00}$ & $1.829 \pm 0.053$ \\
$B_{c}^{+} \rightarrow J / \psi D_{s}^{*+}, A_{ \pm \pm}$ & $1.712 \pm 0.035$ \\
\hline
\end{tabular}

where the ratio of the yields $N_{B_{c}^{+} \rightarrow J / \psi D_{s}^{*+}} / N_{B_{c}^{+} \rightarrow J / \psi D_{s}^{+}}$and its uncertainty is extracted from the fit as a parameter in order to account for correlations between the yields.

The fraction of the $A_{ \pm \pm}$component contribution in $B_{c}^{+} \rightarrow$ $J / \psi D_{s}^{*+}$ decay is calculated from the $f_{ \pm \pm}$value quoted in Table 1 by applying a correction to account for the different acceptances for the two component contributions:

$$
\Gamma_{ \pm \pm} / \Gamma=f_{ \pm \pm} \times \frac{\mathcal{A}_{B_{c}^{+} \rightarrow J / \psi D_{s}^{*+}}}{\mathcal{A}_{B_{c}^{+} \rightarrow J / \psi D_{s}^{*+}, \mathcal{A}_{ \pm \pm}}} .
$$

\section{Systematic uncertainties}

The systematic uncertainties of the measured values are determined by varying the analysis procedure and repeating all calculations. Although some sources can have rather large effects on the individual decay rate measurements, they largely cancel in the ratios of the branching fractions due to correlation between the effects on the different decay modes. The following groups of systematic uncertainties are considered.

The first group of sources of systematic uncertainty relates to possible differences between the data and simulation affecting the acceptances for the decay modes. Thus, an effect of the $B_{c}^{+}$production model is evaluated by varying the simulated $p_{\mathrm{T}}$ and $|\eta|$ spectra while preserving agreement with the data distributions obtained using the abundant $B_{c}^{+} \rightarrow J / \psi \pi^{+}$channel. These variations have very similar effects on the acceptances for the different decay modes, thus giving rather moderate estimates of the uncertainties, not exceeding $3 \%$ in total, on the ratios of branching fractions. The effect of presence of other $D_{s}^{+}$decay modes with $\left(K^{+} K^{-} \pi^{+}\right)$final state on the calculated acceptances is studied with a separate $\mathrm{MC}$ simulation. Its conservative estimate yields $0.4 \%$ which is assigned as $\mathcal{R}_{D_{s}^{+} / \pi^{+}}$and $\mathcal{R}_{D_{s}^{*+} / \pi^{+}}$ uncertainties. An uncertainty on the tracking efficiency is dominated by the uncertainty of the detector material description in the MC simulation. Samples generated with distorted geometries and with increased material are used to estimate the effect on track reconstruction efficiencies. When propagated to the ratios of branching fractions, these estimates give $0.5 \%$ uncertainty for $\mathcal{R}_{D_{s}^{+} / \pi^{+}}$and $\mathcal{R}_{D_{s}^{*+} / \pi^{+}}$due to the two extra tracks in $B_{c}^{+} \rightarrow J / \psi D_{s}^{(*)+}$ modes. Limited knowledge of the $B_{c}^{+}$and $D_{s}^{+}$lifetimes leads to an additional systematic uncertainty. The simulated proper decay times are varied within one standard deviation from the world average values [22] resulting in uncertainties of $\sim 1 \%$ assigned to $\mathcal{R}_{D_{s}^{+} / \pi^{+}}$and $\mathcal{R}_{D_{s}^{*+} / \pi^{+}}$due to the $B_{c}^{+}$lifetime, and $0.3 \%$ due to the $D_{s}^{+}$lifetime. Removing the requirement on $p_{\mathrm{T}}\left(B_{c}^{+}\right) / \sum p_{\mathrm{T}}(\mathrm{trk})$ is found to produce no noticeable effect on the measured values.

The next group of uncertainties originates from the signal extraction procedure. These uncertainties are evaluated separately for $J / \psi D_{s}^{+}$and $J / \psi \pi^{+}$candidate fits. For the former, the following variations of the fit model are applied and the difference is treated as a systematic uncertainty:

- different background mass shape parametrisations (threeparameter exponential, second- and third-order polynomials), different fitted mass range (reduced by up to $40 \mathrm{MeV}$ from each side independently);

- a double Gaussian or double-sided Crystal Ball function [27-29] for $B_{c}^{+} \rightarrow J / \psi D_{s}^{+}$signal description; variation of the modified Gaussian width within $10 \%$ of the MC simulation value;

- variation of the smoothness of the $B_{c}^{+} \rightarrow J / \psi D_{s}^{*+}$ signal mass templates, which is controlled by a parameter of the kernel estimation procedure [25];

- similar variation of the smoothness of the $B_{c}^{+} \rightarrow$ $J / \psi D_{s}^{(*)+}$ signal angular templates;

- variation of the smoothness of the sideband templates used for the background angular PDF construction; different ranges of the sidebands; different sideband interpolation procedure;

- modelling of the correlation between the mass and angular parts of the signal PDFs. This correlation takes place only at the detector level and manifests itself in degradation of the mass resolution for higher values of $\left|\cos \theta^{\prime}\left(\mu^{+}\right)\right|$. A dedicated fit model accounting for this effect is used for the data fit. The impact on the result is found to be negligible compared to the total uncertainty.

The first two items give the dominant contributions to the uncertainties of the ratios of branching fractions while the transverse polarisation fraction measurement is mostly affected by the background angular modelling variations. For the normalisation channel fit model, the similar variations of the background and signal mass shape parametrisation are applied. The deviations produced by the variations of the fits reach values as high as 10-15\% thus making them the dominant sources of systematic uncertainty.

The branching fractions of $D_{s}^{*+}$ [22] are varied in simulation within their uncertainties to estimate their effect on the measured quantities. Very small uncertainties are obtained 
Table 4 Relative systematic uncertainties on the measured ratios of branching fractions $R_{D_{s}^{+} / \pi^{+}}, R_{D_{s}^{*+} / \pi^{+}}, R_{D_{s}^{*+} / D_{s}^{+}}$ and on the transverse polarisation fraction $\Gamma_{ \pm \pm} / \Gamma$

\begin{tabular}{lcccc}
\hline Source & \multicolumn{2}{l}{ Uncertainty $(\%)$} & & \\
\cline { 2 - 5 } & $R_{D_{s}^{+} / \pi^{+}}$ & $R_{D_{s}^{*+} / \pi^{+}}$ & $R_{D_{s}^{*+} / D_{s}^{+}}$ & $\Gamma_{ \pm \pm} / \Gamma$ \\
\hline Simulated $p_{\mathrm{T}}\left(B_{c}^{+}\right)$spectrum & 0.4 & 0.9 & 0.5 & 0.4 \\
Simulated $\left|\eta\left(B_{c}^{+}\right)\right|$spectrum & 1.9 & 2.4 & 0.6 & 0.2 \\
Other $D_{s}^{+}$decay modes contribution & 0.4 & 0.4 & - & - \\
Tracking efficiency & 0.5 & 0.5 & $<0.1$ & $<0.1$ \\
$B_{c}^{+}$lifetime & 1.2 & 1.3 & $<0.1$ & $<0.1$ \\
$D_{s}^{+}$lifetime & 0.3 & 0.3 & $<0.1$ & $<0.1$ \\
$B_{c}^{+} \rightarrow J / \psi D_{s}^{(*)+}$ signal extraction & 4.4 & 10.5 & 10.7 & 17.4 \\
$B_{c}^{+} \rightarrow J / \psi \pi^{+}$signal extraction & 8.5 & 8.5 & - & - \\
$D_{s}^{*+}$ branching fractions & $<0.1$ & $<0.1$ & $<0.1$ & 1.1 \\
MC sample sizes & 2.3 & 2.4 & 2.7 & 2.2 \\
\hline Total & 10.1 & 14.0 & 11.0 & 17.6 \\
\hline $\mathcal{B}_{D_{s}^{+} \rightarrow \phi\left(K^{+} K^{-}\right) \pi^{+}}$ & 5.9 & 5.9 & - & - \\
\hline
\end{tabular}

for the $\mathcal{R}_{D_{s}^{*+} / \pi^{+}}$and $\mathcal{R}_{D_{s}^{*+} / D_{s}^{+}}$, while for $\Gamma_{ \pm \pm} / \Gamma$, the estimate is $\sim 1 \%$.

The statistical uncertainties on the acceptance values due to the MC sample sizes are also treated as a separate source of systematic uncertainty and estimated to be 2-3\%.

In order to check for a possible bias from using three-muon triggers, vetoing the $D_{s}^{+}$meson daughter tracks identified as muons is tested and found not to affect the measurement.

Finally, since $\mathcal{B}_{D_{s}^{+} \rightarrow \phi\left(K^{+} K^{-}\right) \pi^{+}}$enters Eq. (2), its uncertainty, evaluated from Ref. [26] as $5.9 \%$, is propagated to the final values of the relative branching fractions.

The systematic uncertainties on the measured quantities are summarised in Table 4.

\section{Results}

The following ratios of the branching fractions are measured:

$$
\begin{aligned}
\mathcal{R}_{D_{s}^{+} / \pi^{+}} & =\frac{\mathcal{B}_{B_{c}^{+} \rightarrow J / \psi D_{s}^{+}}}{\mathcal{B}_{B_{c}^{+} \rightarrow J / \psi \pi^{+}}} \\
& =3.8 \pm 1.1 \text { (stat.) } \pm 0.4 \text { (syst.) } \pm 0.2(\mathrm{BF}) \\
\mathcal{R}_{D_{s}^{*+} / \pi^{+}} & =\frac{\mathcal{B}_{B_{c}^{+} \rightarrow J / \psi D_{s}^{*+}}}{\mathcal{B}_{B_{c}^{+} \rightarrow J / \psi \pi^{+}}} \\
& =10.4 \pm 3.1 \text { (stat.) } \pm 1.5 \text { (syst.) } \pm 0.6(\mathrm{BF}), \\
\mathcal{R}_{D_{s}^{*+} / D_{s}^{+}} & =\frac{\mathcal{B}_{B_{c}^{+} \rightarrow J / \psi D_{s}^{*+}}}{\mathcal{B}_{B_{c}^{+} \rightarrow J / \psi D_{s}^{+}}} \\
& =2.8_{-0.8}^{+1.2}(\text { stat. }) \pm 0.3 \text { (syst.) },
\end{aligned}
$$

where the BF uncertainty corresponds to the knowledge of $\mathcal{B}_{D_{s}^{+} \rightarrow \phi\left(K^{+} K^{-}\right) \pi^{+}}$. The relative contribution of the $A_{ \pm \pm}$component in $B_{c}^{+} \rightarrow J / \psi D_{S}^{*+}$ decay is measured to be
$\Gamma_{ \pm \pm} / \Gamma=0.38 \pm 0.23$ (stat.) \pm 0.07 (syst.)

These results are compared with those of the $\mathrm{LHCb}$ measurement [10] and to the expectations from various theoretical calculations in Table 5 and Fig. 7. The measurement agrees with the $\mathrm{LHCb}$ result. All ratios are well described by the recent perturbative QCD predictions [8]. The expectations from models in Refs. [3,5,7] as well as the sum-rules prediction [4] for the ratio $\mathcal{R}_{D_{s}^{*+} / D_{s}^{+}}$are consistent with the measurement. The QCD relativistic potential model predictions [3] are consistent with the measured $\mathcal{R}_{D_{s}^{+} / \pi^{+}}$ratio while the expectations from the sum rules [4] and models in Refs. [5-7] are somewhat smaller than the measured value. The predictions in Refs. [3-5,7] are also generally smaller than the measured ratio $\mathcal{R}_{D_{s}^{*+} / \pi^{+}}$; however, the discrepancies do not exceed two standard deviations when taking into account only the experimental uncertainty.

The measured fraction of the $A_{ \pm \pm}$component agrees well with the prediction of the relativistic independent quark model [9] and perturbative QCD [8].

\section{Conclusion}

A study of $B_{c}^{+} \rightarrow J / \psi D_{s}^{+}$and $B_{c}^{+} \rightarrow J / \psi D_{s}^{*+}$ decays has been performed. The ratios of the branching fractions $\mathcal{B}_{B_{c}^{+} \rightarrow J / \psi D_{s}^{+}} / \mathcal{B}_{B_{c}^{+} \rightarrow J / \psi \pi^{+}}, \quad \mathcal{B}_{B_{c}^{+} \rightarrow J / \psi D_{s}^{*+}} / \mathcal{B}_{B_{c}^{+} \rightarrow J / \psi \pi^{+}}$, $\mathcal{B}_{B_{c}^{+} \rightarrow J / \psi D_{s}^{*+}} / \mathcal{B}_{B_{c}^{+} \rightarrow J / \psi D_{s}^{+}}$and the transverse polarisation fraction of $B_{c}^{+} \rightarrow J / \psi D_{s}^{*+}$ decay have been measured by the ATLAS experiment at the LHC using $p p$ collision data corresponding to an integrated luminosity of $4.9 \mathrm{fb}^{-1}$ at $7 \mathrm{TeV}$ centre-of-mass energy and $20.6 \mathrm{fb}^{-1}$ at $8 \mathrm{TeV}$. The polarisation is found to be well described by the available 
Table 5 Comparison of the results of this measurement with those of $\mathrm{LHCb}[10]$ and theoretical predictions based on a QCD relativistic potential model [3], QCD sum rules [4], relativistic constituent quark model (RCQM) [5], BSW relativistic quark model (with fixed average transverse quark momentum $\omega=0.40 \mathrm{GeV}$ ) [6], light-front quark model (LFQM) [7], perturbative QCD (pQCD) [8], and relativistic independent quark model (RIQM) [9]. The uncertainties of the theoretical predictions are shown if they are explicitly quoted in the corresponding papers. Statistical and systematic uncertainties added in quadrature are shown for the results of ATLAS and LHCb

\begin{tabular}{ccccc}
\hline $\mathcal{R}_{D_{s}^{+} / \pi^{+}}$ & $\mathcal{R}_{D_{s}^{*+} / \pi^{+}}$ & $\mathcal{R}_{D_{s}^{*+} / D_{s}^{+}}$ & $\Gamma_{ \pm \pm} / \Gamma$ & Ref. \\
\hline $3.8 \pm 1.2$ & $10.4 \pm 3.5$ & $2.8_{-0.9}^{+1.2}$ & $0.38 \pm 0.24$ & ATLAS \\
$2.90 \pm 0.62$ & - & $2.37 \pm 0.57$ & $0.52 \pm 0.20$ & LHCb [10] \\
\hline 2.6 & 4.5 & 1.7 & - & QCD potential model [3] \\
1.3 & 5.2 & 3.9 & - & QCD sum rules [4] \\
2.0 & 5.7 & 2.9 & - & RCQM [5] \\
2.2 & - & - & - & BSW [6] \\
$2.06 \pm 0.86$ & - & $3.01 \pm 1.23$ & LFQM [7] \\
$3.45_{-0.17}^{+0.49}$ & - & $2.54_{-0.21}^{+0.07}$ & $0.48 \pm 0.04$ & pQCD [8] \\
- & - & - & 0.410 & RIQM [9] \\
\hline
\end{tabular}

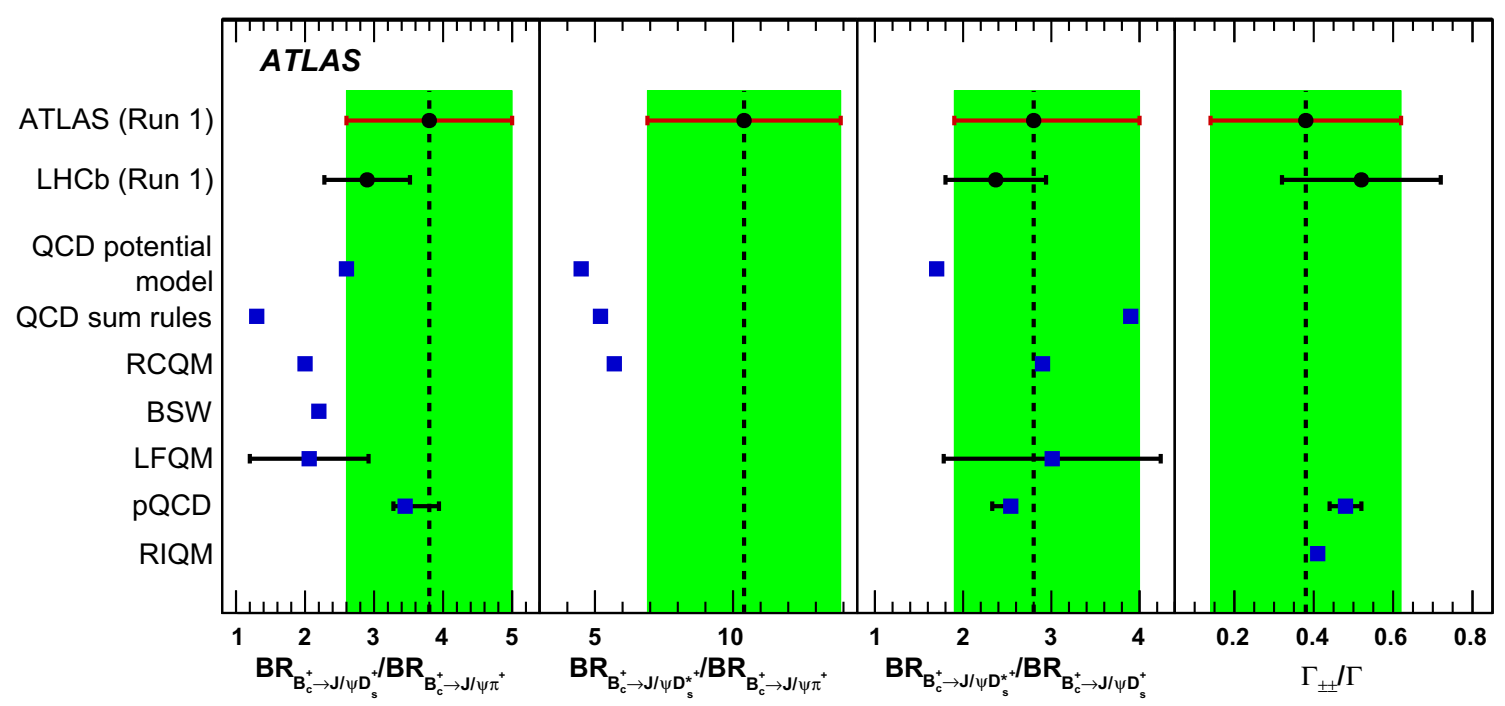

Fig. 7 Comparison of the results of this measurement with those of LHCb [10] and theoretical predictions based on a QCD relativistic potential model [3], QCD sum rules [4], relativistic constituent quark model (RCQM) [5], BSW relativistic quark model (with fixed average transverse quark momentum $\omega=0.40 \mathrm{GeV}$ ) [6], light-front quark model (LFQM) [7], perturbative QCD (pQCD) [8], and relativistic independent quark model (RIQM) [9]. The uncertainties of the theoretical predictions are shown if they are explicitly quoted in the corresponding papers. Statistical and systematic uncertainties added in quadrature are quoted for the results of ATLAS and LHCb. theoretical approaches. The measured ratios of the branching fraction are generally described by perturbative QCD, sum rules, and relativistic quark models. There is an indication of underestimation of the decay rates for the $B_{c}^{+} \rightarrow J / \psi D_{s}^{(*)+}$ decays by some models, although the discrepancies do not exceed two standard deviations when taking into account only the experimental uncertainty. The measurement results agree with those published by the LHCb experiment.

Acknowledgments We thank CERN for the very successful operation of the LHC, as well as the support staff from our institutions without whom ATLAS could not be operated efficiently. We acknowledge the support of ANPCyT, Argentina; YerPhI, Armenia; ARC,
Australia; BMWFW and FWF, Austria; ANAS, Azerbaijan; SSTC, Belarus; CNPq and FAPESP, Brazil; NSERC, NRC and CFI, Canada; CERN; CONICYT, Chile; CAS, MOST and NSFC, China; COLCIENCIAS, Colombia; MSMT CR, MPO CR and VSC CR, Czech Republic; DNRF, DNSRC and Lundbeck Foundation, Denmark; EPLANET, ERC and NSRF, European Union; IN2P3-CNRS, CEA-DSM/IRFU, France; GNSF, Georgia; BMBF, DFG, HGF, MPG and AvH Foundation, Germany; GSRT and NSRF, Greece; RGC, Hong Kong SAR, China; ISF, MINERVA, GIF, I-CORE and Benoziyo Center, Israel; INFN, Italy; MEXT and JSPS, Japan; CNRST, Morocco; FOM and NWO, Netherlands; BRF and RCN, Norway; MNiSW and NCN, Poland; GRICES and FCT, Portugal; MNE/IFA, Romania; MES of Russia and NRC KI, Russian Federation; JINR; MSTD, Serbia; MSSR, Slovakia; ARRS and MIZŠ, Slovenia; DST/NRF, South Africa; MINECO, Spain; SRC and Wallenberg Foundation, Sweden; SER, SNSF and Cantons of Bern and 
Geneva, Switzerland; NSC, Taiwan; TAEK, Turkey; STFC, the Royal Society and Leverhulme Trust, United Kingdom; DOE and NSF, United States of America. The crucial computing support from all WLCG partners is acknowledged gratefully, in particular from CERN and the ATLAS Tier-1 facilities at TRIUMF (Canada), NDGF (Denmark, Norway, Sweden), CC-IN2P3 (France), KIT/GridKA (Germany), INFNCNAF (Italy), NL-T1 (Netherlands), PIC (Spain), ASGC (Taiwan), RAL (UK) and BNL (USA) and in the Tier-2 facilities worldwide.

Open Access This article is distributed under the terms of the Creative Commons Attribution 4.0 International License (http://creativecomm ons.org/licenses/by/4.0/), which permits unrestricted use, distribution, and reproduction in any medium, provided you give appropriate credit to the original author(s) and the source, provide a link to the Creative Commons license, and indicate if changes were made.

Funded by SCOAP ${ }^{3}$.

\section{References}

1. CDF Collaboration, F. Abe et al., Phys. Rev. Lett. 81, 2432-2437 (1998). arXiv:hep-ex/9805034. doi:10.1103/PhysRevLett.81.2432

2. ATLAS Collaboration, Phys. Rev. Lett. 113, 212004 (2014). arXiv:1407.1032 [hep-ex]. doi:10.1103/PhysRevLett.113.212004

3. P. Colangelo, F. De Fazio, Phys. Rev. D 61, 034012 (2000). doi:10. 1103/PhysRevD.61.034012. arXiv:hep-ph/9909423

4. V. Kiselev, Exclusive decays and lifetime of $B_{c}$ meson in QCD sum rules (2002). arXiv:hep-ph/0211021 [hep-ph]

5. M. Ivanov, J. Korner, P. Santorelli, Phys. Rev. D 73, 054024 (2006). doi:10.1103/PhysRevD.73.054024. arXiv:hep-ph/0602050

6. R. Dhir, R. Verma, Phys. Rev. D 79, 034004 (2009). doi:10.1103/ PhysRevD.79.034004. arXiv:0810.4284 [hep-ph]

7. H.-W. Ke, T. Liu, X.-Q. Li, Phys. Rev. D 89, 017501 (2014). doi:10. 1103/PhysRevD.89.017501. arXiv:1307.5925 [hep-ph]

8. Z. Rui, Z.-T. Zou, Phys. Rev. D 90, 114030 (2014). doi:10.1103/ PhysRevD.90.114030. arXiv:1407.5550 [hep-ph]

9. S. Kar et al., Phys. Rev. D 88, 094014 (2013). doi:10.1103/ PhysRevD.88.094014

10. LHCb Collaboration, R. Aaij et al., Phys. Rev. D 87, 112012 (2013). arXiv:1304.4530 [hep-ex]. doi:10.1103/PhysRevD.87.112012

11. ATLAS Collaboration, JINST 3, S08003 (2008). doi:10.1088/ $1748-0221 / 3 / 08 / \mathrm{S} 08003$
12. T. Sjostrand, S. Mrenna, P. Skands, JHEP 0605, 026 (2006). arXiv:hep-ph/0603175. doi:10.1088/1126-6708/2006/05/026

13. A. Berezhnoy, A. Likhoded and O. Yushchenko, Phys. Atom. Nucl. 59 (1996) 709-713, arXiv:hep-ph/9504302 [hep-ph]

14. A. Berezhnoy, V. Kiselev, A. Likhoded, Z. Phys. A 356, 79-87 (1996). arXiv:hep-ph/9602347. doi:10.1007/s002180050151

15. A. Berezhnoy et al., Phys. Atom. Nucl. 60 (1997) 1729-1740, arXiv:hep-ph/9703341 [hep-ph]

16. A. Berezhnoy, Phys. Atom. Nucl. 68, 1866-1872 (2005) arXiv:hep-ph/0407315. doi:10.1134/1.2131116

17. D. Lange, Nucl. Instrum. Meth. A 462, 152-155 (2001). doi:10. 1016/S0168-9002(01)00089-4

18. ATLAS Collaboration, Eur. Phys. J. C 70, 823-874 (2010). arXiv:1005.4568 [physics.ins-det]. doi:10.1140/epjc/ s10052-010-1429-9

19. GEANT4 Collaboration, S. Agostinelli et al., Nucl. Instrum. Meth. A 506, 250-303 (2003). doi:10.1016/S0168-9002(03)01368-8

20. J. Allison et al., IEEE Trans. Nucl. Sci. 53, 270 (2006). doi:10. 1109/TNS.2006.869826

21. V. Kostyukhin, VKalVrt - package for vertex reconstruction in ATLAS, ATL-PHYS-2003-031 (2003). http://cds.cern.ch/record/ 685551

22. K. A. Olive et al., Particle Data Group, Chin. Phys. C 38, 090001 (2014). doi:10.1088/1674-1137/38/9/090001

23. ZEUS Collaboration, S. Chekanov et al., Eur. Phys. J. C 44, 13-25 (2005). arXiv:hep-ex/0505008. doi:10.1140/epjc/s2005-02346-2

24. G. Aad et al., Nucl. Phys. B 864, 341-381 (2012). arXiv: 1206.3122 [hep-ex]. doi:10.1016/j.nuclphysb.2012.07.009

25. K. Cranmer, Comput. Phys. Commun. 136, 198-207 (2001). arXiv:hep-ex/0011057 [hep-ex]. doi:10.1016/ S0010-4655(00)00243-5

26. CLEO Collaboration, J. Alexander et al., Phys. Rev. Lett. 100, 161804 (2008). doi:10.1103/PhysRevLett.100.161804. arXiv:0801.0680 [hep-ex]

27. M. Oreglia, A study of the reactions $\psi^{\prime} \rightarrow \gamma \gamma \psi, \mathrm{PhD}$ thesis, Stanford University, SLAC-R-236 (1980). http://www.slac.stanford. edu/pubs/slacreports/slac-r-236.html

28. J. Gaiser, Charmonium spectroscopy from radiative decays of the $J / \psi$ and $\psi^{\prime}, \mathrm{PhD}$ thesis, Stanford University, SLAC-R-255 (1982). http://www.slac.stanford.edu/pubs/slacreports/slac-r-255.html

29. T. Skwarnicki, A study of the radiative cascade transitions between the $\Upsilon^{\prime}$ and $\Upsilon$ resonances, PhD thesis, Institute of Nuclear Physics, Krakow, DESY-F31-86-02 (1986). http://inspirehep.net/record/ 230779/ 


\section{ATLAS Collaboration}

G. Aad ${ }^{85}$, B. Abbott ${ }^{113}$, J. Abdallah ${ }^{151}$, O. Abdinov ${ }^{11}$, R. Aben ${ }^{107}$, M. Abolins ${ }^{90}$, O. S. AbouZeid ${ }^{158}$, H. Abramowicz ${ }^{153}$, H. Abreu ${ }^{152}$, R. Abreu ${ }^{30}$, Y. Abulaiti ${ }^{146 a, 146 b}$, B. S. Acharya ${ }^{164 a, 164 b, a}$, L. Adamczyk ${ }^{38 a}$, D. L. Adams ${ }^{25}$, J. Adelman ${ }^{108}$, S. Adomeit ${ }^{100}$, T. Adye ${ }^{131}$, A. A. Affolder ${ }^{74}$, T. Agatonovic-Jovin ${ }^{13}$, J. A. Aguilar-Saavedra ${ }^{126 a, 126 f}$, S. P. Ahlen ${ }^{22}$,

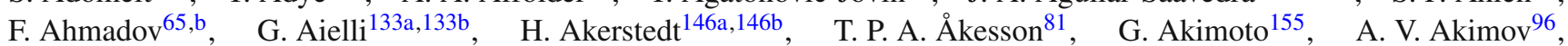
G. L. Alberghi ${ }^{20 a, 20 b}$, J. Albert ${ }^{169}$, S. Albrand ${ }^{55}$, M. J. Alconada Verzini ${ }^{71}$, M. Aleksa ${ }^{30}$, I. N. Aleksandrov ${ }^{65}$, $\begin{array}{llll}\text { C. Alexa } & \text { 26a, } & \text { G. Alexander } & 153\end{array}$ T. Alexopoulos ${ }^{10}, \quad$ M. Alhroob ${ }^{113}, \quad$ G. Alimonti ${ }^{91 a}, \quad$ L. Alio ${ }^{85}$, J. Alison ${ }^{31}$, S. P. Alkire ${ }^{35}$, B. M. M. Allbrooke ${ }^{18}$, P. P. Allport ${ }^{74}$, A. Aloisio ${ }^{104 a, 104 b}$, A. Alonso ${ }^{36}$, F. Alonso ${ }^{71}$, C. Alpigiani ${ }^{76}$, A. Altheimer ${ }^{35}$, B. Alvarez Gonzalez ${ }^{30}$, D. Álvarez Piqueras ${ }^{167}$, M. G. Alviggi 104a,104b, B. T. Amadio ${ }^{15}$, K. Amako ${ }^{66}$, Y. Amaral Coutinho ${ }^{24 a}$, C. Amelung ${ }^{23}$, D. Amidei ${ }^{89}$, S. P. Amor Dos Santos ${ }^{126 a, 126 c}$, A. Amorim ${ }^{126 a, 126 b}$, S. Amoroso ${ }^{48}$, N. $A_{m r a m}^{153}$, G. Amundsen $^{23}$, C. Anastopoulos ${ }^{139}$, L. S. Ancu ${ }^{49}$, N. Andari ${ }^{30}$, T. Andeen ${ }^{35}$, C. F. Anders ${ }^{58 b}$, G. Anders $^{30}$, J. K. Anders ${ }^{74}$, K. J. Anderson ${ }^{31}$, A. Andreazza ${ }^{91 a, 91 b}$, V. Andrei ${ }^{58 a}$, S. Angelidakis ${ }^{9}$, I. Angelozzi ${ }^{107}$, P. Anger ${ }^{44}$, A. Angerami ${ }^{35}$, F. Anghinolfi ${ }^{30}$, A. V. Anisenkov ${ }^{109, c}$, N. Anjos ${ }^{12}$, A. Annovi ${ }^{124 a, 124 b}$, M. Antonellii ${ }^{47}$, A. Antonov ${ }^{98}$, J. Antos ${ }^{144 b}$, F. Anulli ${ }^{132 a}$, M. Aoki ${ }^{66}$, L. Aperio Bella ${ }^{18}$, G. Arabidze ${ }^{90}$, Y. Arai ${ }^{66}$, J. P. Araque ${ }^{126 a}$,

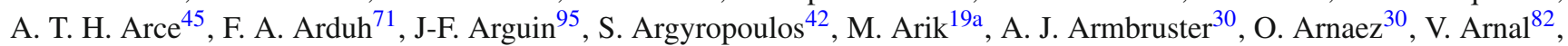
H. Arnold ${ }^{48}$, M. Arratia ${ }^{28}$, O. Arslan ${ }^{21}$, A. Artamonov ${ }^{97}$, G. Artoni ${ }^{23}$, S. Asai ${ }^{155}$, N. Asbah ${ }^{42}$, A. Ashkenazi ${ }^{153}$, B. Åsman 146a,146b, L. Asquith ${ }^{149}$, K. Assamagan ${ }^{25}$, R. Astalos ${ }^{144 a}$, M. Atkinson ${ }^{165}$, N. B. Atlay ${ }^{141}$, B. Auerbach ${ }^{6}$, K. Augsten ${ }^{128}$, M. Aurousseau ${ }^{145 b}$, G. Avolio ${ }^{30}$, B. Axen ${ }^{15}$, M. K. Ayoub ${ }^{117}$, G. Azuelos ${ }^{95, d}$, M. A. Baak ${ }^{30}$, A. E. Baas ${ }^{58 a}$, C. Bacci ${ }^{134 a, 134 b}$, H. Bachacou ${ }^{136}$, K. Bachas ${ }^{154}$, M. Backes ${ }^{30}$, M. Backhaus ${ }^{30}$, P. Bagiacchi ${ }^{132 a, 132 b}$, P. Bagnaia ${ }^{132 a, 132 b}$, Y. Bai ${ }^{33 a}$, T. Bain ${ }^{35}$, J. T. Baines ${ }^{131}$, O. K. Bakerr ${ }^{176}$, P. Balek ${ }^{129}$, T. Balestri ${ }^{148}$, F. Balli ${ }^{84}$, E. Banas ${ }^{39}$, Sw. Banerjee ${ }^{173}$, A. A. E. Bannoura ${ }^{175}$, H. S. Bansil ${ }^{18}$, L. Barak ${ }^{30}$, E. L. Barberio ${ }^{88}$, D. Barberis ${ }^{50 a, 50 b}$, M. Barbero ${ }^{85}$, T. Barillari ${ }^{101}$, M. Barisonzi ${ }^{164 a, 164 b}$, T. Barklow ${ }^{143}$, N. Barlow ${ }^{28}$, S. L. Barnes ${ }^{84}$, B. M. Barnett ${ }^{131}$, R. M. Barnett ${ }^{15}$, Z. Barnovska ${ }^{5}$, A. Baroncelli ${ }^{134 a}$, G. Barone ${ }^{49}$, A. J. Barr ${ }^{120}$, F. Barreiro ${ }^{82}$, J. Barreiro Guimarães da Costa ${ }^{57}$, R. Bartoldus ${ }^{143}$, A. E. Barton ${ }^{72}$, P. Bartos ${ }^{144}$, A. Basalaev ${ }^{123}$, A. Bassalat ${ }^{17}$, A. Basye ${ }^{165}$, R. L. Bates ${ }^{53}$, S. J. Batista ${ }^{158}$, J. R. Batley ${ }^{28}$,

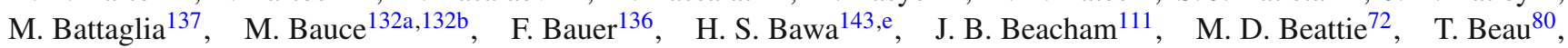
P. H. Beauchemin ${ }^{161}$, R. Beccherle ${ }^{124 a, 124 b}$, P. Bechtle ${ }^{21}$, H. P. Beck ${ }^{17, f}$, K. Becker ${ }^{120}$, M. Becker ${ }^{83}$, S. Becker ${ }^{100}$, M. Beckingham ${ }^{170}$, C. Becot $^{117}$, A. J. Beddall ${ }^{19 b}$, A. Beddall ${ }^{19 b}$, V. A. Bednyakov ${ }^{65}$, C. P. Bee ${ }^{148}$, L. J. Beemster ${ }^{107}$, T. A. Beermann ${ }^{175}$, M. Begel ${ }^{25}$, J. K. Behr ${ }^{120}$, C. Belanger-Champagne ${ }^{87}$, W. H. Bell ${ }^{49}$, G. Bella ${ }^{153}$, L. Bellagamba ${ }^{20 a}$, A. Bellerive ${ }^{29}$, M. Bellomo ${ }^{86}$, K. Belotskiy ${ }^{98}$, O. Beltramello ${ }^{30}$, O. Benary ${ }^{153}$, D. Benchekroun ${ }^{135 a}$, M. Bender ${ }^{100}$, K. Bendtz ${ }^{146 a, 146 b}$, N. Benekos ${ }^{10}$, Y. Benhammou ${ }^{153}$, E. Benhar Noccioli49, J. A. Benitez Garcia ${ }^{159 b}$, D. P. Benjamin ${ }^{45}$, J. R. Bensinger ${ }^{23}$, S. Bentvelsen ${ }^{107}$, L. Beresford ${ }^{120}$, M. Beretta ${ }^{47}$, D. Berge ${ }^{107}$, E. Bergeaas Kuutmann ${ }^{166}$, N. Berger ${ }^{5}$, F. Berghaus ${ }^{169}$, J. Beringer ${ }^{15}$, C. Bernard ${ }^{22}$, N. R. Bernard ${ }^{86}$, C. Bernius ${ }^{110}$, F. U. Bernlochner ${ }^{21}$, T. Berry ${ }^{77}$, P. Berta ${ }^{129}$, C. Bertella ${ }^{83}$, G. Bertoli146a,146b , F. Bertolucci ${ }^{124 a, 124 b}$, C. Bertsche ${ }^{113}$, D. Bertsche ${ }^{113}$, M. I. Besana ${ }^{91 a}$, G. J. Besjes ${ }^{106}$, O. Bessidskaia Bylund ${ }^{146 a, 146 b}$, M. Bessner ${ }^{42}$, N. Besson ${ }^{136}$, C. Betancourt ${ }^{48}$, S. Bethke ${ }^{101}$, A. J. Bevan ${ }^{76}$, W. Bhimji ${ }^{46}$, R. M. Bianchi ${ }^{125}$, L. Bianchini ${ }^{23}$, M. Bianco ${ }^{30}$, O. Biebel ${ }^{100}$, S. P. Bieniek ${ }^{78}$, M. Biglietti ${ }^{134 a}$, J. Bilbao De Mendizabal ${ }^{49}$, H. Bilokon ${ }^{47}$, M. Bindi ${ }^{54}$, S. Binet ${ }^{117}$, A. Bingul ${ }^{19 b}$, C. Bini ${ }^{132 a, 132 b}$, C. W. Black ${ }^{150}$, J. E. Black ${ }^{143}$, K. M. Black ${ }^{22}$, D. Blackburn ${ }^{138}$, R. E. Blair ${ }^{6}$, J.-B. Blanchard ${ }^{136}$, J. E. Blanco ${ }^{77}$, T. Blazek ${ }^{144 a}$, I. Bloch ${ }^{42}$, C. Blocker ${ }^{23}$, W. Blum ${ }^{83, *}$, U. Blumenschein $^{54}$, G. J. Bobbink ${ }^{107}$, V. S. Bobrovnikov ${ }^{109, c}$, S. S. Bocchetta ${ }^{81}$, A. Bocci4 ${ }^{45}$, C. Bock ${ }^{100}$, M. Boehler ${ }^{48}$,

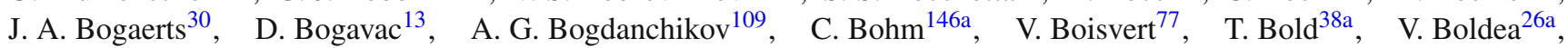
A. S. Boldyrev ${ }^{99}$, M. Bomben ${ }^{80}$, M. Bona ${ }^{76}$, M. Boonekamp ${ }^{136}$, A. Borisov ${ }^{130}$, G. Borissov ${ }^{72}$, S. Borroni ${ }^{42}$, J. Bortfeldt ${ }^{100}$, V. Bortolotto ${ }^{60 a, 60 b, 60 c}$, K. Bos ${ }^{107}$, D. Boscherini20a , M. Bosman ${ }^{12}$, J. Boudreau ${ }^{125}$, J. Bouffard ${ }^{2}$, E. V. Bouhova-Thacker ${ }^{72}$, D. Boumediene ${ }^{34}$, C. Bourdarios ${ }^{117}$, N. Bousson ${ }^{114}$, A. Boveia ${ }^{30}$, J. Boyd ${ }^{30}$, I. R. Boyko ${ }^{65}$, I. Bozic ${ }^{13}$, J. Bracinik ${ }^{18}$, A. Brandt ${ }^{8}$, G. Brandt ${ }^{54}$, O. Brandt ${ }^{58 a}$, U. Bratzler ${ }^{156}$, B. Brau ${ }^{86}$, J. E. Brau ${ }^{116}$, H. M. Braun ${ }^{175, *}$, S. F. Brazzale ${ }^{164 a, 164 c}$, W. D. Breaden Madden ${ }^{53}$, K. Brendlinger ${ }^{122}$, A. J. Brennan ${ }^{88}$, L. Brenner ${ }^{107}$, R. Brenner ${ }^{166}$, S. Bressler ${ }^{172}$, K. Bristow ${ }^{145 c}$, T. M. Bristow ${ }^{46}$, D. Britton ${ }^{53}$, D. Britzger ${ }^{42}$, F. M. Brochu ${ }^{28}$, I. Brock ${ }^{21}$, R. Brock ${ }^{90}$, J. Bronner ${ }^{101}$, G. Brooijmans ${ }^{35}$, T. Brooks ${ }^{77}$, W. K. Brooks ${ }^{32 b}$, J. Brosamer ${ }^{15}$, E. Brost ${ }^{116}$, J. Brown ${ }^{55}$, P. A. Bruckman de Renstrom ${ }^{39}$, D. Bruncko ${ }^{144 b}$, R. Bruneliere ${ }^{48}$, A. Bruni ${ }^{20 a}$, G. Bruni ${ }^{20 a}$, M. Bruschi ${ }^{20 a}$, N. Bruscino ${ }^{21}$, L. Bryngemark ${ }^{81}$, T. Buanes ${ }^{14}$, Q. Buat ${ }^{142}$,

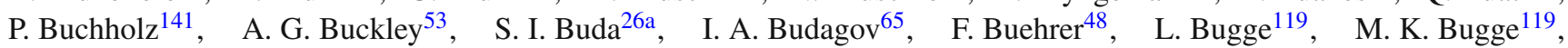
O. Bulekov ${ }^{98}$, D. Bullock ${ }^{8}$, H. Burckhart ${ }^{30}$, S. Burdin ${ }^{74}$, B. Burghgrave ${ }^{108}$, S. Burke ${ }^{131}$, I. Burmeister ${ }^{43}$, E. Busato ${ }^{34}$, D. Büscher ${ }^{48}$, V. Büscher ${ }^{83}$, P. Bussey ${ }^{53}$, J. M. Butler ${ }^{22}$, A. I. Butt ${ }^{3}$, C. M. Buttar ${ }^{53}$, J. M. Butterworth ${ }^{78}$, P. Butti ${ }^{107}$, W. Buttinger ${ }^{25}$, A. Buzatu ${ }^{53}$, A. R. Buzykaev ${ }^{109, c}$, S. Cabrera Urbán ${ }^{167}$, D. Caforio ${ }^{128}$, V. M. Cairo ${ }^{37 a, 37 b}$, O. Cakir ${ }^{4 a}$, P. Calafiura ${ }^{15}$, A. Calandri ${ }^{136}$, G. Calderini ${ }^{80}$, P. Calfayan ${ }^{100}$, L. P. Caloba ${ }^{24 a}$, D. Calvet ${ }^{34}$, S. Calvet ${ }^{34}$, R. Camacho Toro ${ }^{31}$, 


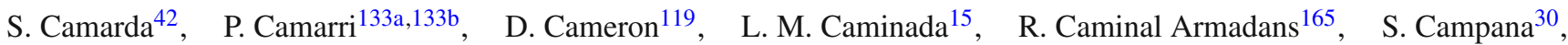
M. Campanelli ${ }^{78}$, A. Campoverde ${ }^{148}$, V. Canale ${ }^{104 a, 104 b}$, A. Canepa ${ }^{159 a}$, M. Cano Bret ${ }^{76}$, J. Cantero ${ }^{82}$, R. Cantrill ${ }^{126 a}$, T. Cao ${ }^{40}$, M. D. M. Capeans Garrido ${ }^{30}$, I. Caprini ${ }^{26 a}$, M. Caprini ${ }^{26 a}$, M. Capua ${ }^{37 a, 37 b}$, R. Caputo ${ }^{83}$, R. Cardarelli ${ }^{133 a}$, F. Cardillo ${ }^{48}$, T. Carli $^{30}$ ， G. Carlino ${ }^{104 a}$, L. Carminati ${ }^{91 a, 91 b}$, S. Caron ${ }^{106}$, E. Carquin ${ }^{32 a}$, G. D. Carrillo-Montoya ${ }^{8}$, J. R. Carter ${ }^{28}$, J. Carvalho ${ }^{126 a, 126 c}$, D. Casadei ${ }^{78}$, M. P. Casado ${ }^{12}$, M. Casolino ${ }^{12}$, E. Castaneda-Miranda ${ }^{145 b}$, A. Castelli ${ }^{107}$, V. Castillo Gimenez ${ }^{167}$, N. F. Castro ${ }^{126 a, g}$, P. Catastini ${ }^{57}$, A. Catinaccio ${ }^{30}$, J. R. Catmore ${ }^{119}$, A. Cattai ${ }^{30}$, J. Caudron ${ }^{83}$, V. Cavaliere ${ }^{165}$, D. Cavalli ${ }^{91 a}$, M. Cavalli-Sforza ${ }^{12}$, V. Cavasinni ${ }^{124 a, 124 b}$, F. Ceradini ${ }^{134 a, 134 b}$, B. C. Cerio ${ }^{45}$, K. Cerny ${ }^{129}$, A. S. Cerqueira ${ }^{24 b}$, A. Cerri ${ }^{149}$, L. Cerrito ${ }^{76}$, F. Cerutti ${ }^{15}$, M. Cerv ${ }^{30}$, A. Cervelli17, S. A. Cetin ${ }^{19 c}$, A. Chafaq ${ }^{135 a}$, D. Chakraborty ${ }^{108}$, I. Chalupkova ${ }^{129}$, P. Chang ${ }^{165}$, B. Chapleau ${ }^{87}$, J. D. Chapman ${ }^{28}$, D. G. Charlton ${ }^{18}$, C. C. Chau ${ }^{158}$, C. A. Chavez Barajas ${ }^{149}$, S. Cheatham ${ }^{152}$, A. Chegwidden ${ }^{90}$, S. Chekanov' ${ }^{6}$ S. V. Chekulaev ${ }^{159 a}$, G. A. Chelkov ${ }^{65, h}$, M. A. Chelstowska ${ }^{89}$, C. Chen ${ }^{64}$, H. Chen ${ }^{25}$, K. Chen ${ }^{148}$, L. Chen ${ }^{33 d, i}$, S. Chen ${ }^{33 c}$, X. Chen ${ }^{33 f}$, Y. Chen ${ }^{67}$, H. C. Cheng ${ }^{89}$,

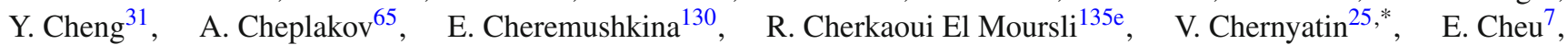
L. Chevalier ${ }^{136}$, V. Chiarella ${ }^{47}$, J. T. Childers ${ }^{6}$, G. Chiodini ${ }^{73 a}$, A. S. Chisholm ${ }^{18}$, R. T. Chislett $^{78}$, A. Chitan ${ }^{26 a}$,

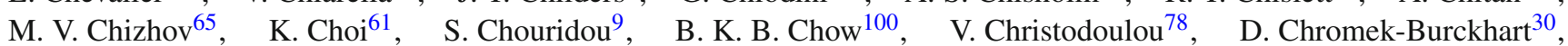
J. Chudoba ${ }^{127}$, A. J. Chuinard ${ }^{87}$, J. J. Chwastowski ${ }^{39}$, L. Chytka ${ }^{115}$, G. Ciapetti ${ }^{132 a, 132 b}$, A. K. Ciftci ${ }^{4 a}$, D. Cinca ${ }^{53}$, V. Cindro ${ }^{75}$, I. A. Cioara ${ }^{21}$, A. Ciocio ${ }^{15}$, Z. H. Citron ${ }^{172}$, M. Ciubancan ${ }^{26 a}$, A. Clark ${ }^{49}$, B. L. Clark ${ }^{57}$, P. J. Clark ${ }^{46}$, R. N. Clarke ${ }^{15}$, W. Cleland ${ }^{125}$, C. Clement ${ }^{146 a}$,146b, Y. Coadou ${ }^{85}$, M. Cobal ${ }^{164 a, 164 c}$, A. Coccaro ${ }^{138}$, J. Cochran ${ }^{64}$, L. Coffey ${ }^{23}$, J. G. Cogan $^{143}$, B. Cole ${ }^{35}$, S. Cole ${ }^{108}$, A. P. Colijn ${ }^{107}$, J. Collot ${ }^{55}$, T. Colombo ${ }^{58 c}$, G. Compostella ${ }^{101}$, P. Conde Muiño ${ }^{126 a, 126 b}$, E. Coniavitis ${ }^{48}$, S. H. Connell ${ }^{145 b}$, I. A. Connelly ${ }^{77}$, S. M. Consonni ${ }^{91 a}$,91b, V. Consorti ${ }^{48}$, S. Constantinescu ${ }^{26 a}$, C. Conta ${ }^{121 a, 121 b}$, G. Conti ${ }^{30}$, F. Conventi ${ }^{104 a, j}$, M. Cooke ${ }^{15}$, B. D. Cooper ${ }^{78}$, A. M. Cooper-Sarkar ${ }^{120}$, T. Cornelissen ${ }^{175}$, M. Corradi ${ }^{20 a}$, F. Corriveau ${ }^{87, \mathrm{k}}$, A. Corso-Radu ${ }^{163}, \quad$ A. Cortes-Gonzalez ${ }^{12}$, G. Cortiana ${ }^{101}$, G. Costa ${ }^{11 a}$, M. J. Costa ${ }^{167}$, D. Costanzo ${ }^{139}$, D. Côté ${ }^{8}, \quad$ G. Cottinn ${ }^{28}$, G. Cowan ${ }^{77}$, B. E. Cox ${ }^{84}, \quad$ K. Cranmer ${ }^{110}$, G. Cree $^{29}$, S. Crépé-Renaudin ${ }^{55}$ F. Crescioli ${ }^{80}$, W. A. Cribbs ${ }^{146 a, 146 b}, \quad$ M. Crispin Ortuzar ${ }^{120}$ M. Cristinziani ${ }^{21}$, V. Croft ${ }^{106}$, G. Crosetti ${ }^{37 a, 37 b}$, T. Cuhadar Donszelmann ${ }^{139}$, J. Cummings ${ }^{176}, \quad$ M. Curatolo ${ }^{47}, \quad$ C. Cuthbert ${ }^{150}$, H. Czirr ${ }^{141}$, P. Czodrowski ${ }^{3}$, S. D’Auria ${ }^{53}$, M. D'Onofrio ${ }^{74}$, M. J. Da Cunha Sargedas De Sousa ${ }^{126 a, 126 b}$, C. Da Via ${ }^{84}$, W. Dabrowski ${ }^{38 a}$, A. Dafinca ${ }^{120}$, T. Dai ${ }^{89}$, O. Dale ${ }^{14}$, F. Dallaire ${ }^{95}$, C. Dallapiccola ${ }^{86}$, M. Dam ${ }^{36}$, J. R. Dandoy ${ }^{31}$, N. P. Dang ${ }^{48}$, A. C. Daniells ${ }^{18}$, M. Danninger ${ }^{168}, \quad$ M. Dano Hoffmann ${ }^{136}, \quad$ V. Dao ${ }^{48}, \quad$ G. Darbo ${ }^{50 a}, \quad$ S. Darmora ${ }^{8}$, J. Dassoulas ${ }^{3}$, A. Dattagupta ${ }^{61}$, W. Davey ${ }^{21}$, C. David ${ }^{169}$, T. Davidek ${ }^{129}$, E. Davies ${ }^{120,1}$, M. Davies ${ }^{153}$, P. Davison ${ }^{78}$, Y. Davygora ${ }^{58 a}$, E. Dawe ${ }^{88}$, I. Dawson ${ }^{139}$, R. K. Daya-Ishmukhametova ${ }^{86}$, K. De $^{8}$, R. de Asmundis ${ }^{104 a}$, S. De Castro ${ }^{20 a, 20 b}$, S. De $\mathrm{Cecco}^{80}$, N. De Groot ${ }^{106}$, P. de Jong ${ }^{107}$, H. De la Torre ${ }^{82}$, F. De Lorenzi ${ }^{64}$, L. De Nooij ${ }^{107}$, D. De Pedis ${ }^{132 a}$, A. De Salvo ${ }^{132 a}$, U. De Sanctis ${ }^{149}$, A. De Santo ${ }^{149}$, J. B. De Vivie De Regie ${ }^{117}$, W. J. Dearnaley ${ }^{72}$, R. Debbe ${ }^{25}$, C. Debenedetti ${ }^{137}$, D. V. Dedovich ${ }^{65}$, I. Deigaard ${ }^{107}$, J. Del Peso ${ }^{82}$, T. Del Prete ${ }^{124 a, 124 b}$, D. Delgove ${ }^{117}$, F. Deliot ${ }^{136}$, C. M. Delitzsch ${ }^{49}$, M. Deliyergiyev ${ }^{75}$, A. Dell'Acqua ${ }^{30}$, L. Dell'Asta ${ }^{22}$, M. Dell'Orso ${ }^{124 a, 124 b}$, M. Della Pietra ${ }^{104 a, j}$, D. della Volpe ${ }^{49}$, M. Delmastro5, P. A. Delsart ${ }^{55}$, C. Deluca ${ }^{107}$, D. A. DeMarco ${ }^{158}$, S. Demers ${ }^{176}$, M. Demichev ${ }^{65}$, A. Demilly ${ }^{80}$, S. P. Denisov ${ }^{130}$, D. Derendarz ${ }^{39}$, J. E. Derkaoui ${ }^{135 d}$, F. Derue ${ }^{80}$, P. Dervan ${ }^{74}$, K. Desch ${ }^{21}$, C. Deterre ${ }^{42}$, P. O. Deviveiros ${ }^{30}$, A. Dewhurst ${ }^{131}$, S. Dhaliwal ${ }^{23}$, A. Di Ciaccio ${ }^{133 a, 133 b}$, L. Di Ciaccio ${ }^{5}$, A. Di Domenico ${ }^{132 a, 132 b}$, C. Di Donato 104a,104b, A. Di Girolamo ${ }^{30}$, B. Di Girolamo ${ }^{30}$, A. Di Mattia ${ }^{152}$, B. Di Micco ${ }^{134 a, 134 b}$, R. Di Nardo ${ }^{47}$, A. Di Simone ${ }^{48}$, R. Di Sipio ${ }^{158}$, D. Di Valentino ${ }^{29}, \quad$ C. Diaconu ${ }^{85}, \quad$ M. Diamond ${ }^{158}$, F. A. Dias ${ }^{46}, \quad$ M. A. Diaz ${ }^{32 a}$, E. B. Diehl ${ }^{89}$, J. Dietrich ${ }^{16}$, S. Diglio ${ }^{85}$, A. Dimitrievska ${ }^{13}$, J. Dingfelder ${ }^{21}$, P. Dita ${ }^{26 a}$, S. Dita $^{26 a}$, F. Dittus ${ }^{30}$, F. Djama ${ }^{85}$,

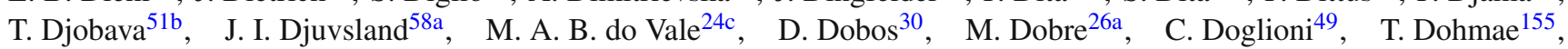
J. Dolejsi ${ }^{129}$, Z. Dolezal ${ }^{129}$, B. A. Dolgoshein ${ }^{98, *}$, M. Donadelli24d, S. Donati124a,124b, P. Dondero ${ }^{121 a, 121 b}$, J. Donini ${ }^{34}$, J. Dopke ${ }^{131}$, A. Doria ${ }^{104 a}$, M. T. Dova ${ }^{71}$, A. T. Doyle ${ }^{53}$, E. Drechsler ${ }^{54}$, M. Dris ${ }^{10}$, E. Dubreuil ${ }^{34}$, E. Duchovni ${ }^{172}$, G. Duckeck ${ }^{100}$, O. A. Ducu ${ }^{26 a, 85}$, D. Duda ${ }^{175}$, A. Dudarev ${ }^{30}$, L. Duflot ${ }^{117}$, L. Duguid ${ }^{77}$, M. Dührssen ${ }^{30}$, M. Dunford ${ }^{58 a}$, H. Duran Yildiz ${ }^{4 a}$, M. Düren ${ }^{52}$, A. Durglishvilis1b, D. Duschinger ${ }^{44}$, M. Dyndal ${ }^{38 a}$, C. Eckardt ${ }^{42}$, K. M. Ecker ${ }^{101}$, R. C. Edgar $^{89}$, W. Edson ${ }^{2}$, N.C. Edwards ${ }^{46}$, W. Ehrenfeld ${ }^{21}$, T. Eifert ${ }^{30}$, G. Eigen ${ }^{14}$, K. Einsweiler ${ }^{15}$, T. Ekelof ${ }^{166}$, M. El Kacimi ${ }^{135 c}$, M. Ellert ${ }^{166}$, S. Elles ${ }^{5}$, F. Ellinghaus ${ }^{83}$, A. A. Elliot ${ }^{169}$, N. Ellis ${ }^{30}$, J. Elmsheuser ${ }^{100}$, M. Elsing ${ }^{30}$, D. Emeliyanov ${ }^{131}$, Y. Enari ${ }^{155}$, O. C. Endner ${ }^{83}$, M. Endo ${ }^{118}$, J. Erdmann ${ }^{43}$, A. Ereditato ${ }^{17}$, G. Ernis ${ }^{175}$, J. Ernst ${ }^{2}$, M. Ernst $^{25}$, S. Errede ${ }^{165}$, E. Ertel ${ }^{83}$, M. Escalier ${ }^{117}, \quad$ H. Esch $^{43}, \quad$ C. Escobar ${ }^{125}$, B. Esposito ${ }^{47}$, A. I. Etienvre ${ }^{136}$,

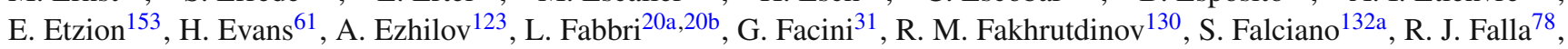
J. Faltova ${ }^{129}$, Y. Fang ${ }^{33 a}$, M. Fanti ${ }^{91 a}$,91b, A. Farbin ${ }^{8}$, A. Farilla ${ }^{134 a}$, T. Farooque ${ }^{12}$, S. Farrel1 ${ }^{15}$, S. M. Farrington ${ }^{170}$, P. Farthouat ${ }^{30}$, F. Fassi ${ }^{135 e}$, P. Fassnacht ${ }^{30}$, D. Fassouliotis ${ }^{9}$, M. Faucci Giannelli ${ }^{77}$, A. Favareto ${ }^{50 a, 50 b}$, L. Fayard ${ }^{117}$,

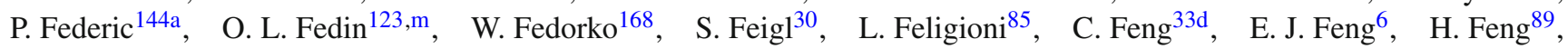
A. B. Fenyuk ${ }^{130}$, L. Feremenga ${ }^{8}$, P. Fernandez Martinez ${ }^{167}$, S. Fernandez Perez $^{30}$, J. Ferrando ${ }^{53}$, A. Ferrari ${ }^{166}$, P. Ferrari ${ }^{107}$, 
R. Ferrari ${ }^{121 a}$, D. E. Ferreira de Lima ${ }^{53}$, A. Ferrer ${ }^{167}$, D. Ferrere ${ }^{49}$, C. Ferretti ${ }^{89}$, A. Ferretto Parodi ${ }^{50 a, 50 b}$, M. Fiascaris ${ }^{31}$, F. Fiedler ${ }^{83}$, A. Filipčič $\check{c}^{75}$, M. Filipuzzi ${ }^{42}$, F. Filthaut ${ }^{106}$, M. Fincke-Keeler ${ }^{169}$, K. D. Finellii ${ }^{150}$, M. C. N. Fiolhais ${ }^{126 a, 126 c}$, L. Fiorini ${ }^{167}$, A. Firan ${ }^{40}$, A. Fischer ${ }^{2}$, C. Fischer $^{12}$, J. Fischer ${ }^{175}$, W. C. Fisher ${ }^{90}$, E. A. Fitzgerald ${ }^{23}$, I. Fleck ${ }^{141}$, P. Fleischmann ${ }^{89}$, S. Fleischmann ${ }^{175}$, G. T. Fletcher ${ }^{139}$, G. Fletcher ${ }^{76}$, R. R. M. Fletcher ${ }^{122}$, T. Flick ${ }^{175}$, A. Floderus ${ }^{81}$, L. R. Flores Castillo ${ }^{60 a}$ ， M. J. Flowerdew ${ }^{101}$ ， A. Formica ${ }^{136}$ ， A. Forti ${ }^{84}$ ， D. Fournier ${ }^{117} ，$ H. Fox ${ }^{72}$ ， S. Fracchia ${ }^{12}$, P. Francavilla ${ }^{80}$, M. Franchini ${ }^{20 a, 20 b}$, D. Francis ${ }^{30}$, L. Franconi ${ }^{119}$, M. Franklin ${ }^{57}$, M. Frate ${ }^{163}$, M. Fraternali ${ }^{121 a, 121 b}$, D. Freeborn ${ }^{78}$, S. T. French ${ }^{28}$, F. Friedrich ${ }^{44}$, D. Froidevaux ${ }^{30}$, J. A. Frost ${ }^{120}$, C. Fukunaga ${ }^{156}$, E. Fullana Torregrosa ${ }^{83}$, B. G. Fulsom ${ }^{143}$, J. Fuster ${ }^{167}$, C. Gabaldon ${ }^{55}$, O. Gabizon ${ }^{175}$, A. Gabrielli ${ }^{20 a, 20 b}$, A. Gabrielli ${ }^{132 a, 132 b}$, S. Gadatsch ${ }^{107}$, S. Gadomski ${ }^{49}$, G. Gagliardi ${ }^{50 a, 50 b}$, P. Gagnon ${ }^{61}$, C. Galea ${ }^{106}$, B. Galhardo $126 a, 126 c$, E. J. Gallas ${ }^{120}$, B. J. Gallop ${ }^{131}$, P. Gallus ${ }^{128}$, G. Galster ${ }^{36}$, K. K. Gan ${ }^{111}$, J. Gao ${ }^{33 b, 85}$, Y. Gao ${ }^{46}$, Y. S. Gao ${ }^{143, e}$, F. M. Garay Walls ${ }^{46}$, F. Garberson ${ }^{176}$, C. García ${ }^{167}$, J. E. García Navarro ${ }^{167}$, M. Garcia-Sciveres ${ }^{15}$, R. W. Gardner ${ }^{31}$, N. Garelli ${ }^{143}$, V. Garonne ${ }^{119}$, C. Gatti ${ }^{47}$, A. Gaudiello ${ }^{50 a, 50 b}$, G. Gaudio ${ }^{121 a}$, B. Gaur ${ }^{141}$, L. Gauthier ${ }^{95}$, P. Gauzzi132a,132b, I. L. Gavrilenko ${ }^{96}$, C. Gay ${ }^{168}$,

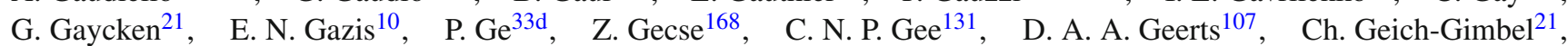
M. P. Geisler ${ }^{58 a}$, C. Gemme ${ }^{50 a}$, M. H. Genest ${ }^{55}$, S. Gentile ${ }^{132 a, 132 b}$, M. George ${ }^{54}$, S. George ${ }^{77}$, D. Gerbaudo ${ }^{163}$, A. Gershon ${ }^{153}$, H. Ghazlane ${ }^{135 b}$, B. Giacobbe ${ }^{20 a}$, S. Giagu ${ }^{132 a, 132 b}$, V. Giangiobbe ${ }^{12}$, P. Giannetti1 ${ }^{124 a, 124 b}$, B. Gibbard ${ }^{25}$, S. M. Gibson ${ }^{77}$, M. Gilchriese ${ }^{15}$, T. P. S. Gillam ${ }^{28}$, D. Gillberg ${ }^{30}, \quad$ G. Gilles ${ }^{34}$, D. M. Gingrich ${ }^{3, d}$, N. Giokaris ${ }^{9}$, M. P. Giordani ${ }^{164 a, 164 c}$, F. M. Giorgi ${ }^{20 a}$ ， F. M. Giorgi ${ }^{16}$, P. F. Giraud ${ }^{136}$, P. Giromini ${ }^{47}$, D. Giugni91a , C. Giuliani ${ }^{48}$, M. Giulini ${ }^{58 b}$, B. K. Gjelsten ${ }^{119}$, S. Gkaitatzis ${ }^{154}$, I. Gkialas ${ }^{154}$, E. L. Gkougkousis ${ }^{117}$, L. K. Gladilinn ${ }^{99}$, C. Glasman ${ }^{82}$, J. Glatzer ${ }^{30}$, P. C. F. Glaysher ${ }^{46}$, A. Glazov ${ }^{42}$, M. Goblirsch-Kolb ${ }^{101}$, J. R. Goddard ${ }^{76}$, J. Godlewski ${ }^{39}$, S. Goldfarb ${ }^{89}$, T. Golling ${ }^{49}$, D. Golubkov ${ }^{130}$, A. Gomes ${ }^{126 a, 126 b, 126 d,} \quad$ R. Gonçalo ${ }^{126 a}$, J. Goncalves Pinto Firmino Da Costa ${ }^{136}$, L. Gonella ${ }^{21}$, S. González de la Hoz ${ }^{167}$, G. Gonzalez Parra' ${ }^{12}$, S. Gonzalez-Sevilla ${ }^{49}$, L. Goossens ${ }^{30}$, P. A. Gorbounov ${ }^{97}$, H. A. Gordon ${ }^{25}$, I. Gorelov ${ }^{105}$, B. Gorini ${ }^{30}$, E. Gorini ${ }^{73 a}, 73 b$, A. Gorišek ${ }^{75}$, E. Gornicki ${ }^{39}$, A. T. Goshaw ${ }^{45}$, C. Gössling ${ }^{43}$, M. I. Gostkin ${ }^{65}$, D. Goujdami ${ }^{135 c}$, A. G. Goussiou ${ }^{138}$, N. Govender ${ }^{145 b}$, E. Gozani ${ }^{152}$, H. M. X. Grabas ${ }^{137}$, L. Graber ${ }^{54}$, I. Grabowska-Bold ${ }^{38 a}$, P. Grafström ${ }^{20 a, 20 b}$, K-J. Grahn ${ }^{42}$, J. Gramling ${ }^{49}$, E. Gramstad ${ }^{119}$, S. Grancagnolo ${ }^{16}$, V. Grassi ${ }^{148}$, V. Gratchev ${ }^{123}$, H. M. Gray ${ }^{30}$, E. Graziani ${ }^{134 a}$, Z. D. Greenwood ${ }^{79, n}$, K. Gregersen ${ }^{78}$, I. M. Gregor ${ }^{42}$, P. Grenier ${ }^{143}$, J. Griffiths ${ }^{8}$, A. A. Grillo ${ }^{137}$, K. Grimm ${ }^{72}$, S. Grinstein ${ }^{12, o}$, Ph. Gris ${ }^{34}$, J.-F. Grivaz ${ }^{117}$, J. P. Grohs ${ }^{44}$, A. Grohsjean ${ }^{42}$, E. Gross ${ }^{172}$, J. Grosse-Knetter ${ }^{54}$, G. C. Grossi ${ }^{79}$, Z. J. Grout ${ }^{149}$, L. Guan ${ }^{33 \mathrm{~b}}$, J. Guenther ${ }^{128}$, F. Guescini' ${ }^{49}$, D. Guest ${ }^{176}$, O. Gueta ${ }^{153}$, E. Guido ${ }^{50 a, 50 b}$, T. Guillemin ${ }^{117}, \quad$ S. Guindon ${ }^{2}, \quad$ U. Gul ${ }^{53}, \quad$ C. Gumpert ${ }^{44}$, J. Guo ${ }^{33 e}, \quad$ S. Gupta ${ }^{120}$, G. Gustavino ${ }^{132 a, 132 b}$, P. Gutierrez ${ }^{113}$ ， N. G. Gutierrez Ortiz ${ }^{53}, \quad$ C. Gutschow ${ }^{44}, \quad$ C. Guyot ${ }^{136}, \quad$ C. Gwenlan ${ }^{120}$, C. B. Gwilliam ${ }^{74}$, A. Haas ${ }^{110}$, C. Haber ${ }^{15}$, H. K. Hadavand ${ }^{8}$, N. Haddad ${ }^{135 e}$, P. Haefnerer ${ }^{21}$, S. Hageböck ${ }^{21}$, Z. Hajduk ${ }^{39}$, H. Hakobyan ${ }^{177}$, M. Haleem ${ }^{42}$, J. Haley ${ }^{114}$, D. Hall ${ }^{120}$, G. Halladjian ${ }^{90}$, G. D. Hallewell ${ }^{85}$, K. Hamacher ${ }^{175}$, P. Hamal ${ }^{115}$, K. Hamano ${ }^{169}$, M. Hamer ${ }^{54}$, A. Hamilton ${ }^{145 a}$, G. N. Hamity ${ }^{145 c}$, P. G. Hamnett ${ }^{42}$, L. Han ${ }^{33 b}$, K. Hanagaki ${ }^{118}$, K. Hanawa ${ }^{155}$, M. Hance ${ }^{15}$, P. Hanke ${ }^{58 a}$, R. Hanna ${ }^{136}$, J. B. Hansen ${ }^{36}$, J. D. Hansen ${ }^{36}$, M. C. Hansen ${ }^{21}$, P. H. Hansen ${ }^{36}$,

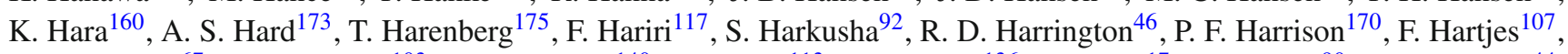
M. Hasegawa ${ }^{67}$, S. Hasegawa ${ }^{103}$, Y. Hasegawa ${ }^{140}$, A. Hasib ${ }^{113}$, S. Hassani ${ }^{136}$, S. Haug ${ }^{17}$, R. Hauser ${ }^{90}$, L. Hauswald ${ }^{44}$, M. Havranek ${ }^{127}$, C. M. Hawkes ${ }^{18}$, R. J. Hawkings ${ }^{30}$, A. D. Hawkins ${ }^{81}$, T. Hayashi ${ }^{160}$, D. Hayden ${ }^{90}$, C. P. Hays ${ }^{120}$, J. M. Hays ${ }^{76}$, H. S. Hayward ${ }^{74}$, S. J. Haywood ${ }^{131}$, S. J. Head ${ }^{18}$, T. Heck ${ }^{83}$, V. Hedberg ${ }^{81}$, L. Heelan ${ }^{8}$, S. Heim ${ }^{122}$, T. Heim ${ }^{175}$, B. Heinemann ${ }^{15}$, L. Heinrich ${ }^{110}$, J. Hejbal ${ }^{127}$, L. Helary ${ }^{22}$, S. Hellman ${ }^{146 a, 146 b}$, D. Hellmich ${ }^{21}$, C. Helsens ${ }^{30}$, J. Henderson ${ }^{120}$, R. C. W. Henderson ${ }^{72}$, Y. Heng ${ }^{173}$, C. Hengler ${ }^{42}$, A. Henrichs ${ }^{176}$, A. M. Henriques Correia ${ }^{30}$, S. Henrot-Versille ${ }^{117}$, G. H. Herbert ${ }^{16}$, Y. Hernández Jiménez ${ }^{167}$, R. Herrberg-Schubert ${ }^{16}$, G. Herten ${ }^{48}$, R. Hertenberger ${ }^{100}$, L. Hervas ${ }^{30}$, G. G. Hesketh ${ }^{78}$ ， N. P. Hessey ${ }^{107}$ ， J. W. Hetherly ${ }^{40}$ ， R. Hickling ${ }^{76}$, E. Higón-Rodriguez ${ }^{167}$, E. Hill ${ }^{169}$, J. C. Hill ${ }^{28}$, K. H. Hiller ${ }^{42}$, S. J. Hillier ${ }^{18}$, I. Hinchliffe ${ }^{15}$, E. Hines ${ }^{122}$, R. R. Hinman ${ }^{15}$, M. Hirose ${ }^{157}$, D. Hirschbuehl ${ }^{175}$, J. Hobbs ${ }^{148}$, N. Hod ${ }^{107}$, M. C. Hodgkinson ${ }^{139}$, P. Hodgson ${ }^{139}$, A. Hoecker ${ }^{30}$, M. R. Hoeferkamp ${ }^{105}$, F. Hoenig ${ }^{100}$, M. Hohlfeld ${ }^{83}$, D. Hohn ${ }^{21}$, T. R. Holmes ${ }^{15}$, M. Homann ${ }^{43}$, T. M. Hong ${ }^{125}$, L. Hooft van Huysduynen ${ }^{110}$, W. H. Hopkins ${ }^{116}$, Y. Horii ${ }^{103}$, A. J. Horton ${ }^{142}$, J-Y. Hostachy ${ }^{55}$, S. Hou ${ }^{151}$, A. Hoummada ${ }^{135 a}$, J. Howard ${ }^{120}$, J. Howarth ${ }^{42}$, M. Hrabovsky ${ }^{115}$, I. Hristova ${ }^{16}$, J. Hrivnac ${ }^{117}$, T. Hryn'ova ${ }^{5}$, A. Hrynevich ${ }^{93}$, C. Hsu ${ }^{145 c}$, P. J. Hsu ${ }^{151, p}$, S.-C. Hsu ${ }^{138}$, D. Hu ${ }^{35}$, Q. Hu ${ }^{33 b}$, X. Hu ${ }^{89}$, Y. Huang ${ }^{42}$, Z. Hubacek ${ }^{30}$, F. Hubaut ${ }^{85}$, F. Huegging ${ }^{21}$, T. B. Huffman ${ }^{120}$, E. W. Hughes ${ }^{35}$, G. Hughes ${ }^{72}$,

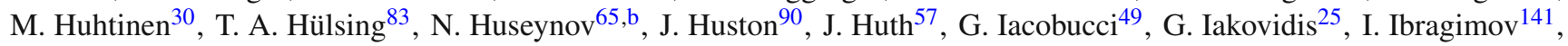
L. Iconomidou-Fayard ${ }^{117}$, E. Ideal ${ }^{176}$, Z. Idrissi ${ }^{135 e}$, P. Iengo ${ }^{30}$, O. Igonkina ${ }^{107}$, T. Iizawa ${ }^{171}$, Y. Ikegami ${ }^{66}$, K. Ikematsu $^{141}$, M. Ikeno ${ }^{66}$, Y. Ilchenko ${ }^{31, q}$, D. Iliadis ${ }^{154}$, N. Ilic ${ }^{143}$, Y. Inamaru ${ }^{67}$, T. Ince ${ }^{101}$, P. Ioannou ${ }^{9}$, M. Iodice ${ }^{134 a}$, K. Iordanidou ${ }^{35}$, V. Ippolito ${ }^{57}$, A. Irles Quiles ${ }^{167}$, C. Isaksson ${ }^{166}$, M. Ishino ${ }^{68}$, M. Ishitsuka ${ }^{157}$, R. Ishmukhametov ${ }^{111}$, C. Issever ${ }^{120}$, S. Istin ${ }^{19 a}$, J. M. Iturbe Ponce ${ }^{84}$, R. Iuppa ${ }^{133 a, 133 b}$, J. Ivarsson ${ }^{81}$, W. Iwanski ${ }^{39}$, H. Iwasaki ${ }^{66}$, J. M. Izen ${ }^{41}$, V. Izzo ${ }^{104 a}$, S. Jabbar ${ }^{3}$, B. Jackson ${ }^{122}$, M. Jackson ${ }^{74}$, P. Jackson ${ }^{1}$, M. R. Jaekel ${ }^{30}$, V. Jain ${ }^{2}$, K. Jakobs ${ }^{48}$, S. Jakobsen ${ }^{30}$, T. Jakoubek ${ }^{127}$, 
J. Jakubek ${ }^{128}$ ， D. O. Jamin ${ }^{151} ， \quad$ D. K. Jana ${ }^{79} ，$ E. Jansen ${ }^{78} ， \quad$ R. Jansky ${ }^{62} ， \quad$ J. Janssen ${ }^{21} ， \quad$ M. Janus ${ }^{170 ， \quad \text { G. Jarlskog }}{ }^{81}$ ， N. Javadov ${ }^{65, b}$, T. Javůrek ${ }^{48}$ ， L. Jeanty ${ }^{15}$ ， J. Jejelava ${ }^{51 a, r}$, G.-Y. Jeng ${ }^{150}$ ， D. Jennens ${ }^{88}$, P. Jenni ${ }^{48, s}$, J. Jentzsch ${ }^{43}$, C. Jeske ${ }^{170}$ ，S. Jézéquel ${ }^{5}$ ， H. Ji ${ }^{173}$ ， J. Jia ${ }^{148}$ ，Y. Jiang ${ }^{33 b} ，$ S. Jiggins ${ }^{78}$ ， J. Jimenez Pena ${ }^{167} ，$ S. Jin ${ }^{33 a} ，$ A. Jinaru ${ }^{26 a}$, O. Jinnouchi ${ }^{157}$, M. D. Joergensen ${ }^{36}$, P. Johansson ${ }^{139}$, K. A. Johns ${ }^{7}$, K. Jon-And ${ }^{146 a, 146 b}$, G. Jones ${ }^{170}$, R. W. L. Jones ${ }^{72}$, T. J. Jones ${ }^{74}$ ，J. Jongmanns ${ }^{58 a}$ ，P. M. Jorge ${ }^{126 a, 126 b} ，$ K. D. Joshi ${ }^{84}$ ，J. Jovicevic ${ }^{159 a} ， X . J^{173} ，$ C. A. Jung ${ }^{43}$ ，P. Jussel ${ }^{62}$, A. Juste Rozas ${ }^{12,0}$, M. Kaci ${ }^{167}$, A. Kaczmarska ${ }^{39}$, M. Kado ${ }^{117}$, H. Kagan ${ }^{111}$, M. Kagan ${ }^{143}$, S. J. Kahn ${ }^{85}$, E. Kajomovitz ${ }^{45}$, C. W. Kalderon ${ }^{120}$, S. Kama ${ }^{40}$, A. Kamenshchikov ${ }^{130}$, N. Kanaya ${ }^{155}$, M. Kaneda ${ }^{30}$, S. Kaneti ${ }^{28}$, V. A. Kantserov ${ }^{98}$, J. Kanzaki ${ }^{66}$, B. Kaplan ${ }^{110}$, A. Kapliy ${ }^{31}$, D. Kar ${ }^{53}$, K. Karakostas ${ }^{10}$, A. Karamaoun ${ }^{3}$, N. Karastathis ${ }^{10,107}$, M. J. Kareem ${ }^{54}$, M. Karnevskiy ${ }^{83}$, S. N. Karpov ${ }^{65}$, Z. M. Karpova ${ }^{65}$, K. Karthik ${ }^{110}$, V. Kartvelishvilii2 , A. N. Karyukhin ${ }^{130}$, L. Kashif ${ }^{173}$, R. D. Kass ${ }^{111}$, A. Kastanas ${ }^{14}$, Y. Kataoka ${ }^{155}$, A. Katre ${ }^{49}$, J. Katzy ${ }^{42}$, K. Kawagoe ${ }^{70}$, T. Kawamoto ${ }^{155}$, G. Kawamura ${ }^{54}$, S. Kazama ${ }^{155}$, V. F. Kazanin ${ }^{109, c}$, M. Y. Kazarinov ${ }^{65}$, R. Keeler ${ }^{169}$ ， R. Kehoe ${ }^{40}$, J. S. Keller ${ }^{42}$ ， J. J. Kempster ${ }^{77}$, H. Keoshkerian ${ }^{84}$, O. Kepka ${ }^{127}$, B. P. Kerševan ${ }^{75}$, S. Kersten ${ }^{175}$, R. A. Keyes ${ }^{87}$, F. Khalil-zada ${ }^{11}$, H. Khandanyan ${ }^{146 a, 146 b}$, A. Khanov ${ }^{114}$, A. G. Kharlamov ${ }^{109, c}$, T. J. Khoo ${ }^{28}$, V. Khovanskiy ${ }^{97}$, E. Khramov ${ }^{65}$, J. Khubua ${ }^{51 b, t}$, H. Y. Kim ${ }^{8}$, H. Kim ${ }^{146 a}{ }_{146 b}$, S. H. Kim ${ }^{160}$, Y. K. Kim ${ }^{31}$, N. Kimura ${ }^{154}$, O. M. Kind ${ }^{16}$, B. T. King ${ }^{74}$, M. King ${ }^{167}$, S. B. King ${ }^{168}$, J. Kirk ${ }^{131}$, A. E. Kiryunin ${ }^{101}$, T. Kishimoto ${ }^{67}$, D. Kisielewska ${ }^{38 a}$, F. Kiss ${ }^{48}$, K. Kiuchi ${ }^{160}$, O. Kivernyk ${ }^{136}$, E. Kladiva ${ }^{144 b}$, M. H. Klein ${ }^{35}$, M. Klein ${ }^{74}$, U. Klein ${ }^{74}$, K. Kleinknecht ${ }^{83}$ ， P. Klimek ${ }^{146 a, 146 b}$, A. Klimentov ${ }^{25}$, R. Klingenberg ${ }^{43}$, J. A. Klinger ${ }^{139}$, T. Klioutchnikova ${ }^{30}$, E.-E. Kluge ${ }^{58 a}$, P. Kluit ${ }^{107}$, S. Kluth ${ }^{101}$, E. Kneringer ${ }^{62}$, E. B. F. G. Knoops ${ }^{85}$, A. Knue ${ }^{53}$, A. Kobayashi ${ }^{155}$, D. Kobayashi ${ }^{157}$, T. Kobayashi ${ }^{155}$, M. Kobel ${ }^{44}$, M. Kocian ${ }^{143}$, P. Kodys ${ }^{129}$, T. Koffas ${ }^{29}$, E. Koffeman ${ }^{107}$, L. A. Kogan ${ }^{120}$, S. Kohlmann ${ }^{175}$, Z. Kohout ${ }^{128}$, T. Kohriki ${ }^{66}$, T. Koi ${ }^{143}$, H. Kolanoski ${ }^{16}$, I. Koletsou ${ }^{5}$, A. A. Komar ${ }^{96, *}$, Y. Komori ${ }^{155}$, T. Kondo ${ }^{66}$, N. Kondrashova ${ }^{42}$, K. Köneke ${ }^{48}$, A. C. König ${ }^{106}$, S. König ${ }^{83}$, T. Kono ${ }^{66, u}$, R. Konoplich ${ }^{110, v}$, N. Konstantinidis ${ }^{78}$, R. Kopeliansky ${ }^{152}$, S. Koperny ${ }^{38 a}$, L. Köpke ${ }^{83}$, A. K. Kopp ${ }^{48}$, K. Korcyl ${ }^{39}$, K. Kordas ${ }^{154}$, A. Korn ${ }^{78}$, A. A. Korol ${ }^{109, c}$, I. Korolkov ${ }^{12}$, E. V. Korolkova ${ }^{139}$, O. Kortner ${ }^{101}$, S. Kortner ${ }^{101}$, T. Kosek ${ }^{129}$, V. V. Kostyukhin ${ }^{21}$, V. M. Kotov ${ }^{65}$, A. Kotwal ${ }^{45}$, A. Kourkoumeli-Charalampidi ${ }^{154}$, C. Kourkoumelis ${ }^{9}$, V. Kouskoura ${ }^{25}$, A. Koutsman ${ }^{159 a}$, R. Kowalewski ${ }^{169}$, T. Z. Kowalski ${ }^{38 a}$, W. Kozanecki ${ }^{136}, \quad$ A. S. Kozhin ${ }^{130}$, V. A. Kramarenko ${ }^{99}$, G. Kramberger $^{75}$, D. Krasnopevtsev ${ }^{98}$, M. W. Krasny ${ }^{80}$, A. Krasznahorkay ${ }^{30}$, J. K. Kraus ${ }^{21}$, A. Kravchenko ${ }^{25}$, S. Kreiss ${ }^{110}$, M. Kretz ${ }^{58 c}$, J. Kretzschmar ${ }^{74}$, K. Kreutzfeldt ${ }^{52}$, P. Krieger ${ }^{158}$, K. Krizka ${ }^{31}$, K. Kroeninger ${ }^{43}$, H. Kroha ${ }^{101}$, $\begin{array}{llll}\text { J. Kroll } & 122\end{array}$ J. Kroseberg ${ }^{21}$, J. Krstic ${ }^{13}$, U. Kruchonak ${ }^{65}, \quad$ H. Krüger ${ }^{21}$, N. Krumnack ${ }^{64}$, Z. V. Krumshteyn ${ }^{65}$, A. Kruse ${ }^{173}$, M. C. Kruse ${ }^{45}$, M. Kruskal ${ }^{22}$, T. Kubota ${ }^{88}, \quad$ H. Kucuk ${ }^{78}, \quad$ S. Kuday ${ }^{4 b}, \quad$ S. Kuehn ${ }^{48}, \quad$ A. Kugel ${ }^{58 c}$, F. Kuger ${ }^{174}$, A. Kuhl ${ }^{137}$, T. Kuhl ${ }^{42}$, V. Kukhtin ${ }^{65}$, Y. Kulchitsky ${ }^{92}$, S. Kuleshov ${ }^{32 b}$, M. Kuna ${ }^{132 a, 132 b}$, T. Kunigo ${ }^{68}$, A. Kupco ${ }^{127}$, H. Kurashige ${ }^{67}$, Y. A. Kurochkin ${ }^{92}$, R. Kurumida ${ }^{67}$, V. Kus ${ }^{127}$, E. S. Kuwertz ${ }^{169}$, M. Kuze ${ }^{157}$, J. Kvita ${ }^{115}$, T. Kwan ${ }^{169}$, D. Kyriazopoulos ${ }^{139}$, A. La Rosa ${ }^{49}$, J. L. La Rosa Navarro $24 d$, L. La Rotonda ${ }^{37 a, 37 b, ~ C . ~ L a c a s t a ~}{ }^{167}$, F. Lacava ${ }^{132 a, 132 b}$, J. Lacey ${ }^{29}$, H. Lacker ${ }^{16}$, D. Lacour ${ }^{80}$, V. R. Lacuesta ${ }^{167}$, E. Ladygin ${ }^{65}$, R. Lafaye ${ }^{5}$, B. Laforge ${ }^{80}$, T. Lagouri ${ }^{176}$, S. Lai ${ }^{48}$, L. Lambourne ${ }^{78}$, S. Lammers ${ }^{61}$, C. L. Lampen ${ }^{7}$, W. Lampl ${ }^{7}$, E. Lançon ${ }^{136}$, U. Landgraf ${ }^{48}$, M. P. J. Landon ${ }^{76}$, V. S. Lang ${ }^{58 a}$, J. C. Lange ${ }^{12}$, A. J. Lankford ${ }^{163}$, F. Lanni ${ }^{25}$, K. Lantzsch ${ }^{30}$, S. Laplace ${ }^{80}$, C. Lapoire ${ }^{30}$,

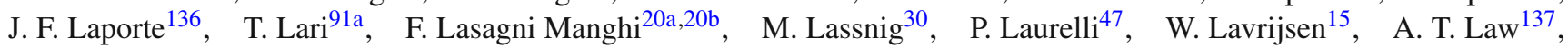
P. Laycock $^{74}$, T. Lazovich ${ }^{57}$, O. Le Dortz ${ }^{80}$, E. Le Guirriec ${ }^{85}$, E. Le Menedeu ${ }^{12}$, M. LeBlanc ${ }^{169}$, T. LeCompte ${ }^{6}$, F. Ledroit-Guillon ${ }^{55}$, C. A. Lee ${ }^{145 b}$, S. C. Lee ${ }^{151}$, L. Lee ${ }^{1}$, G. Lefebvre ${ }^{80}$, M. Lefebvre ${ }^{169}$, F. Legger ${ }^{100}$, C. Leggett ${ }^{15}$, A. Lehan ${ }^{74}$, G. Lehmann Miotto ${ }^{30}, \quad$ X. Lei ${ }^{7}$, W. A. Leight ${ }^{29}$, A. Leisos ${ }^{154, w}$, A. G. Leister ${ }^{176}, \quad$ M. A. L. Leite ${ }^{24 d}$, R. Leitner ${ }^{129}$, D. Lellouch ${ }^{172}$, B. Lemmer ${ }^{54}$, K. J. C. Leney ${ }^{78}$, T. Lenz ${ }^{21}$, B. Lenzi ${ }^{30}$, R. Leone ${ }^{7}$, S. Leone ${ }^{124 a, 124 b}$,

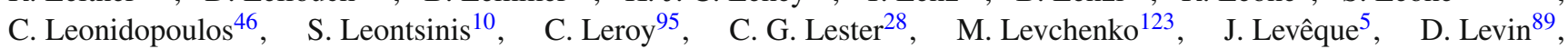

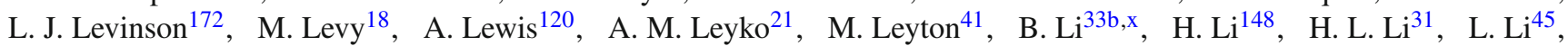
L. Li ${ }^{33 e}$, S. Li ${ }^{45}$, Y. Li ${ }^{33 c, y}$, Z. Liang ${ }^{137}$, H. Liao ${ }^{34}$, B. Liberti ${ }^{133 a}$, A. Liblong ${ }^{158}$, P. Lichard ${ }^{30}$, K. Lie ${ }^{165}$, J. Liebal ${ }^{21}$,

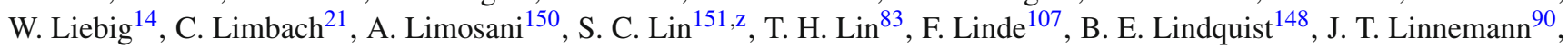
E. Lipeles ${ }^{122}$, A. Lipniacka ${ }^{14}$, M. Lisovyi ${ }^{58 b}$, T. M. Liss ${ }^{165}$, D. Lissauer ${ }^{25}$, A. Lister ${ }^{168}$, A. M. Litke ${ }^{137}$, B. Liu ${ }^{151, a a}$,

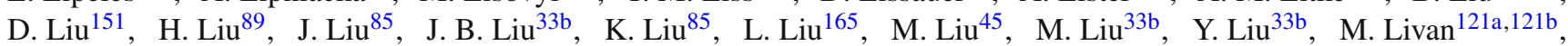
A. Lleres ${ }^{55}$, J. Llorente Merino ${ }^{82}$, S. L. Lloyd ${ }^{76}$, F. Lo Sterzo ${ }^{151}$, E. Lobodzinska ${ }^{42}$, P. Loch ${ }^{7}$, W. S. Lockman ${ }^{137}$, F. K. Loebinger ${ }^{84}$, A. E. Loevschall-Jensen ${ }^{36}$, A. Loginov ${ }^{176}$, T. Lohse ${ }^{16}$, K. Lohwasser ${ }^{42}$, M. Lokajicek ${ }^{127}$, B. A. Long ${ }^{22}$, J. D. Long ${ }^{89}$, R. E. Long ${ }^{72}$, K. A. Looper ${ }^{111}$, L. Lopes ${ }^{126 a}$, D. Lopez Mateos ${ }^{57}$, B. Lopez Paredes ${ }^{139}$, I. Lopez Paz ${ }^{12}$, J. Lorenz ${ }^{100}$, N. Lorenzo Martinez ${ }^{61}$, M. Losada ${ }^{162}$, P. Loscutoff ${ }^{15}$, P. J. Lösel ${ }^{100}$, X. Lou ${ }^{33 a}$, A. Lounis ${ }^{117}$, J. Love ${ }^{6}$, P. A. Love ${ }^{72}$, N. Lu ${ }^{89}$, H. J. Lubatti ${ }^{138}$, C. Luci ${ }^{132 a, 132 b}$, A. Lucotte ${ }^{55}$, F. Luehring ${ }^{61}$, W. Lukas ${ }^{62}$, L. Luminari ${ }^{132 a}$, O. Lundberg ${ }^{146 a, 146 b}$, B. Lund-Jensen ${ }^{147}$, D. Lynn ${ }^{25}$, R. Lysak ${ }^{127}$, E. Lytken ${ }^{81}$, H. Ma ${ }^{25}$, L. L. Ma ${ }^{33 d}$, G. Maccarrone ${ }^{47}$, A. Macchiolo ${ }^{101}$, C. M. Macdonald ${ }^{139}$, J. Machado Miguens ${ }^{122,126 b}, \quad$ D. Macina ${ }^{30}$, D. Madaffari ${ }^{85}$, R. Madar ${ }^{34}$, H. J. Maddocks ${ }^{72}$, W. F. Mader ${ }^{44}$, A. Madsen ${ }^{166}$, S. Maeland ${ }^{14}$, T. Maeno ${ }^{25}$, A. Maevskiy ${ }^{99}$, E. Magradze ${ }^{54}$, K. Mahboubi ${ }^{48}$, 
J. Mahlstedt ${ }^{107}$ ，C. Maiani ${ }^{136}$ ，C. Maidantchik ${ }^{24 a}$ ，A. A. Maier ${ }^{101}$ ，T. Maier ${ }^{100}$ ，A. Maio ${ }^{126 a, 126 b, 126 d}$ ，S. Majewski ${ }^{116}$, Y. Makida ${ }^{66}$, N. Makovec ${ }^{117}$, B. Malaescu ${ }^{80}$, Pa. Malecki ${ }^{39}$, V.P. Maleev ${ }^{123}$, F. Malek ${ }^{55}$, U. Mallik ${ }^{63}$, D. Malon ${ }^{6}$, C. Malone ${ }^{143}$, S. Maltezos ${ }^{10}$, V. M. Malyshev ${ }^{109}$, S. Malyukov ${ }^{30}$, J. Mamuzic ${ }^{42}$, G. Mancini ${ }^{47}$, B. Mandelli ${ }^{30}$, L. Mandelli19a, I. Mandić ${ }^{75}$, R. Mandrysch ${ }^{63}$, J. Maneira ${ }^{126 a, 126 b}$, A. Manfredini ${ }^{101}$, L. Manhaes de Andrade Filho ${ }^{24 b}$, J. Manjarres Ramos ${ }^{159 b}$, A. Mann ${ }^{100}$, P. M. Manning ${ }^{137}$, A. Manousakis-Katsikakis ${ }^{9}$, B. Mansoulie ${ }^{136}$, R. Mantifel ${ }^{87}$, M. Mantoani ${ }^{54}$, L. Mapelli ${ }^{30}$, L. March ${ }^{145 c}$, G. Marchiori ${ }^{80}$, M. Marcisovsky ${ }^{127}$, C. P. Marino ${ }^{169}$, M. Marjanovic ${ }^{13}$, D. E. Marley ${ }^{89}$, F. Marroquim ${ }^{24 a}$, S.P. Marsden ${ }^{84}$, Z. Marshall ${ }^{15}$, L. F. Marti ${ }^{17}$, S. Marti-Garcia ${ }^{167}$, B. Martin ${ }^{90}$,

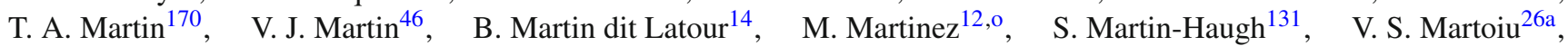
A. C. Martyniuk ${ }^{78}$, M. Marx ${ }^{138}$, F. Marzano ${ }^{132 a}$, A. Marzin $^{30}$, L. Masetti ${ }^{83}$, T. Mashimo ${ }^{155}$, R. Mashinistov ${ }^{96}$, J. Masik ${ }^{84}$, A. L. Maslennikov ${ }^{109, c}$, I. Massa ${ }^{20 a, 20 b}$ ，L. Massa ${ }^{20 a, 20 b}$ ，N. Massol ${ }^{5}$ ，P. Mastrandrea ${ }^{148}$ ，A. Mastroberardino ${ }^{37 a, 37 b}$, T. Masubuc hi ${ }^{155}$, P. Mättig ${ }^{175}$, J. Mattmann ${ }^{83}$ ， J. Maurer ${ }^{26 a}$, S. J. Maxfield ${ }^{74}$, D. A. Maximov ${ }^{109, c}$, R. Mazini ${ }^{151}$, S. M. Mazza ${ }^{11 a, 91 b}$, L. Mazzaferro ${ }^{133 a, 133 b}$, G. Mc Goldrick ${ }^{158}$, S. P. Mc Kee ${ }^{89}$, A. McCarn ${ }^{89}$, R. L. McCarthy ${ }^{148}$, T. G. McCarthy ${ }^{29}$, N. A. McCubbin ${ }^{131}$, K. W. McFarlane ${ }^{56, *}$, J. A. Mcfayden ${ }^{78}$, G. Mchedlidze ${ }^{54}$, S. J. McMahon ${ }^{131}$, R. A. McPherson ${ }^{169, \mathrm{k}}$, M. Medinnis ${ }^{42}$, S. Meehan ${ }^{145 a}$, S. Mehlhase ${ }^{100}$, A. Mehta ${ }^{74}$, K. Meier ${ }^{58 a}$, C. Meineck ${ }^{100}$, B. Meirose ${ }^{41}$, B. R. Mellado Garcia ${ }^{145 c}$, F. Meloni ${ }^{17}$, A. Mengarelli ${ }^{20 a, 20 b}$, S. Menke ${ }^{101}$, E. Meoni ${ }^{161}$, K. M. Mercurio ${ }^{57}$, S. Mergelmeyer ${ }^{21}$, P. Mermod ${ }^{49}$, L. Merola ${ }^{104 a, 104 b}$, C. Meroni ${ }^{91 a}$, F. S. Merritt ${ }^{31}$, A. Messina ${ }^{132 a, 132 b}$, J. Metcalfe ${ }^{25}$, A. S. Mete ${ }^{163}$, C. Meyer ${ }^{83}$, C. Meyer ${ }^{122}$, J-P. Meyer ${ }^{136}$, J. Meyer ${ }^{107}$, R. P. Middleton ${ }^{131}$, S. Miglioranzi ${ }^{164 a, 164 c}$, L. Mijović ${ }^{21}$, G. Mikenberg ${ }^{172}$, M. Mikestikova ${ }^{127}$, M. Mikuž ${ }^{75}$, M. Milesi ${ }^{88}$, A. Milic ${ }^{30}$, D. W. Miller ${ }^{31}$, C. Mills ${ }^{46}$,

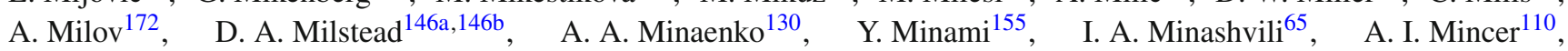
B. Mindur ${ }^{38 a}$, M. Mineev65, Y. Ming ${ }^{173}$, L. M. Mir ${ }^{12}$, T. Mitani ${ }^{171}$, J. Mitrevski ${ }^{100}$, V. A. Mitsou ${ }^{167}$, A. Miucci ${ }^{49}$, P. S. Miyagawa ${ }^{139}$, J. U. Mjörnmark ${ }^{81}$, T. Moa ${ }^{146 a, 146 b}$, K. Mochizuki $^{85}$, S. Mohapatra ${ }^{35}$, W. Mohr ${ }^{48}$, S. Molander ${ }^{146 a, 146 b}$, R. Moles-Valls ${ }^{167}$, K. Mönig42, C. Monini ${ }^{45}$, J. Monk ${ }^{36}$, E. Monnier ${ }^{85}$, J. Montejo Berlingen ${ }^{12}$, F. Monticelli ${ }^{71}$, S. Monzani ${ }^{132 a, 132 b}$, R. W. Moore ${ }^{3}$, N. Morange ${ }^{117}$, D. Moreno ${ }^{162}$, M. Moreno Llácer ${ }^{54}$, P. Morettini ${ }^{50 a}$, M. Morgenstern ${ }^{44}$, M. Morii ${ }^{57}$, M. Morinaga ${ }^{155}$, V. Morisbak ${ }^{119}$, S. Moritz ${ }^{83}$, A. K. Morley ${ }^{147}$, G. Mornacchi ${ }^{30}$, J. D. Morris ${ }^{76}$, S. S. Mortensen ${ }^{36}$, A. Morton ${ }^{53}$, L. Morvaj ${ }^{103}$, M. Mosidze ${ }^{51 b}$, J. Moss ${ }^{111}$, K. Motohashi ${ }^{157}$, R. Mount ${ }^{143}$, E. Mountricha ${ }^{25}$, S. V. Mouraviev ${ }^{96, *}$, E. J. W. Moyse ${ }^{86}$, S. Muanza ${ }^{85}$, R. D. Mudd ${ }^{18}$, F. Mueller ${ }^{101}$, J. Mueller ${ }^{125}$, K. Mueller ${ }^{21}$, R. S. P. Mueller ${ }^{100}$, T. Mueller ${ }^{28}$, D. Muenstermann ${ }^{49}$, P. Mullen ${ }^{53}$, G. A. Mullier ${ }^{17}$, Y. Munwes ${ }^{153}$, J. A. Murillo Quijada ${ }^{18}$, W. J. Murray ${ }^{170,131}$, H. Musheghyan ${ }^{54}$, E. Musto ${ }^{152}$, A. G. Myagkov ${ }^{130, a b}$, M. Myska ${ }^{128}$, O. Nackenhorst ${ }^{54}$, J. Nadal ${ }^{54}$, K. Nagai ${ }^{120}$, R. Nagai ${ }^{157}$, Y. Nagai ${ }^{85}$, K. Nagano ${ }^{66}$, A. Nagarkar ${ }^{111}$, Y. Nagasaka ${ }^{59}$, K. Nagata ${ }^{160}$, M. Nagel ${ }^{101}$, E. Nagy ${ }^{85}$, A. M. Nairz ${ }^{30}$, Y. Nakahama ${ }^{30}$, K. Nakamura ${ }^{66}$, T. Nakamura ${ }^{155}$, I. Nakano ${ }^{112}$, H. Namasivayam ${ }^{41}$, R. F. Naranjo Garcia ${ }^{42}$, R. Narayan ${ }^{31}$, T. Naumann ${ }^{42}$, G. Navarro ${ }^{162}$, R. Nayyar ${ }^{7}$, H. A. Neal ${ }^{89}$, P. Yu. Nechaeva ${ }^{96}$, T. J. Neep ${ }^{84}$, P. D. Nef ${ }^{143}$, A. Negri ${ }^{121 a, 121 b}$, M. Negrini ${ }^{20 a}$, S. Nektarijevic ${ }^{106}, \quad$ C. Nellist ${ }^{117}$, A. Nelson ${ }^{163}$, S. Nemecek ${ }^{127}$, P. Nemethy ${ }^{110}$, A. A. Nepomuceno ${ }^{24 a}$, M. Nessi ${ }^{30, a c}$, M. S. Neubauer ${ }^{165}$, M. Neumann ${ }^{175}$, R. M. Neves ${ }^{110}$, P. Nevski ${ }^{25}$, P. R. Newman ${ }^{18}$, D. H. Nguyen ${ }^{6}$, R. B. Nickerson ${ }^{120}$, R. Nicolaidou ${ }^{136}$, B. Nicquevert ${ }^{30}$, J. Nielsen ${ }^{137}$, N. Nikiforou ${ }^{35}$, A. Nikiforov ${ }^{16}$, V. Nikolaenko ${ }^{130, a b}$, I. Nikolic-Audit ${ }^{80}$, K. Nikolopoulos ${ }^{18}$, J. K. Nilsen ${ }^{119}$, P. Nilsson ${ }^{25}$, Y. Ninomiya ${ }^{155}$, A. Nisati ${ }^{132 a}$, R. Nisius ${ }^{101}$, T. Nobe ${ }^{157}$, M. Nomachi ${ }^{118}$, I. Nomidis ${ }^{29}$, T. Nooney ${ }^{76}$, S. Norberg ${ }^{113}$, M. Nordberg ${ }^{30}$, O. Novgorodova ${ }^{44}$, S. Nowak ${ }^{101}$, M. Nozaki ${ }^{66}$, L. Nozka ${ }^{115}$, K. Ntekas ${ }^{10}$, G. Nunes Hanninger ${ }^{88}$, T. Nunnemann ${ }^{100}$, E. Nurse ${ }^{78}$, F. Nuti ${ }^{88}$, B. J. O'Brien ${ }^{46}$, F. O'grady ${ }^{7}$, D. C. O'Neil ${ }^{142}$, V. O'Shea ${ }^{53}$, F. G. Oakham ${ }^{29, \mathrm{~d}}$, H. Oberlack ${ }^{101}$, T. Obermann ${ }^{21}$, J. Ocariz ${ }^{80}$, A. Ochi ${ }^{67}$, I. Ochoa ${ }^{78}$, J. P. Ochoa-Ricoux ${ }^{32 a}$, S. Oda ${ }^{70}$, S. Odaka ${ }^{66}$, H. Ogren ${ }^{61}$, A. $\mathrm{Oh}^{84}$, S. H. Oh ${ }^{45}$, C. C. Ohm ${ }^{15}$, H. Ohman ${ }^{166}$, H. Oide ${ }^{30}$, W. Okamura ${ }^{18}$, H. Okawa ${ }^{160}$, Y. Okumura $^{31}$, T. Okuyama ${ }^{155}$, A. Olariu ${ }^{26 a}$, S. A. Olivares Pino ${ }^{46}$, D. Oliveira Damazio ${ }^{25}$, E. Oliver Garcia ${ }^{167}$, A. Olszewski ${ }^{39}$, J. Olszowska ${ }^{39}$, A. Onofre ${ }^{126 a, 126 e}$, P. U. E. Onyisi ${ }^{31, q}$, C. J. Oram ${ }^{159 a}$, M. J. Oreglia ${ }^{31}$, Y. Oren ${ }^{153}$, D. Orestano ${ }^{134 a, 134 b}$, N. Orlando ${ }^{154}$, C. Oropeza Barrera ${ }^{53}$, R. S. Orr ${ }^{158}$, B. Osculati ${ }^{50 a, 50 b}$, R. Ospanov ${ }^{84}$, G. Otero y Garzon $^{27}$, H. Otono $^{70}$, M. Ouchrif ${ }^{135 d}$, E. A. Ouellette ${ }^{169}$, F. Ould-Saada ${ }^{119}$, A. Ouraou ${ }^{136}$, K. P. Oussoren ${ }^{107}$, Q. Ouyang ${ }^{33 a}$, A. Ovcharova ${ }^{15}$, M. Owen ${ }^{53}$, R. E. Owen ${ }^{18}$, V. E. Ozcan ${ }^{19 a}$, N. Ozturk ${ }^{8}$, K. Pachal ${ }^{142}$, A. Pacheco Pages ${ }^{12}$, C. Padilla Aranda ${ }^{12}$, M. Pagáčová ${ }^{48}$, S. Pagan Griso ${ }^{15}$, E. Paganis ${ }^{139}$, C. Pahl ${ }^{101}$, F. Paige ${ }^{25}$, P. Pais ${ }^{86}$, K. Pajchel ${ }^{119}$, G. Palacino ${ }^{159 b}$, S. Palestini ${ }^{30}$, M. Palka ${ }^{38 b}$, D. Pallin ${ }^{34}$, A. Palma ${ }^{126 a, 126 b}$, Y. B. Pan ${ }^{173}$, E. Panagiotopoulou ${ }^{10}$, C. E. Pandini ${ }^{80}$, J. G. Panduro Vazquez ${ }^{77}$, P. Pani ${ }^{146 a}, 146 b$, S. Panitkin ${ }^{25}$, D. Pantea ${ }^{26 a}$, L. Paolozzi ${ }^{49}$, Th. D. Papadopoulou ${ }^{10}$, K. Papageorgiou ${ }^{154}$, A. Paramonov ${ }^{6}$, D. Paredes Hernandez ${ }^{154}$, M. A. Parker ${ }^{28}$, K. A. Parker ${ }^{139}$, F. Parodi ${ }^{50,50 b}$, J. A. Parsons ${ }^{35}$, U. Parzefall ${ }^{48}$, E. Pasqualucci ${ }^{132 a}$, S. Passaggio ${ }^{50 a}$, F. Pastore ${ }^{134 a, 134 b, *}$, Fr. Pastore ${ }^{77}$, G. Pásztor ${ }^{29}$ ， S. Pataraia ${ }^{175}$ ， N. D. Patel ${ }^{150}$ ， J. R. Pater ${ }^{84}$ ， T. Pauly ${ }^{30}$ ， J. Pearce ${ }^{169}$ ， B. Pearson ${ }^{113}$ ， L. E. Pedersen ${ }^{36}$, M. Pedersen ${ }^{119}$, S. Pedraza Lopez ${ }^{167}$, R. Pedro ${ }^{126 a, 126 b}$, S. V. Peleganchuk ${ }^{109, c}$, D. Pelikan ${ }^{166}$, H. Peng ${ }^{33 b}$, B. Penning ${ }^{31}$, J. Penwell ${ }^{61}$ ， D. V. Perepelitsa ${ }^{25}$ ， E. Perez Codina ${ }^{159 a}$ ， M. T. Pérez García-Estañ ${ }^{167}$ ， L. Perini ${ }^{91 a}$,91b， H. Pernegger ${ }^{30}$, S. Perrella ${ }^{104 a, 104 b}$, R. Peschke ${ }^{42}$, V. D. Peshekhonov ${ }^{65}$, K. Peters ${ }^{30}$, R. F. Y. Peters ${ }^{84}$, B. A. Petersen ${ }^{30}$, T. C. Petersen ${ }^{36}$, 
E. Petit ${ }^{42}$ ，A. Petridis ${ }^{146 a, 146 b}$ ， C. Petridou ${ }^{154}$ ， E. Petrolo ${ }^{132 a}$ ， F. Petrucci ${ }^{134 a, 134 b}, \quad$ N. E. Pettersson ${ }^{157}$ ， R. Pezoa ${ }^{32 b}$, P. W. Phillips ${ }^{131}$, G. Piacquadio ${ }^{143}$, E. Pianori ${ }^{170}$, A. Picazio ${ }^{49}$, E. Piccaro ${ }^{76}$, M. Piccinini ${ }^{20 a, 20 b}$, M. A. Pickering ${ }^{120}$, R. Piegaia ${ }^{27}$, D. T. Pignotti ${ }^{111}$, J. E. Pilcher ${ }^{31}$, A. D. Pilkington ${ }^{84}$, J. Pina ${ }^{126 a, 126 b, 126 d, ~ M . ~ P i n a m o n t i ~}{ }^{164 a, 164 c, a d}$, J. L. Pinfold ${ }^{3}$, A. Pingel ${ }^{36}$, B. Pinto ${ }^{126 a}$, S. Pires ${ }^{80}$, M. Pitt ${ }^{172}$, C. Pizio ${ }^{91 a}$,91b, L. Plazak ${ }^{144 a}$, M.-A. Pleier ${ }^{25}$, V. Pleskot ${ }^{129}$, E. Plotnikova $^{65}$, P. Plucinski 146a,146b, D. Pluth ${ }^{64}$, R. Poettgen ${ }^{146 a, 146 b}$, L. Poggioli ${ }^{117}$, D. Pohl ${ }^{21}$, G. Polesello ${ }^{121 a}$, A. Poley ${ }^{42}$, A. Policicchio ${ }^{37 a, 37 b}$, R. Polifka ${ }^{158}$, A. Polini ${ }^{20 a}$, C. S. Pollard ${ }^{53}$, V. Polychronakos ${ }^{25}$, K. Pommès ${ }^{30}$, L. Pontecorvo ${ }^{132 a}$, B. G. Pope ${ }^{90}$, G. A. Popeneciu ${ }^{26 b}$, D. S. Popovic ${ }^{13}$, A. Poppleton ${ }^{30}$, S. Pospisil ${ }^{128}$, K. Potamianos ${ }^{15}$, I. N. Potrap ${ }^{65}$, C. J. Potter ${ }^{149}$, C. T. Potter ${ }^{116}$, G. Poulard ${ }^{30}$, J. Poveda ${ }^{30}$, V. Pozdnyakov ${ }^{65}$, P. Pralavorio ${ }^{85}$, A. Pranko ${ }^{15}$,

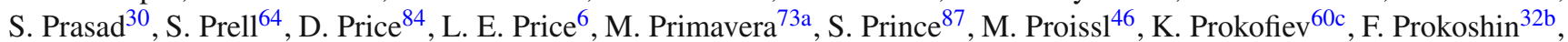
E. Protopapadaki ${ }^{136}$, S. Protopopescu ${ }^{25}$, J. Proudfoot ${ }^{6}$, M. Przybycien ${ }^{38 a}$, E. Ptacek ${ }^{116}$, D. Puddu ${ }^{134 a, 134 b}$, E. Pueschel ${ }^{86}$,

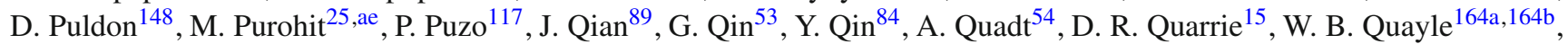
M. Queitsch-Maitland ${ }^{84}$, D. Quilty ${ }^{53}$, S. Raddum ${ }^{119}$, V. Radeka ${ }^{25}$, V. Radescu ${ }^{42}$, S. K. Radhakrishnan ${ }^{148}$, P. Radloff ${ }^{116}$, P. $\operatorname{Rados}^{88}$, F. Ragusa ${ }^{91 a, 91 b}$ ，G. Rahal ${ }^{178}$ ，S. Rajagopalan ${ }^{25}$ ， M. Rammensee ${ }^{30}$, C. Rangel-Smith ${ }^{166}$, F. Rauscher ${ }^{100}$, S. Rave ${ }^{83}$, T. Ravenscroft ${ }^{53}$, M. Raymond ${ }^{30}$, A. L. $\operatorname{Read}^{119}$, N. P. Readioff ${ }^{74}$, D. M. Rebuzzi1 121a,121b , A. Redelbach ${ }^{174}$, G. Redlinger ${ }^{25}$ ， R. Reece ${ }^{137}$, K. Reeves ${ }^{41}$ ， L. Rehnisch ${ }^{16}$ ， H. Reisin ${ }^{27}$, M. Relich ${ }^{163}$ ， C. Rembser ${ }^{30}$, H. Ren ${ }^{33 a}$, A. Renaud ${ }^{117}$ ， M. Rescigno ${ }^{132 a}$ ， S. Resconi ${ }^{91 a}$ ， O. L. Rezanova109,c ， P. Reznicek ${ }^{129}$ ， R. Rezvani ${ }^{95}$, R. Richter ${ }^{101}$, S. Richter ${ }^{78}$, E. Richter-Was ${ }^{38 b}$, O. Ricken ${ }^{21}$, M. Ridel ${ }^{80}$, P. Rieck ${ }^{16}$ ，C. J. Riegel ${ }^{175}$ ，J. Rieger ${ }^{54}$, M. Rijssenbeek ${ }^{148}$,

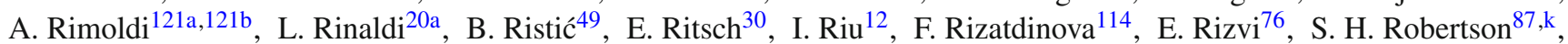
A. Robichaud-Veronneau ${ }^{87}$, D. Robinson ${ }^{28}$, J. E. M. Robinson ${ }^{84}$, A. Robson ${ }^{53}$, C. Roda ${ }^{124 a, 124 b}$, S. Roe ${ }^{30}$, O. Røhne ${ }^{119}$, S. Rolli ${ }^{161}$, A. Romaniouk ${ }^{98}$, M. Romano ${ }^{20 \mathrm{a}, 20 \mathrm{~b}}$, S. M. Romano Saez ${ }^{34}$, E. Romero Adam ${ }^{167, \quad \text { N. Rompotis }}{ }^{138}$, M. Ronzani ${ }^{48}$, L. $\operatorname{Roos}^{80}$, E. Ros ${ }^{167}$ ，S. Rosati ${ }^{132 a}$, K. Rosbach ${ }^{48}$, P. Rose ${ }^{137}$, P. L. Rosendahl ${ }^{14}$, O. Rosenthal ${ }^{141}$, V. Rossetti ${ }^{146 a, 146 b}$, E. Rossi ${ }^{104 a, 104 b}$, L. P. Rossi ${ }^{50 a}$, R. Rosten ${ }^{138}$, M. Rotaru ${ }^{26 a}$, I. Roth ${ }^{172}$, J. Rothberg ${ }^{138}$, D. Rousseau ${ }^{117}$, C. R. Royon ${ }^{136}$, A. Rozanov ${ }^{85}$, Y. Rozen ${ }^{152}$, X. Ruan ${ }^{145 c}$, F. Rubbo ${ }^{143}$, I. Rubinskiy ${ }^{42}$, V. I. Rud ${ }^{99}$, C. Rudolph ${ }^{44}$, M. S. Rudolph ${ }^{158}$, F. Rühr ${ }^{48}$, A. Ruiz-Martinez ${ }^{30}$, Z. Rurikova ${ }^{48}$, N. A. Rusakovich ${ }^{65}$, A. Ruschke ${ }^{100}$, H. L. Russell ${ }^{138}$, J. P. Rutherfoord ${ }^{7}$, N. Ruthmann ${ }^{48}$, Y. F. Ryabov ${ }^{123}$, M. Rybar ${ }^{165}$, G. Rybkin ${ }^{117}$, N. C. Ryder ${ }^{120}$, A. F. Saavedra ${ }^{150}$, G. Sabato ${ }^{107}$, S. Sacerdoti ${ }^{27}$, A. Saddique ${ }^{3}$, H. F-W. Sadrozinski ${ }^{137}$, R. Sadykov ${ }^{65}$, F. Safai Tehrani ${ }^{132 a}$, M. Saimpert ${ }^{136}$, H. Sakamoto ${ }^{155}$, Y. Sakurai ${ }^{171}$, G. Salamanna ${ }^{134 a, 134 b}$, A. Salamon ${ }^{133 a}$, M. Saleem ${ }^{113}$, D. Salek ${ }^{107}$, P. H. Sales De Bruin ${ }^{138}$, D. Salihagic ${ }^{101}$, A. Salnikov ${ }^{143}$, J. Salt ${ }^{167}$, D. Salvatore ${ }^{37 a, 37 b}$, F. Salvatore ${ }^{149}$, A. Salvucci ${ }^{106}, \quad$ A. Salzburger ${ }^{30}$, D. Sampsonidis ${ }^{154}$, A. Sanchez ${ }^{104 a, 104 b}$, J. Sánchez ${ }^{167}$, V. Sanchez Martinez ${ }^{167}$, H. Sandaker ${ }^{119}$, R. L. Sandbach ${ }^{76}$, H. G. Sander ${ }^{83}$, M. P. Sanders ${ }^{100}$, M. Sandhoff ${ }^{175}$, C. Sandoval ${ }^{162}$, R. Sandstroem ${ }^{101}$, D. P. C. Sankey ${ }^{131}$, M. Sannino ${ }^{50 a, 50 b}$, A. Sansoni ${ }^{47}$, C. Santoni ${ }^{34}$, R. Santonico ${ }^{133 a, 133 b}$, H. Santos ${ }^{126 a}$, I. Santoyo Castillo ${ }^{149}$, K. Sapp ${ }^{125}$, A. Sapronov ${ }^{65}$, J. G. Saraiva ${ }^{126 a, 126 d}$, B. Sarrazin21 ${ }^{21}$ O. Sasaki66, Y. Sasaki ${ }^{155}$, K. Sato ${ }^{160}$, G. Sauvage ${ }^{5, *}$, E. Sauvan ${ }^{5}$, G. Savage ${ }^{77}$, P. Savard ${ }^{158, d}$, C. Sawyer ${ }^{131}$, L. Sawyer ${ }^{79, n}$, J. Saxon ${ }^{31}$, C. Sbarra ${ }^{20 a}$, A. Sbrizzi ${ }^{20 a, 20 b}$, T. Scanlon ${ }^{78}$, D. A. Scannicchio ${ }^{163}$, M. Scarcella ${ }^{150}$, V. Scarfone ${ }^{37 a, 37 b}$, J. Schaarschmidt ${ }^{172}$, P. Schacht ${ }^{101}$, D. Schaefer ${ }^{30}$, R. Schaefer ${ }^{42}$, J. Schaeffer ${ }^{83}$, S. Schaepe ${ }^{21}$, S. Schaetzel ${ }^{58 b}$ ，U. Schäfer ${ }^{83}$, A. C. Schaffer ${ }^{117}$, D. Schaile ${ }^{100}$, R. D. Schamberger ${ }^{148}$, V. Scharf ${ }^{58 a}$, V. A. Schegelsky ${ }^{123}$, D. Scheirich ${ }^{129}$, M. Schernau ${ }^{163}$, C. Schiavi ${ }^{50 a, 50 b}$, C. Schillo ${ }^{48}$, M. Schioppa ${ }^{37 a, 37 b}$, S. Schlenker ${ }^{30}$, E. Schmidt ${ }^{48}$, K. Schmieden ${ }^{30}$, C. Schmitt ${ }^{83}$, S. Schmitt ${ }^{58 b}$, S. Schmitt ${ }^{42}$, B. Schneider ${ }^{159 a}$, Y. J. Schnellbach ${ }^{74}$, U. Schnoor ${ }^{44}$, L. Schoeffel ${ }^{136}$, A. Schoening ${ }^{58 b}$, B. D. Schoenrock ${ }^{90}$, E. Schopf ${ }^{21}$, A. L. S. Schorlemmer ${ }^{54}$, M. Schott ${ }^{83}$, D. Schouten ${ }^{159 a}$, J. Schovancova ${ }^{8}$, S. Schramm ${ }^{49}$, M. Schreyer ${ }^{174}, \quad$ C. Schroeder ${ }^{83}$, N. Schuh ${ }^{83}$, M. J. Schultens ${ }^{21}$, H.-C. Schultz-Coulon ${ }^{58 a}$, H. Schulz ${ }^{16}$, M. Schumacher ${ }^{48}$, B. A. Schumm ${ }^{137}$, Ph. Schune ${ }^{136}$, C. Schwanenberger ${ }^{84}$, A. Schwartzman ${ }^{143}$, T. A. Schwarz ${ }^{89}$ ， Ph. Schwegler ${ }^{101}, \quad$ H. Schweiger ${ }^{84}, \quad$ Ph. Schwemling ${ }^{136}$, R. Schwienhorst ${ }^{90}$, J. Schwindling ${ }^{136}$, T. Schwindt ${ }^{21}$, F. G. Sciacca ${ }^{17}$, E. Scifo ${ }^{117}$, G. Sciolla ${ }^{23}$, F. Scuri ${ }^{124 a, 124 b}$, F. Scutti ${ }^{21}$, J. Searcy ${ }^{89}$, G. Sedov ${ }^{42}$, E. Sedykh ${ }^{123}$, P. Seema ${ }^{21}$, S. C. Seidel ${ }^{105}$, A. Seiden ${ }^{137}$, F. Seifert ${ }^{128}$, J. M. Seixas ${ }^{24 a}$, G. Sekhniaidze ${ }^{104 a}$, K. Sekhon ${ }^{89}$, S. J. Sekula ${ }^{40}$, D. M. Seliverstov ${ }^{123, *}$, N. Semprini-Cesari ${ }^{20 a, 20 b}$, C. Serfon ${ }^{30}$, L. Serin ${ }^{117}$, L. Serkin ${ }^{164 a, 164 b}$, T. Serre ${ }^{85}$, M. Sessa ${ }^{134 a, 134 b}$, R. Seuster ${ }^{159 a}$, H. Severini ${ }^{113}$, T. Sfiligoj ${ }^{75}$, F. Sforza ${ }^{30}$, A. Sfyrla ${ }^{30}$, E. Shabalina ${ }^{54}$, M. Shamim ${ }^{116}$, L. Y. Shan ${ }^{33 a}$, R. Shang ${ }^{165}$, J. T. Shank ${ }^{22}$, M. Shapiro ${ }^{15}$, P. B. Shatalov ${ }^{97}$, K. Shaw ${ }^{164 a, 164 b}$, S. M. Shaw ${ }^{84}$, A. Shcherbakova ${ }^{146 a, 146 b}$, C. Y. Shehu ${ }^{149}$, P. Sherwood ${ }^{78}$, L. Shi ${ }^{151, a f}$, S. Shimizu ${ }^{67}$, C. O. Shimmin ${ }^{163}$, M. Shimojima ${ }^{102}$, M. Shiyakova ${ }^{65}$, A. Shmeleva ${ }^{96}$, D. Shoaleh Saadi ${ }^{95}$, M. J. Shochet ${ }^{31}$, S. Shojaii ${ }^{11 a, 91 b}$, S. Shrestha ${ }^{111}$, E. Shulga ${ }^{98}$, M. A. Shupe ${ }^{7}$, S. Shushkevich ${ }^{42}$, P. Sicho ${ }^{127}$, O. Sidiropoulou ${ }^{174}$, D. Sidorov ${ }^{114}$, A. Sidoti20a,20b ${ }^{2}$ F. Siegert ${ }^{44}$, Dj. Sijacki $^{13}$, J. Silva ${ }^{126 a, 126 d}$, Y. Silver ${ }^{153}$, S. B. Silverstein ${ }^{146 a}$, V. Simak ${ }^{128}$, O. Simard ${ }^{5}$, Lj. Simic ${ }^{13}$, S. Simion ${ }^{117}$, E. Simioni ${ }^{83}$, B. Simmons ${ }^{78}$, D. Simon ${ }^{34}$, R. Simoniello ${ }^{91 a, 91 b}$, P. Sinervo ${ }^{158}$, N. B. Sinev ${ }^{116}$, G. Siragusa ${ }^{174}$, A. N. Sisakyan ${ }^{65, *}$, S. Yu. Sivoklokov ${ }^{99}$ ， J. Sjölin 146a,146b， T. B. Sjursen ${ }^{14}$, M. B. Skinner ${ }^{72}$, H. P. Skottowe ${ }^{57}$, P. Skubic ${ }^{113}$, M. Slater ${ }^{18}$, T. Slavicek ${ }^{128}$, M. Slawinska ${ }^{107}$, K. Sliwa ${ }^{161}$, V. Smakhtin ${ }^{172}$, B. H. Smart ${ }^{46}$, L. Smestad ${ }^{14}$, S. Yu. Smirnov ${ }^{98}$, Y. Smirnov ${ }^{98}$, 
L. N. Smirnova ${ }^{99, a g}$, O. Smirnova $^{81}$, M. N. K. Smith ${ }^{35}$, R. W. Smith ${ }^{35}$, M. Smizanska ${ }^{72}$, K. Smolek ${ }^{128}$, A. A. Snesarev ${ }^{96}$, G. Snidero ${ }^{76}$, S. Snyder ${ }^{25}$, R. Sobie ${ }^{169, k}$, F. Socher ${ }^{44}$, A. Soffer ${ }^{153}$, D. A. Soh ${ }^{151, \text { af }}$, C. A. Solans ${ }^{30}$, M. Solar ${ }^{128}$, J. Solc ${ }^{128}$, E. Yu. Soldatov ${ }^{98}$, U. Soldevila ${ }^{167}$, A. A. Solodkov ${ }^{130}$, A. Soloshenko ${ }^{65}$, O. V. Solovyanov ${ }^{130}$, V. Solovyev ${ }^{123}$,P. Sommer ${ }^{48}$, H. Y. Song ${ }^{33 b}$, N. Soni ${ }^{1}$, A. Sood ${ }^{15}$, A. Sopczak ${ }^{128}$, B. Sopko ${ }^{128}$, V. Sopko ${ }^{128}$, V. Sorin ${ }^{12}$, D. Sosa ${ }^{58 b}$, M. Sosebee ${ }^{8}$, C. L. Sotiropoulou ${ }^{124 a, 124 b}$, R. Soualah ${ }^{164 a, 164 c}$, A. M. Soukharev ${ }^{109, c}$, D. South ${ }^{42}$, B. C. Sowden ${ }^{77}$, S. Spagnolo ${ }^{73 a, 73 b}$, M. Spalla ${ }^{124 a, 124 b}$, F. Spanò ${ }^{77}$, W. R. Spearman ${ }^{57}$, F. Spettel ${ }^{101}$, R. Spighi ${ }^{20 a}$, G. Spigo ${ }^{30}$, L. A. Spiller ${ }^{88}$, M. Spousta ${ }^{129}$, T. Spreitzer ${ }^{158}$, R. D. St. Denis ${ }^{53, *}$, S. Staerz ${ }^{44}$, J. Stahlman ${ }^{122}$, R. Stamen ${ }^{58 a}$, S. Stamm ${ }^{16}$, E. Stanecka ${ }^{39}$, C. Stanescu ${ }^{134 a}$, M. Stanescu-Bellu ${ }^{42}$, M. M. Stanitzki ${ }^{42}$, S. Stapnes ${ }^{119}$, E. A. Starchenko ${ }^{130}$ ，J. Stark ${ }^{55}$ ，P. Staroba ${ }^{127}$, P. Starovoitov ${ }^{42}$, R. Staszewski ${ }^{39}$ ， P. Stavina ${ }^{144 a, *}$ ， P. Steinberg ${ }^{25}$ ， B. Stelzer ${ }^{142}$ ， H. J. Stelzer ${ }^{30}$ ，O. Stelzer-Chilton ${ }^{159 a}$ ， H. Stenzel ${ }^{52}$, S. Stern ${ }^{101}$, G. A. Stewart ${ }^{53}$, J. A. Stillings ${ }^{21}$, M. C. Stockton ${ }^{87}$ ，M. Stoebe ${ }^{87}$ ，G. Stoicea ${ }^{26 a}$ ，P. Stolte ${ }^{54}$, S. Stonjek ${ }^{101}$, A. R. Stradling ${ }^{8}$, A. Straessner ${ }^{44}$, M. E. Stramaglia ${ }^{17}$, J. Strandberg ${ }^{147}$, S. Strandberg ${ }^{146,146 b}$, A. Strandlie ${ }^{119}$, E. Strauss ${ }^{143}$, M. Strauss ${ }^{113}$, P. Strizenec ${ }^{144 b}$, R. Ströhmer ${ }^{174}$, D. M. Strom ${ }^{116}$, R. Stroynowski ${ }^{40}$, A. Strubig ${ }^{106}$, S. A. Stucci ${ }^{17}$, B. Stugu ${ }^{14}$, N. A. Styles ${ }^{42}$, D. Su ${ }^{143}$, J. Su ${ }^{125}$, R. Subramaniam ${ }^{79}$, A. Succurro ${ }^{12}$, Y. Sugaya ${ }^{118}$, C. Suhr ${ }^{108}$, M. Suk ${ }^{128}$, V. V. Sulin ${ }^{96}$, S. Sultansoy ${ }^{4 c}$, T. Sumida ${ }^{68}$, S. Sun ${ }^{57}$, X. Sun ${ }^{33 a}$, J. E. Sundermann ${ }^{48}$, K. Suruliz ${ }^{149}$, G. Susinno ${ }^{37 a, 37 b}$, M. R. Sutton ${ }^{149}$, S. Suzuki ${ }^{66}$, Y. Suzuki ${ }^{66}$, M. Svatos ${ }^{127}$, S. Swedish ${ }^{168}$, M. Swiatlowski ${ }^{143}$, I. Sykora ${ }^{144 a}$, T. Sykora ${ }^{129}$, D. $\mathrm{Ta}^{90}$, C. Taccini ${ }^{134 a, 134 b}$, K. Tackmann ${ }^{42}$, J. Taenzer ${ }^{158}$, A. Taffard ${ }^{163}$ ，R. Tafirout ${ }^{159 a}$, N. Taiblum ${ }^{153}$, H. Takai ${ }^{25}$, R. Takashima ${ }^{69}$, H. Takeda ${ }^{67}$, T. Takeshita ${ }^{140}$, Y. Takubo ${ }^{66}$, M. Talby ${ }^{85}$, A. A. Talyshev ${ }^{109, c}$, J. Y. C. Tam ${ }^{174}$, K. G. Tan ${ }^{88}$, J. Tanaka ${ }^{155}$, R. Tanaka ${ }^{117}$, S. Tanaka ${ }^{66}$, B. B. Tannenwald ${ }^{111}$, N. Tannoury ${ }^{21}$, S. Tapprogge ${ }^{83}$, S. Tarem ${ }^{152}$, F. Tarrade ${ }^{29}$, G. F. Tartarelli ${ }^{91 a}$, P. Tas ${ }^{129}$, M. Tasevsky ${ }^{127}$, T. Tashiro ${ }^{68}$, E. Tassi ${ }^{37 a, 37 b}$, A. Tavares Delgado ${ }^{126 a, 126 b}$, Y. Tayalati ${ }^{135 d}$, F. E. Taylor ${ }^{94}$, G. N. Taylor ${ }^{88}$, W. Taylor ${ }^{159 b}$, F. A. Teischinger ${ }^{30}$, M. Teixeira Dias Castanheira ${ }^{76}$, P. Teixeira-Dias ${ }^{77}$, K. K. Temming ${ }^{48}$, H. Ten Kate ${ }^{30}$, P. K. Teng ${ }^{151}$, J. J. Teoh ${ }^{118}$, F. Tepel ${ }^{175}$ ， S. Terada ${ }^{66}$, K. Terashi ${ }^{155}$, J. Terron ${ }^{82}$, S. Terzo ${ }^{101}$, M. Testa ${ }^{47}$, R. J. Teuscher ${ }^{158, k}$, J. Therhaag ${ }^{21}$, T. Theveneaux-Pelzer ${ }^{34}$, J. P. Thomas ${ }^{18}$, J. Thomas-Wilsker ${ }^{77}$, E. N. Thompson ${ }^{35}$, P. D. Thompson ${ }^{18}$, R. J. Thompson ${ }^{84}$, A. S. Thompson ${ }^{53}, \quad$ L. A. Thomsen ${ }^{176}, \quad$ E. Thomson ${ }^{122}$, M. Thomson ${ }^{28}$, R. P. Thun ${ }^{89, *}$, M. J. Tibbetts ${ }^{15}$, R. E. Ticse Torres ${ }^{85}$, V. O. Tikhomirov ${ }^{96, \text { ah }, ~ Y u . ~ A . ~ T i k h o n o v ~}{ }^{109, c}$, S. Timoshenko ${ }^{98}$, E. Tiouchichine ${ }^{85}, \quad$ P. Tipton ${ }^{176}, \quad$ S. Tisserant ${ }^{85}, \quad$ T. Todorov ${ }^{5}, \quad$ S. Todorova-Nova ${ }^{129}$, J. Tojo $^{70}$,

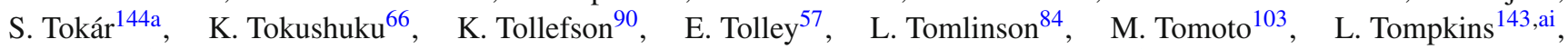
K. Toms ${ }^{105}$, E. Torrence ${ }^{116}$, H. Torres ${ }^{142}$, E. Torró Pastor ${ }^{167}$, J. Toth ${ }^{85, \text { aj }}$, F. Touchard ${ }^{85}$, D. R. Tovey ${ }^{139}$, T. Trefzger ${ }^{174}$, L. Tremblet ${ }^{30}$, A. Tricoli ${ }^{30}$, I. M. Trigger ${ }^{159 a}$, S. Trincaz-Duvoid ${ }^{80}$, M. F. Tripiana ${ }^{12}$, W. Trischuk ${ }^{158}$, B. Trocmé ${ }^{55}$, C. Troncon ${ }^{91 a}$, M. Trottier-McDonald ${ }^{15}$, M. Trovatelli ${ }^{169}$, P. True ${ }^{90}$, L. Truong ${ }^{164 a, 164 c}$, M. Trzebinski ${ }^{39}$, A. Trzupek ${ }^{39}$, C. Tsarouchas ${ }^{30}$, J. C-L. Tseng ${ }^{120}$, P. V. Tsiareshka ${ }^{92}$, D. Tsionou ${ }^{154}$, G. Tsipolitis ${ }^{10}$, N. Tsirintanis ${ }^{9}$, S. Tsiskaridze ${ }^{12}$, V. Tsiskaridze ${ }^{48}$, E. G. Tskhadadze ${ }^{51 a}$, I. I. Tsukerman ${ }^{97}$, V. Tsulaia ${ }^{15}$, S. Tsuno ${ }^{66}$, D. Tsybychev ${ }^{148}$, A. Tudorache ${ }^{26 a}$, V. Tudorache ${ }^{26 a}$, A. N. Tuna ${ }^{122}$, S. A. Tupputi20a,20b, S. Turchikhin ${ }^{99}$,ag, D. Turecek ${ }^{128}$, R. Turra ${ }^{91 a, 91 b}$, A. J. Turvey ${ }^{40}$, P. M. Tuts ${ }^{35}$, A. Tykhonov ${ }^{49}$, M. Tylmad ${ }^{146 a, 146 b}$, M. Tyndel ${ }^{131}$, I. Ueda ${ }^{155}$, R. Ueno ${ }^{29}$, M. Ughetto ${ }^{146 a, 146 b}$, M. Ugland ${ }^{14}$,

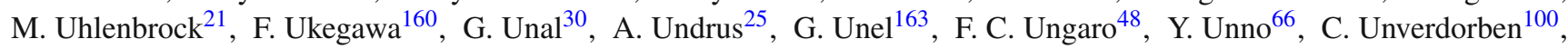

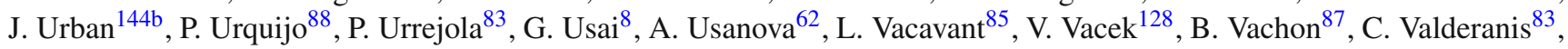
N. Valencic ${ }^{107}$, S. Valentinetti ${ }^{20 a}, 20 \mathrm{~b}$, A. Valero ${ }^{167}$, L. Valery ${ }^{12}$, S. Valkar ${ }^{129}$, E. Valladolid Gallego ${ }^{167}$, S. Vallecorsa ${ }^{49}$,

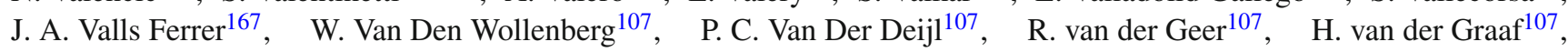
R. Van Der Leeuw ${ }^{107}$, N. van Eldik ${ }^{152}$, P. van Gemmeren ${ }^{6}$, J. Van Nieuwkoop ${ }^{142}$, I. van Vulpen ${ }^{107}$, M. C. van Woerden ${ }^{30}$, M. Vanadia ${ }^{132 a, 132 b}$, W. Vandelli ${ }^{30}$, R. Vanguri ${ }^{122}, \quad$ A. Vaniachine ${ }^{6}$, F. Vannucci ${ }^{80}, \quad$ G. Vardanyan ${ }^{177}, \quad$ R. Vari ${ }^{132 a}$, E. W. Varnes ${ }^{7}$, T. Varol $^{40}$, D. Varouchas ${ }^{80}$, A. Vartapetian ${ }^{8}$, K. E. Varvell ${ }^{150}$, V. I. Vassilakopoulos ${ }^{56}$, F. Vazeille ${ }^{34}$, T. Vazquez Schroeder ${ }^{87}$, J. Veatch ${ }^{7}$, L. M. Veloce ${ }^{158}$, F. Veloso ${ }^{126 a, 126 c}$, T. Velz ${ }^{21}$, S. Veneziano ${ }^{132 a}$, A. Ventura ${ }^{73 a, 73 b}$, D. Ventura ${ }^{86}, \quad$ M. Venturi ${ }^{169}$, N. Venturi ${ }^{158}$, A. Venturini ${ }^{23}$, V. Vercesi ${ }^{121 a}, \quad$ M. Verducci ${ }^{132 a}$,132b , W. Verkerke ${ }^{107}$, J. C. Vermeulen ${ }^{107}$, A. Vest ${ }^{44}$, M. C. Vetterli ${ }^{142, d}$, O. Viazlo ${ }^{81}$, I. Vichou ${ }^{165}$, T. Vickey ${ }^{139}$, O. E. Vickey Boeriu ${ }^{139}$, G. H. A. Viehhauser ${ }^{120}$, S. Viel ${ }^{15}$, R. Vigne ${ }^{62}$, M. Villa ${ }^{20 a, 20 b}$, M. Villaplana Perez ${ }^{91 a, 91 b}$, E. Vilucchi ${ }^{47}$, M. G. Vincter ${ }^{29}$, V. B. Vinogradov ${ }^{65}$, I. Vivarelli ${ }^{149}, \quad$ F. Vives Vaque ${ }^{3}, \quad$ S. Vlachos ${ }^{10}, \quad$ D. Vladoiu ${ }^{100}, \quad$ M. Vlasak ${ }^{128}, \quad$ M. Vogel $^{32 a}$, P. Vokac ${ }^{128}$, G. Volpi ${ }^{124 a, 124 b}$, M. Volpi ${ }^{88}$, H. von der Schmitt ${ }^{101}$, H. von Radziewski ${ }^{48}$, E. von Toerne ${ }^{21}$, V. Vorobel ${ }^{129}$, K. Vorobev ${ }^{98}, \quad$ M. $\operatorname{Vos}^{167}, \quad$ R. Voss ${ }^{30}$, J. H. Vossebeld ${ }^{74}, \quad$ N. Vranjes ${ }^{13}, \quad$ M. Vranjes Milosavljevic ${ }^{13}, \quad$ V. Vrba ${ }^{127}$, $\begin{array}{llll}\text { M. Vreeswijk } & 107\end{array}$ R. Vuillermet ${ }^{30}$, I. Vukotic ${ }^{31}, \quad$ Z. Vykydal $^{128}, \quad$ P. Wagner ${ }^{21}$, W. Wagner ${ }^{175}, \quad$ H. Wahlberg ${ }^{71}$, S. Wahrmund ${ }^{44}$, J. Wakabayashi ${ }^{103}$, J. Walder ${ }^{72}$, R. Walker ${ }^{100}$, W. Walkowiak ${ }^{141}$, C. Wang ${ }^{151}$, F. Wang ${ }^{173}$, H. Wang ${ }^{15}$, H. Wang ${ }^{40}$, J. Wang ${ }^{42}$, J. Wang ${ }^{33 a}$, K. Wang ${ }^{87}$, R. Wang ${ }^{6}$, S. M. Wang ${ }^{151}$, T. Wang ${ }^{21}$, X. Wang ${ }^{176}$, C. Wanotayaroj ${ }^{116}$, A. Warburton ${ }^{87}$, C. P. Ward ${ }^{28}$, D. R. Wardrope ${ }^{78}$, M. Warsinsky ${ }^{48}$, A. Washbrook ${ }^{46}$, C. Wasicki ${ }^{42}$, P. M. Watkins ${ }^{18}$, A. T. Watson ${ }^{18}$, I. J. Watson ${ }^{150}$, M. F. Watson ${ }^{18}$, G. Watts ${ }^{138}$, S. Watts ${ }^{84}$, B. M. Waugh ${ }^{78}$, S. Webb ${ }^{84}$, M. S. Weber ${ }^{17}$, S. W. Weber ${ }^{174}$, J. S. Webster ${ }^{31}$, A. R. Weidberg ${ }^{120}$, B. Weinert ${ }^{61}$, J. Weingarten ${ }^{54}$, C. Weiser ${ }^{48}, \quad$ H. Weits ${ }^{107}$, 
P. S. Wells ${ }^{30}$ ， T. Wenaus ${ }^{25}$ ， T. Wengler ${ }^{30}$ ， S. Wenig ${ }^{30}$, N. Wermes ${ }^{21}, \quad$ M. Werner ${ }^{48}, \quad$ P. Werner ${ }^{30}$, M. Wessels ${ }^{58 a}$, J. Wetter ${ }^{161}$, K. Whalen ${ }^{116}$, A. M. Wharton ${ }^{72}$, A. White ${ }^{8}$, M. J. White ${ }^{1}$, R. White ${ }^{32 b}$, S. White ${ }^{124 a, 124 b}$, D. Whiteson ${ }^{163}$, F. J. Wickens ${ }^{131}$, W. Wiedenmann ${ }^{173}$, M. Wielers ${ }^{131}$, P. Wienemann ${ }^{21}$, C. Wiglesworth ${ }^{36}$, L. A. M. Wiik-Fuchs ${ }^{21}$, A. Wildauer ${ }^{101}$, H. G. Wilkens ${ }^{30}$, H. H. Williams ${ }^{122}$, S. Williams ${ }^{107}$, C. Willis ${ }^{90}$, S. Willocq ${ }^{86}$, A. Wilson ${ }^{89}$, J. A. Wilson ${ }^{18}$, I. Wingerter-Seez ${ }^{5}$, F. Winklmeier ${ }^{116}$, B. T. Winter ${ }^{21}$, M. Wittgen ${ }^{143}$, J. Wittkowski ${ }^{100}$, S. J. Wollstadt ${ }^{83}$, M. W. Wolter ${ }^{39}$, H. Wolters ${ }^{126 a, 126 c}$, B. K. Wosiek ${ }^{39}$, J. Wotschack ${ }^{30}$, M. J. Woudstra ${ }^{84}$, K. W. Wozniak ${ }^{39}$, M. Wu ${ }^{55}$, M. Wu $^{31}$, S. L. Wu $^{173}$, X. Wu ${ }^{49}$, Y. Wu ${ }^{89}$, T. R. Wyatt ${ }^{84}$, B. M. Wynne ${ }^{46}$, S. Xella ${ }^{36}$, D. Xu ${ }^{33 a}$, L. Xu ${ }^{33 b, a k}$, B. Yabsley ${ }^{150}$, S. Yacoob ${ }^{145 b, a l}$, R. Yakabe ${ }^{67}$, M. Yamada ${ }^{66}$, Y. Yamaguchi ${ }^{118}$, A. Yamamoto ${ }^{66}$, S. Yamamoto ${ }^{155}$, T. Yamanaka ${ }^{155}$, K. Yamauchi ${ }^{103}$, Y. Yamazaki ${ }^{67}$, Z. Yan ${ }^{22}$, H. Yang ${ }^{33 e}$, H. Yang ${ }^{173}$, Y. Yang ${ }^{151}$, W-M. Yao ${ }^{15}$, Y. Yasu ${ }^{66}$, E. Yatsenko ${ }^{5}$, K. H. Yau Wong ${ }^{21}$, J. Ye ${ }^{40}$, S. Ye ${ }^{25}$, I. Yeletskikh ${ }^{65}$, A. L. Yen ${ }^{57}$, E. Yildirim ${ }^{42}$, K. Yorita ${ }^{171}$, R. Yoshida ${ }^{6}$, K. Yoshihara ${ }^{122}$, C. Young ${ }^{143}$,

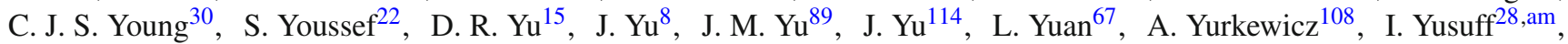
B. Zabinski ${ }^{39}$, R. Zaidan ${ }^{63}$, A. M. Zaitsev ${ }^{130, a b}$, J. Zalieckas ${ }^{14}$, A. Zaman ${ }^{148}$, S. Zambito ${ }^{57}$, L. Zanello ${ }^{132 a, 132 b}$, D. Zanzi ${ }^{88}$, C. Zeitnitz ${ }^{175}$, M. Zeman ${ }^{128}$, A. Zemla ${ }^{38 a}$, K. Zengel ${ }^{23}$, O. Zenin ${ }^{130}$, T. Ženiš ${ }^{144 a}$, D. Zerwas ${ }^{117}$, D. Zhang ${ }^{89}$, F. Zhang ${ }^{173}$, H. Zhang ${ }^{33 c}$, J. Zhang ${ }^{6}$, L. Zhang ${ }^{48}$, R. Zhang ${ }^{33 b}$, X. Zhang ${ }^{33 d}$, Z. Zhang ${ }^{117}$, X. Zhao ${ }^{40}$, Y. Zhao ${ }^{33 d, 117}$, Z. Zhao ${ }^{33 b}$, A. Zhemchugov ${ }^{65}$, J. Zhong ${ }^{120}$, B. Zhou ${ }^{89}$, C. Zhou ${ }^{45}$, L. Zhou ${ }^{35}$, L. Zhou ${ }^{40}$, N. Zhou ${ }^{163}$, C. G. Zhu ${ }^{33 d}$, H. Zhu ${ }^{33 a}$, J. Zhu ${ }^{89}$, Y. Zhu ${ }^{33 b}$, X. Zhuang ${ }^{33 a}$, K. Zhukov96, A. Zibell ${ }^{174}$, D. Zieminska ${ }^{61}$, N. I. Zimine ${ }^{65}$, C. Zimmermann ${ }^{83}$, S. Zimmermann ${ }^{48}$, Z. Zinonos ${ }^{54}$, M. Zinser ${ }^{83}$, M. Ziolkowski ${ }^{141}$, L. Živković13, G. Zobernig ${ }^{173}$, A. Zoccoli ${ }^{20 a, 20 b}$, M. zur Nedden ${ }^{16}$, G. Zurzolo ${ }^{104 a, 104 b}$, L. Zwalinski ${ }^{30}$

${ }^{1}$ Department of Physics, University of Adelaide, Adelaide, Australia

2 Physics Department, SUNY Albany, Albany, NY, USA

3 Department of Physics, University of Alberta, Edmonton, AB, Canada

4 (a) Department of Physics, Ankara University, Ankara, Turkey; ${ }^{(b)}$ Istanbul Aydin University, Istanbul,

Turkey; ${ }^{(c)}$ Division of Physics, TOBB University of Economics and Technology, Ankara, Turkey

${ }^{5}$ LAPP, CNRS/IN2P3 and Université Savoie Mont Blanc, Annecy-le-Vieux, France

${ }^{6}$ High Energy Physics Division, Argonne National Laboratory, Argonne, IL, USA

${ }^{7}$ Department of Physics, University of Arizona, Tucson, AZ, USA

${ }^{8}$ Department of Physics, The University of Texas at Arlington, Arlington, TX, USA

${ }^{9}$ Physics Department, University of Athens, Athens, Greece

10 Physics Department, National Technical University of Athens, Zografou, Greece

${ }^{11}$ Institute of Physics, Azerbaijan Academy of Sciences, Baku, Azerbaijan

12 Institut de Física d'Altes Energies and Departament de Física de la Universitat Autònoma de Barcelona, Barcelona, Spain

13 Institute of Physics, University of Belgrade, Belgrade, Serbia

14 Department for Physics and Technology, University of Bergen, Bergen, Norway

15 Physics Division, Lawrence Berkeley National Laboratory and University of California, Berkeley, CA, USA

16 Department of Physics, Humboldt University, Berlin, Germany

17 Albert Einstein Center for Fundamental Physics and Laboratory for High Energy Physics, University of Bern, Bern, Switzerland

18 School of Physics and Astronomy, University of Birmingham, Birmingham, UK

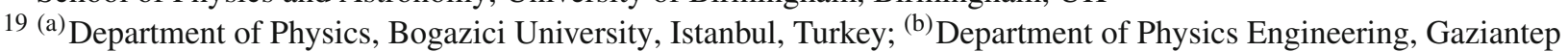
University, Gaziantep, Turkey; ${ }^{(c)}$ Department of Physics, Dogus University, Gaziantep, Turkey

20 (a) INFN Sezione di Bologna, Bologna, Italy; ${ }^{(b)}$ Dipartimento di Fisica e Astronomia, Università di Bologna, Bologna, Italy

${ }^{21}$ Physikalisches Institut, University of Bonn, Bonn, Germany

22 Department of Physics, Boston University, Boston, MA, USA

23 Department of Physics, Brandeis University, Waltham, MA, USA

24 (a) Universidade Federal do Rio De Janeiro COPPE/EE/IF, Rio de Janeiro, Brazil; (b) Electrical Circuits Department, Federal University of Juiz de Fora (UFJF), Juiz de Fora, Brazil; ${ }^{(c)}$ Federal University of Sao Joao del Rei (UFSJ), Sao Joao del Rei, Brazil; ${ }^{(d)}$ Instituto de Fisica, Universidade de Sao Paulo, São Paulo, Brazil

25 Physics Department, Brookhaven National Laboratory, Upton, NY, USA 
26 (a) National Institute of Physics and Nuclear Engineering, Bucharest, Romania; ${ }^{\text {(b) }}$ Physics Department, National Institute for Research and Development of Isotopic and Molecular Technologies, Cluj Napoca, Romania; ${ }^{(c)}$ University

Politehnica Bucharest, Bucharest, Romania; ${ }^{(d)}$ West University in Timisoara, Timisoara, Romania

${ }^{27}$ Departamento de Física, Universidad de Buenos Aires, Buenos Aires, Argentina

${ }^{28}$ Cavendish Laboratory, University of Cambridge, Cambridge, UK

${ }^{29}$ Department of Physics, Carleton University, Ottawa, ON, Canada

${ }^{30}$ CERN, Geneva, Switzerland

${ }^{31}$ Enrico Fermi Institute, University of Chicago, Chicago, IL, USA

32 (a) Departamento de Física, Pontificia Universidad Católica de Chile, Santiago, Chile; ${ }^{(b)}$ Departamento de Física, Universidad Técnica Federico Santa María, Valparaiso, Chile

33 (a) Institute of High Energy Physics, Chinese Academy of Sciences, Beijing, China; (b) Department of Modern Physics, University of Science and Technology of China, Hefei, Anhui, China; ${ }^{(c)}$ Department of Physics, Nanjing University, Nanjing, Jiangsu, China; ${ }^{(d)}$ School of Physics, Shandong University, Shandong, China; ${ }^{(e)}$ Shanghai Key Laboratory for Particle Physics and Cosmology, Department of Physics and Astronomy, Shanghai Jiao Tong University, Shanghai, China; ${ }^{(\mathfrak{f})}$ Physics Department, Tsinghua University, Beijing 100084, China

${ }^{34}$ Laboratoire de Physique Corpusculaire, Clermont Université and Université Blaise Pascal and CNRS/IN2P3, Clermont-Ferrand, France

${ }^{35}$ Nevis Laboratory, Columbia University, Irvington, NY, USA

${ }^{36}$ Niels Bohr Institute, University of Copenhagen, Copenhagen, Denmark

37 (a) INFN Gruppo Collegato di Cosenza, Laboratori Nazionali di Frascati, Frascati, Italy; ${ }^{(b)}$ Dipartimento di Fisica, Università della Calabria, Rende, Italy

38 (a) AGH University of Science and Technology, Faculty of Physics and Applied Computer Science, Kraków, Poland; ${ }^{(b)}$ Marian Smoluchowski Institute of Physics, Jagiellonian University, Kraków, Poland

${ }^{39}$ Institute of Nuclear Physics, Polish Academy of Sciences, Kraków, Poland

${ }^{40}$ Physics Department, Southern Methodist University, Dallas, TX, USA

${ }^{41}$ Physics Department, University of Texas at Dallas, Richardson, TX, USA

${ }^{42}$ DESY, Hamburg and Zeuthen, Germany

${ }^{43}$ Institut für Experimentelle Physik IV, Technische Universität Dortmund, Dortmund, Germany

${ }^{44}$ Institut für Kern- und Teilchenphysik, Technische Universität Dresden, Dresden, Germany

${ }^{45}$ Department of Physics, Duke University, Durham, NC, USA

${ }^{46}$ SUPA-School of Physics and Astronomy, University of Edinburgh, Edinburgh, UK

${ }^{47}$ INFN Laboratori Nazionali di Frascati, Frascati, Italy

${ }^{48}$ Fakultät für Mathematik und Physik, Albert-Ludwigs-Universität, Freiburg, Germany

${ }^{49}$ Section de Physique, Université de Genève, Geneva, Switzerland

50 (a) INFN Sezione di Genova, Genoa, Italy; ${ }^{(b)}$ Dipartimento di Fisica, Università di Genova, Genoa, Italy

51 (a) E. Andronikashvili Institute of Physics, Iv. Javakhishvili Tbilisi State University, Tbilisi, Georgia; ${ }^{\left({ }^{b}\right)}$ High Energy

Physics Institute, Tbilisi State University, Tbilisi, Georgia

${ }^{52}$ II Physikalisches Institut, Justus-Liebig-Universität Giessen, Giessen, Germany

${ }^{53}$ SUPA-School of Physics and Astronomy, University of Glasgow, Glasgow, UK

${ }^{54}$ II Physikalisches Institut, Georg-August-Universität, Göttingen, Germany

${ }^{55}$ Laboratoire de Physique Subatomique et de Cosmologie, Université Grenoble-Alpes, CNRS/IN2P3, Grenoble, France

${ }^{56}$ Department of Physics, Hampton University, Hampton, VA, USA

${ }^{57}$ Laboratory for Particle Physics and Cosmology, Harvard University, Cambridge, MA, USA

58 (a) Kirchhoff-Institut für Physik, Ruprecht-Karls-Universität Heidelberg, Heidelberg, Germany; ${ }^{(b)}$ Physikalisches Institut, Ruprecht-Karls-Universität Heidelberg, Heidelberg, Germany; ${ }^{(c)}$ ZITI Institut für technische Informatik, Ruprecht-Karls-Universität Heidelberg, Mannheim, Germany

${ }^{59}$ Faculty of Applied Information Science, Hiroshima Institute of Technology, Hiroshima, Japan

60 (a) Department of Physics, The Chinese University of Hong Kong, Shatin, NT, Hong Kong; ${ }^{(b)}$ Department of Physics, The University of Hong Kong, Hong Kong, Hong Kong; ${ }^{(c)}$ Department of Physics, The Hong Kong University of Science and Technology, Clear Water Bay, Kowloon, Hong Kong, China

${ }^{61}$ Department of Physics, Indiana University, Bloomington, IN, USA

${ }^{62}$ Institut für Astro- und Teilchenphysik, Leopold-Franzens-Universität, Innsbruck, Austria

${ }^{63}$ University of Iowa, Iowa City, IA, USA 
${ }^{64}$ Department of Physics and Astronomy, Iowa State University, Ames, IA, USA

65 Joint Institute for Nuclear Research, JINR Dubna, Dubna, Russia

${ }^{66}$ KEK, High Energy Accelerator Research Organization, Tsukuba, Japan

${ }^{67}$ Graduate School of Science, Kobe University, Kobe, Japan

${ }^{68}$ Faculty of Science, Kyoto University, Kyoto, Japan

${ }^{69}$ Kyoto University of Education, Kyoto, Japan

${ }^{70}$ Department of Physics, Kyushu University, Fukuoka, Japan

${ }^{71}$ Instituto de Física La Plata, Universidad Nacional de La Plata and CONICET, La Plata, Argentina

72 Physics Department, Lancaster University, Lancaster, UK

73 (a) INFN Sezione di Lecce, Lecce, Italy; ${ }^{\text {b) }}$ Dipartimento di Matematica e Fisica, Università del Salento, Lecce, Italy

74 Oliver Lodge Laboratory, University of Liverpool, Liverpool, UK

${ }^{75}$ Department of Physics, Jožef Stefan Institute and University of Ljubljana, Ljubljana, Slovenia

${ }^{76}$ School of Physics and Astronomy, Queen Mary University of London, London, UK

77 Department of Physics, Royal Holloway University of London, Surrey, UK

${ }^{78}$ Department of Physics and Astronomy, University College London, London, UK

${ }^{79}$ Louisiana Tech University, Ruston, LA, USA

${ }^{80}$ Laboratoire de Physique Nucléaire et de Hautes Energies, UPMC and Université Paris-Diderot and CNRS/IN2P3, Paris, France

${ }^{81}$ Fysiska institutionen, Lunds universitet, Lund, Sweden

82 Departamento de Fisica Teorica C-15, Universidad Autonoma de Madrid, Madrid, Spain

${ }^{83}$ Institut für Physik, Universität Mainz, Mainz, Germany

${ }^{84}$ School of Physics and Astronomy, University of Manchester, Manchester, UK

${ }^{85}$ CPPM, Aix-Marseille Université and CNRS/IN2P3, Marseille, France

86 Department of Physics, University of Massachusetts, Amherst, MA, USA

${ }^{87}$ Department of Physics, McGill University, Montreal, QC, Canada

${ }^{88}$ School of Physics, University of Melbourne, Melbourne, VIC, Australia

${ }^{89}$ Department of Physics, The University of Michigan, Ann Arbor, MI, USA

${ }^{90}$ Department of Physics and Astronomy, Michigan State University, East Lansing, MI, USA

91 (a) INFN Sezione di Milano, Milan, Italy; ${ }^{(b)}$ Dipartimento di Fisica, Università di Milano, Milan, Italy

92 B.I. Stepanov Institute of Physics, National Academy of Sciences of Belarus, Minsk, Republic of Belarus

${ }^{93}$ National Scientific and Educational Centre for Particle and High Energy Physics, Minsk, Republic of Belarus

${ }^{94}$ Department of Physics, Massachusetts Institute of Technology, Cambridge, MA, USA

${ }^{95}$ Group of Particle Physics, University of Montreal, Montreal, QC, Canada

${ }^{96}$ P.N. Lebedev Institute of Physics, Academy of Sciences, Moscow, Russia

${ }^{97}$ Institute for Theoretical and Experimental Physics (ITEP), Moscow, Russia

${ }^{98}$ National Research Nuclear University MEPhI, Moscow, Russia

99 D.V. Skobeltsyn Institute of Nuclear Physics, M.V. Lomonosov Moscow State University, Moscow, Russia

${ }^{100}$ Fakultät für Physik, Ludwig-Maximilians-Universität München, Munich, Germany

${ }^{101}$ Max-Planck-Institut für Physik (Werner-Heisenberg-Institut), Munich, Germany

102 Nagasaki Institute of Applied Science, Nagasaki, Japan

103 Graduate School of Science and Kobayashi-Maskawa Institute, Nagoya University, Nagoya, Japan

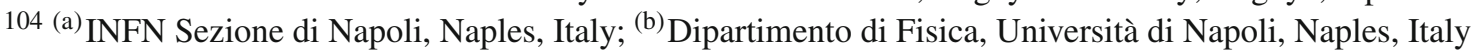

105 Department of Physics and Astronomy, University of New Mexico, Albuquerque, NM, USA

${ }^{106}$ Institute for Mathematics, Astrophysics and Particle Physics, Radboud University Nijmegen/Nikhef, Nijmegen, The Netherlands

107 Nikhef National Institute for Subatomic Physics and University of Amsterdam, Amsterdam, The Netherlands

${ }^{108}$ Department of Physics, Northern Illinois University, De Kalb, IL, USA

${ }^{109}$ Budker Institute of Nuclear Physics, SB RAS, Novosibirsk, Russia

${ }^{110}$ Department of Physics, New York University, New York, NY, USA

111 Ohio State University, Columbus, OH, USA

112 Faculty of Science, Okayama University, Okayama, Japan

${ }^{113}$ Homer L. Dodge Department of Physics and Astronomy, University of Oklahoma, Norman, OK, USA

${ }^{114}$ Department of Physics, Oklahoma State University, Stillwater, OK, USA 
115 Palacký University, RCPTM, Olomouc, Czech Republic

${ }^{116}$ Center for High Energy Physics, University of Oregon, Eugene, OR, USA

${ }^{117}$ LAL, Université Paris-Sud and CNRS/IN2P3, Orsay, France

118 Graduate School of Science, Osaka University, Osaka, Japan

${ }^{119}$ Department of Physics, University of Oslo, Oslo, Norway

${ }^{120}$ Department of Physics, Oxford University, Oxford, UK

121 (a) INFN Sezione di Pavia, Pavia, Italy; ${ }^{\text {(b) }}$ Dipartimento di Fisica, Università di Pavia, Pavia, Italy

122 Department of Physics, University of Pennsylvania, Philadelphia, PA, USA

${ }^{123}$ National Research Centre "Kurchatov Institute" B.P.Konstantinov, Petersburg Nuclear Physics Institute, St. Petersburg, Russia

124 (a) INFN Sezione di Pisa, Pisa, Italy; ${ }^{(b)}$ Dipartimento di Fisica E. Fermi, Università di Pisa, Pisa, Italy

125 Department of Physics and Astronomy, University of Pittsburgh, Pittsburgh, PA, USA

126 (a) Laboratório de Instrumentação e Física Experimental de Partículas-LIP, Lisbon, Portugal; ${ }^{(b)}$ Faculdade de Ciências, Universidade de Lisboa, Lisbon, Portugal; ${ }^{(c)}$ Department of Physics, University of Coimbra, Coimbra, Portugal; ${ }^{(\mathrm{d})}$ Centro de Física Nuclear da Universidade de Lisboa, Lisbon, Portugal; ${ }^{(\mathrm{e})}$ Departamento de Fisica, Universidade do Minho, Braga, Portugal; ${ }^{(f)}$ Departamento de Fisica Teorica y del Cosmos and CAFPE, Universidad de Granada, Granada, Spain; ${ }^{(\mathrm{g})}$ Dep Fisica and CEFITEC of Faculdade de Ciencias e Tecnologia, Universidade Nova de Lisboa, Caparica, Portugal

${ }^{127}$ Institute of Physics, Academy of Sciences of the Czech Republic, Prague, Czech Republic

${ }^{128}$ Czech Technical University in Prague, Prague, Czech Republic

${ }^{129}$ Faculty of Mathematics and Physics, Charles University in Prague, Prague, Czech Republic

${ }^{130}$ State Research Center Institute for High Energy Physics, Protvino, Russia

${ }^{131}$ Particle Physics Department, Rutherford Appleton Laboratory, Didcot, UK

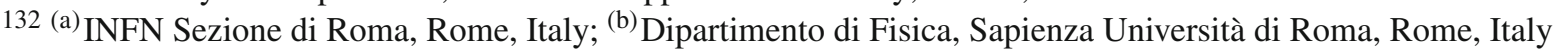

133 (a) INFN Sezione di Roma Tor Vergata, Rome, Italy; ${ }^{\left({ }^{b}\right)}$ Dipartimento di Fisica, Università di Roma Tor Vergata, Rome, Italy

134 (a) INFN Sezione di Roma Tre, Rome, Italy; ${ }^{(b)}$ Dipartimento di Matematica e Fisica, Università Roma Tre, Rome, Italy

135 (a) Faculté des Sciences Ain Chock, Réseau Universitaire de Physique des Hautes Energies-Université Hassan II, Casablanca, Morocco; ${ }^{\left({ }^{b}\right)}$ Centre National de l'Energie des Sciences Techniques Nucleaires, Rabat, Morocco; ${ }^{(c)}$ Faculté des Sciences Semlalia, Université Cadi Ayyad, LPHEA-Marrakech, Marrakech, Morocco; ${ }^{(d)}$ Faculté des Sciences, Université Mohamed Premier and LPTPM, Oujda, Morocco; ${ }^{(e)}$ Faculté des Sciences, Université Mohammed V-Agdal, Rabat, Morocco

136 DSM/IRFU (Institut de Recherches sur les Lois Fondamentales de l'Univers), CEA Saclay (Commissariat à l'Energie Atomique et aux Energies Alternatives), Gif-sur-Yvette, France

${ }^{137}$ Santa Cruz Institute for Particle Physics, University of California Santa Cruz, Santa Cruz, CA, USA

${ }^{138}$ Department of Physics, University of Washington, Seattle, WA, USA

139 Department of Physics and Astronomy, University of Sheffield, Sheffield, UK

${ }^{140}$ Department of Physics, Shinshu University, Nagano, Japan

${ }^{141}$ Fachbereich Physik, Universität Siegen, Siegen, Germany

${ }^{142}$ Department of Physics, Simon Fraser University, Burnaby, BC, Canada

${ }^{143}$ SLAC National Accelerator Laboratory, Stanford, CA, USA

144 (a) Faculty of Mathematics, Physics and Informatics, Comenius University, Bratislava, Slovak Republic; ${ }^{(b)}$ Department of Subnuclear Physics, Institute of Experimental Physics of the Slovak Academy of Sciences, Kosice, Slovak Republic

145 (a) Department of Physics, University of Cape Town, Cape Town, South Africa; ${ }^{\left({ }^{b}\right)}$ Department of Physics, University of Johannesburg, Johannesburg, South Africa; ${ }^{(c)}$ School of Physics, University of the Witwatersrand, Johannesburg, South Africa

146 (a) Department of Physics, Stockholm University, Stockholm, Sweden; ${ }^{(b)}$ The Oskar Klein Centre, Stockholm, Sweden

${ }^{147}$ Physics Department, Royal Institute of Technology, Stockholm, Sweden

${ }^{148}$ Departments of Physics and Astronomy and Chemistry, Stony Brook University, Stony Brook, NY, USA

149 Department of Physics and Astronomy, University of Sussex, Brighton, UK

${ }^{150}$ School of Physics, University of Sydney, Sydney, Australia

${ }^{151}$ Institute of Physics, Academia Sinica, Taipei, Taiwan

152 Department of Physics, Technion: Israel Institute of Technology, Haifa, Israel 
153 Raymond and Beverly Sackler School of Physics and Astronomy, Tel Aviv University, Tel Aviv, Israel

${ }^{154}$ Department of Physics, Aristotle University of Thessaloniki, Thessaloníki, Greece

${ }^{155}$ International Center for Elementary Particle Physics and Department of Physics, The University of Tokyo, Tokyo, Japan

${ }^{156}$ Graduate School of Science and Technology, Tokyo Metropolitan University, Tokyo, Japan

${ }^{157}$ Department of Physics, Tokyo Institute of Technology, Tokyo, Japan

${ }^{158}$ Department of Physics, University of Toronto, Toronto, ON, Canada

159 (a) TRIUMF, Vancouver, BC, Canada; ${ }^{(b)}$ Department of Physics and Astronomy, York University, Toronto, ON, Canada

${ }^{160}$ Faculty of Pure and Applied Sciences, University of Tsukuba, Tsukuba, Japan

${ }^{161}$ Department of Physics and Astronomy, Tufts University, Medford, MA, USA

162 Centro de Investigaciones, Universidad Antonio Narino, Bogotá, Colombia

${ }^{163}$ Department of Physics and Astronomy, University of California Irvine, Irvine, CA, USA

164 (a) INFN Gruppo Collegato di Udine, Sezione di Trieste, Udine, Italy; ${ }^{(b)}$ ICTP, Trieste, Italy; ${ }^{(c)}$ Dipartimento di Chimica Fisica e Ambiente, Università di Udine, Udine, Italy

165 Department of Physics, University of Illinois, Urbana, IL, USA

${ }^{166}$ Department of Physics and Astronomy, University of Uppsala, Uppsala, Sweden

${ }^{167}$ Instituto de Física Corpuscular (IFIC) and Departamento de Física Atómica, Molecular y Nuclear and Departamento de Ingeniería Electrónica and Instituto de Microelectrónica de Barcelona (IMB-CNM), University of Valencia and CSIC, Valencia, Spain

${ }^{168}$ Department of Physics, University of British Columbia, Vancouver, BC, Canada

${ }^{169}$ Department of Physics and Astronomy, University of Victoria, Victoria, BC, Canada

170 Department of Physics, University of Warwick, Coventry, UK

171 Waseda University, Tokyo, Japan

172 Department of Particle Physics, The Weizmann Institute of Science, Rehovot, Israel

${ }^{173}$ Department of Physics, University of Wisconsin, Madison, WI, USA

${ }^{174}$ Fakultät für Physik und Astronomie, Julius-Maximilians-Universität, Würzburg, Germany

175 Fachbereich C Physik, Bergische Universität Wuppertal, Wuppertal, Germany

${ }^{176}$ Department of Physics, Yale University, New Haven, CT, USA

177 Yerevan Physics Institute, Yerevan, Armenia

${ }^{178}$ Centre de Calcul de l'Institut National de Physique Nucléaire et de Physique des Particules (IN2P3), Villeurbanne, France

a Also at Department of Physics, King's College London, London, UK

${ }^{b}$ Also at Institute of Physics, Azerbaijan Academy of Sciences, Baku, Azerbaijan

${ }^{c}$ Also at Novosibirsk State University, Novosibirsk, Russia

d Also at TRIUMF, Vancouver, BC, Canada

${ }^{\mathrm{e}}$ Also at Department of Physics, California State University, Fresno, CA, USA

${ }^{\mathrm{f}}$ Also at Department of Physics, University of Fribourg, Fribourg, Switzerland

g Also at Departamento de Fisica e Astronomia, Faculdade de Ciencias, Universidade do Porto, Porto, Portugal

h Also at Tomsk State University, Tomsk, Russia

${ }^{i}$ Also at CPPM, Aix-Marseille Université and CNRS/IN2P3, Marseille, France

j Also at Universita di Napoli Parthenope, Naples, Italy

${ }^{k}$ Also at Institute of Particle Physics (IPP), Waterloo, Canada

${ }^{1}$ Also at Particle Physics Department, Rutherford Appleton Laboratory, Didcot, UK

${ }^{m}$ Also at Department of Physics, St. Petersburg State Polytechnical University, St. Petersburg, Russia

${ }^{n}$ Also at Louisiana Tech University, Ruston, LA, USA

${ }^{\circ}$ Also at Institucio Catalana de Recerca i Estudis Avancats, ICREA, Barcelona, Spain

p Also at Department of Physics, National Tsing Hua University, Taiwan

${ }^{q}$ Also at Department of Physics, The University of Texas at Austin, Austin, TX, USA

${ }^{\mathrm{r}}$ Also at Institute of Theoretical Physics, Ilia State University, Tbilisi, Georgia

s Also at CERN, Geneva, Switzerland

${ }^{\mathrm{t}}$ Also at Georgian Technical University (GTU), Tbilisi, Georgia

${ }^{\mathrm{u}}$ Also at Ochadai Academic Production, Ochanomizu University, Tokyo, Japan

${ }^{v}$ Also at Manhattan College, New York, NY, USA 
${ }^{w}$ Also at Hellenic Open University, Patras, Greece

${ }^{x}$ Also at Institute of Physics, Academia Sinica, Taipei, Taiwan

y Also at LAL, Université Paris-Sud and CNRS/IN2P3, Orsay, France

${ }^{\mathrm{z}}$ Also at Academia Sinica Grid Computing, Institute of Physics, Academia Sinica, Taipei, Taiwan

aa Also at School of Physics, Shandong University, Shandong, China

ab Also at Moscow Institute of Physics and Technology State University, Dolgoprudny, Russia

ac Also at Section de Physique, Université de Genève, Geneva, Switzerland

ad Also at International School for Advanced Studies (SISSA), Trieste, Italy

${ }^{\text {ae }}$ Also at Department of Physics and Astronomy, University of South Carolina, Columbia, SC, USA

af Also at School of Physics and Engineering, Sun Yat-sen University, Guangzhou, China

ag Also at Faculty of Physics, M.V.Lomonosov Moscow State University, Moscow, Russia

ah Also at National Research Nuclear University MEPhI, Moscow, Russia

ai Also at Department of Physics, Stanford University, Stanford, CA, USA

aj Also at Institute for Particle and Nuclear Physics, Wigner Research Centre for Physics, Budapest, Hungary

${ }^{\text {ak }}$ Also at Department of Physics, The University of Michigan, Ann Arbor, MI, USA

${ }^{a l}$ Also at Discipline of Physics, University of KwaZulu-Natal, Durban, South Africa

am Also at University of Malaya, Department of Physics, Kuala Lumpur, Malaysia

* Deceased 